\title{
Structure and substructure analysis of DAFT/FADA galaxy clusters in the [0.4-0.9] redshift range ${ }^{\star}$
}

\author{
L. Guennou ${ }^{1,2}$, C. Adami $^{1}$, F. Durret ${ }^{3}$, G. B. Lima Neto ${ }^{4}$, M. P. Ulmer ${ }^{5}$, D. Clowe $^{6}$, V. LeBrun ${ }^{1}$, N. Martinet ${ }^{3}$, \\ S. Allam ${ }^{7,8}$, J. Annis ${ }^{7}$, S. Basa ${ }^{1}$, C. Benoist ${ }^{9}$, A. Biviano ${ }^{3,10}$, A. Cappi ${ }^{9,11}$, E. S. Cypriano ${ }^{4}$, R. Gavazzi ${ }^{3}$, C. Halliday ${ }^{12}$, \\ O. Ilbert ${ }^{1}$, E. Jullo ${ }^{1}$, D. Just ${ }^{13,14}$, M. Limousin ${ }^{1}$, I. Márquez ${ }^{15}$, A. Mazure ${ }^{1}$, K. J. Murphy ${ }^{6}$, H. Plana ${ }^{16}$, F. Rostagni ${ }^{9}$, \\ D. Russeil ${ }^{1}$, M. Schirmer ${ }^{17,18}$, E. Slezak ${ }^{9}$, D. Tucker ${ }^{7}$, D. Zaritsky ${ }^{13}$, and B. Ziegler ${ }^{19}$
}

(Affiliations can be found after the references)

Received 31 January 2013 / Accepted 25 October 2013

\begin{abstract}
Context. The DAFT/FADA survey is based on the study of $\sim 90$ rich (masses found in the literature $>2 \times 10^{14} M_{\odot}$ ) and moderately distant clusters (redshifts $0.4<z<0.9$ ), all with HST imaging data available. This survey has two main objectives: to constrain dark energy (DE) using weak lensing tomography on galaxy clusters and to build a database (deep multi-band imaging allowing photometric redshift estimates, spectroscopic data, X-ray data) of rich distant clusters to study their properties.

Aims. We analyse the structures of all the clusters in the DAFT/FADA survey for which XMM-Newton and/or a sufficient number of galaxy redshifts in the cluster range are available, with the aim of detecting substructures and evidence for merging events. These properties are discussed in the framework of standard cold dark matter $(\Lambda \mathrm{CDM})$ cosmology.

Methods. In X-rays, we analysed the XMM-Newton data available, fit a $\beta$-model, and subtracted it to identify residuals. We used Chandra data, when available, to identify point sources. In the optical, we applied a Serna \& Gerbal (SG) analysis to clusters with at least 15 spectroscopic galaxy redshifts available in the cluster range. We discuss the substructure detection efficiencies of both methods.

Results. XMM-Newton data were available for 32 clusters, for which we derive the X-ray luminosity and a global X-ray temperature for 25 of them. For 23 clusters we were able to fit the X-ray emissivity with a $\beta$-model and subtract it to detect substructures in the X-ray gas. A dynamical analysis based on the SG method was applied to the clusters having at least 15 spectroscopic galaxy redshifts in the cluster range: $18 \mathrm{X}$-ray clusters and 11 clusters with no X-ray data. The choice of a minimum number of 15 redshifts implies that only major substructures will be detected. Ten substructures were detected both in X-rays and by the SG method. Most of the substructures detected both in X-rays and with the SG method are probably at their first cluster pericentre approach and are relatively recent infalls. We also find hints of a decreasing X-ray gas density profile core radius with redshift.

Conclusions. The percentage of mass included in substructures was found to be roughly constant with redshift values of 5-15\%, in agreement both with the general CDM framework and with the results of numerical simulations. Galaxies in substructures show the same general behaviour as regular cluster galaxies; however, in substructures, there is a deficiency of both late type and old stellar population galaxies. Late type galaxies with recent bursts of star formation seem to be missing in the substructures close to the bottom of the host cluster potential well. However, our sample would need to be increased to allow a more robust analysis.
\end{abstract}

Key words. galaxies: clusters: general

\section{Introduction}

The DAFT/FADA survey ${ }^{1}$ is based on the study of $\sim 90$ rich (masses found in the literature $>2 \times 10^{14} M_{\odot}$ ) and moderately distant $(0.4<z<0.9)$ galaxy clusters, all with HST imaging data available. This survey has two main objectives. The first one is to constrain dark energy (DE) using weak lensing tomography on galaxy clusters. The second one is to build a database of rich distant clusters to study their properties. The requirement of obtaining photometric redshifts for the DAFT/FADA survey fields has indeed allowed us to build a rich multi-band imaging database for these clusters. For a number of them, we have also obtained spectroscopic data for several tens of galaxies in the

* Tables 1,2, 4 and Appendices A-C are available in electronic form at http: //www. aanda.org

1 PIs: M. Ulmer, C. Adami, and D. Clowe, see Guennou et al. (2010) and http://cencos.oamp.fr/DAFT/ for a full description of the project. cluster redshift range, either from our own observations, or from public databases.

DAFT/FADA is a nice complement to other cluster surveys such as for example the Local Cluster Substructure Survey (LoCuSS, X-ray selected, around $z \sim 0.2$, Smith et al. 2010), the MAssive Cluster Survey (MACS, X-ray selected, $z>0.3$, but with only 12 clusters above $z=0.5$, Ebeling et al. 2001a, 2007, 2010), or the Cluster Lensing And Supernova survey with Hubble (CLASH, Postman et al. 2012) which analyses the high mass end of the cluster population, with only 14 clusters in the redshift range of our survey.

The redshift range chosen for the DAFT/FADA survey is an important one in terms of studying cluster evolution. First, because clusters have achieved nearly full growth in terms of mass by redshift of about 1 (see review by Kravtsov \& Borgani 2012, hereafter KB12, and references therein) and the DE density becomes the dominant form of energy density just below redshift 0.4 (under the standard cold dark matter, hereafter $\Lambda \mathrm{CDM}$, cosmology). Second, clusters have acquired a hot and 
dense enough intracluster medium (ICM) to become detectable at $z<2$, and yet, clusters continue to evolve, with infalling substructures present to current day, such as the Coma cluster and the NGC 4839 subgroup (Neumann et al. 2003). The [0.4, 0.9] redshift range is also interesting because it spans a time frame of about $3 \mathrm{Gyr}$, giving substructures time to infall, and thus allowing comparison of the younger systems with the older ones on a meaningful time scale. With a typical infall speed of $1000 \mathrm{~km} \mathrm{~s}^{-1}$, substructures have enough time to cross the cluster about three times between $z$ of 0.9 (age of universe $6.3 \mathrm{Gyr}$ ) and 0.4 (9.4 Gyr). Understanding how the three major components (the ICM, the galaxies, and the dark matter concentrations that are the seeds of clusters) form to grow into massive clusters is still a work in progress though (KB12).

The cluster formation simulations all involve an assumption about initial density perturbations and must include interplay between (at least) non-interacting cold dark matter (CDM) and the evolution of the baryon content in the cluster, including the ICM and galaxies.

While on the galactic scale there is a possible disagreement between the number of subhaloes found and predicted (e.g. Strigari et al. 2010), there have not been enough observations yet at the cluster and substructure scales to require any adjustments or re-examination of the $\Lambda$ CDM paradigm.

Tonnesen \& Bryan (2008) offer a useful review of cluster substructure observations. Among the numerous papers dealing with the observations of cluster substructures, we can also quote for X-rays Böhringer et al. (1994), Dupke \& Bregman (2001), Furusho et al. (2001), Shibata et al. (2001), Churazov et al. (2003), Böhringer et al. (2010), and Weissmann et al. (2013). And in the optical: Adami et al. (2005), Ulmer et al. (2009), Einasto et al. (2012), and Wen \& Han (2013). However, there has been little coupling of X-ray and optical data, especially in the redshift range $[0.4,0.9]$ of the DAFT/FADA sample. Over this time frame of about $3 \mathrm{Gyr}$, some galaxy groups are infalling for the first or second time (e.g. Poole et al. 2007 and the present paper). As noted above, clusters have already accreted enough material to become detectable by $z \sim 1$, and the time scale from $z=0.9$ to 0.4 is just sufficient for a substructure to move in and out of a cluster.

Thus, we can compare our work with the predictions of simulations such as those by Poole et al. (2007) and the observations of subgroups that are in the field. Further work done by Tonnesen \& Bryan (2008) also shows that the existence and properties of subclusters affect the evolution of ram pressure stripping of galaxies due to the local relative velocities between the substructures and the cluster galaxies. In related work on the ICM in subgroups, Takizawa (2005), for example, has shown that subclusters do not lose all their mass via ram pressure stripping, say, in the first passage, which agrees with the simulations of Poole et al. (2007). Thus, one would expect to see (as is observed) substructure in some of the cluster X-ray emission images over all redshift ranges below about 1 . In complementary work by Gao et al. (2012: the Phoenix project), they and others (e.g. Springel et al. 2008a,b; Navarro et al. 2010, the Aquarius Project) predict the fraction of mass clusters made up of subclusters (at $z=0$, however), and we can compare our results (albeit at higher redshift) with those simulations as a first step in linking these cluster formation codes with our observations.

Our data could therefore lead to improvements in the simulations to describe the substructure mass fraction growth and evolution over the $z=1-0$ time frame in a more quantitative way than results obtained, for example, by simulation movies (e.g. Diemand ${ }^{2}$ and Hydro-ski ${ }^{3}$ ) or images (e.g. Borgani \& Kravtsov (2009). Perhaps the present paper and future observational papers will encourage more quantitative simulations and their analysis.

From an observational point of view, the substructure forming groups that clusters continue to accrete at later epochs than $z \simeq 1$ have smaller scales than do the clusters themselves or those of larger groups accreted above about $z \simeq 2$ (e.g. Adami et al. 2012; Connelly et al. 2012). The existence of such groups of galaxies has been confirmed up to $z \sim 1.3$ (e.g. Gerke et al. 2007 or Lilly et al. 2009) and groups are very common at lower redshifts (e.g. Carlberg et al. 2001). This also implies different mechanisms in the group accretion (e.g. simulated in Poole et al. 2007, see also KB12) than during the initial cluster formation and should have direct consequences on the cluster dynamical state, which can be probed by detecting substructures. The search for substructures in the $[0.4,0.9]$ redshift range thus allows us to search for traces of this accretion mechanism inside galaxy clusters. This search can be made either in the galaxy distribution or in the ICM through X-ray data.

We primarily used data from the XMM-Newton archive to detect substructures in all the clusters of the DAFT/FADA survey for which such data were available (about half of the sample) and then we carried out follow-up Chandra analysis. A review of methods available to search for substructures in X-rays can be found in Andrade-Santos et al. (2012). In the optical, we used the Serna \& Gerbal (1996, hereafter SG) hierarchical code (based on spectroscopic redshifts and optical magnitudes) to detect optical substructures. A number of better-known methods are available to search for substructures in the optical, such as the $\Delta$-test (Dressler \& Schechtman 1988), which searches for deviations in the local mean velocity and velocity dispersion from the overall values. However, the SG method is quite powerful for showing evidence of substructuring, as illustrated by the results for a number of different clusters, at low redshift (Abell 496: Durret et al. 2000; Coma: Adami et al. 2005, 2009; Abell 780: Durret et al. 2009; Abell 85: Boué et al. 2008), moderate redshift (Abell 222/223: Durret et al. 2010), and high redshift (RX J1257.2+4738: Ulmer et al. 2009). The SG method has also been extensively tested on simulations by Guennou (2012), in particular on the effect of undersampling on mass determinations.

Our aim in this paper is to investigate the structure of the DAFT/FADA clusters for which X-ray and/or optical spectroscopic+imaging data are available. This will improve our knowledge of clusters (analysed in a homogeneous way) in the [0.4, $0.9]$ redshift range.

As we show below, at least in a general way, there is agreement between theory (simulations) and our observations of substructure in rich clusters, but further work is needed on both fronts to determine if the standard $\triangle \mathrm{CDM}$ model of the Universe needs any modification with regards to the effects of the CDM on the growth of large scale-structures in the Universe.

The paper is presented in the following way. In Sect. 2, we present the X-ray data, analyses, and results. Section 3 is dedicated to optical data and SG analyses. Our results are presented in Sect. 4 and summarized in Sect. 5. The majority of the figures (X-ray images and X-ray residuals over an azimuthally averaged $\beta$-model, spectroscopic redshift histograms), except for a few illustrative ones, are grouped in the Appendices (available in electronic form).

\footnotetext{
2 http://krone.physik.unizh.ch/ diemand/clusters/

3 http://astro-staff.uibk.ac.at/ hydroskiteam/
} 
We adopted the Dunkley et al. (2009) concordance cosmological model $\left(H_{0}=71.9 \mathrm{~km} \mathrm{~s}^{-1} \mathrm{Mpc}^{-1}, \Omega_{\Lambda}=0.742\right.$, $\left.\Omega_{\mathrm{M}}=0.258\right)$.

\section{X-ray data and data analysis}

We retrieved XMM-Newton data from the public archive and only kept the clusters with data of sufficient quality and depth (typically those for which the relative error on the MOS1 count rate, hence the X-ray luminosity, is less than 20\%). The XMM-Newton data obtained with the EPIC-MOS (Metal Oxide Semi-conductor) instruments were reduced using the SAS (Science Analysis System developed by the XMM-Newton team) tool from the Heasarc package. After this we applied the code created by Andy Read to remove flares, using a $3 \sigma$ clipping technique, and we calibrated the images.

\subsection{Basic parameters}

We analysed the XMM-Newton data available for 42 clusters to derive their X-ray temperatures and luminosities and search for substructures. A spatial analysis was possible for 32 of these clusters, but only 23 had deep enough X-ray data for a really robust spatial analysis (i.e. the $\beta$-model ftting process converged for those 23 clusters).

The information on the 32 clusters in our sample that have usable XMM-Newton data is given in Table 1. X-ray luminosities in the $[0.5,8.0] \mathrm{keV}$ interval were computed for all of them, but in seven cases the X-ray emission was not sufficient to estimate the temperature of the X-ray gas. For 17 clusters that we have in common with Baldi et al. (2012), we compared our X-ray gas temperatures and find good agreement (mean difference of $-0.27 \mathrm{keV}$ with a dispersion of $1.34 \mathrm{keV}$ ).

Though the main aim of this paper is to study the substructures in the DAFT/FADA survey, we computed the gas masses and total masses for the 25 clusters with measurable X-ray temperatures, and these masses can give interesting information on cluster properties. We estimated the X-ray gas masses and total masses in the $r_{500}$ radius for the clusters with reliable X-ray temperatures using the proxy calculated by Kravtsov et al. (2006), based on simulations with cosmological parameters close to ours. The parameters of the proxy determined by Kravtsov et al. (2006) are given for relaxed and unrelaxed clusters, and for $z=0$ and $z=0.6$, with small differences from one category to another. Because our X-ray clusters cover a redshift range between 0.4 and 0.9 , and some are relaxed and some are not (and in a number of cases we cannot classify our clusters as relaxed or unrelaxed), we took average values in the Kravtsov et al. (2006) Table $2\left(\log _{10} C=14.4\right.$ and $\left.\alpha=1.500\right)$ to obtain the following formulae (which give the gas and total masses in solar masses as a function of the X-ray gas temperature $k T$ in $\mathrm{keV}$ ):

$M_{\text {gas }, 500}=\frac{2.5 \times 10^{14}}{11.2}\left(\frac{k T_{\mathrm{keV}}}{3}\right)^{1.5} M_{\odot}$.

To compute the total mass, we decided to take one of the best determinants, also given by Kravtsov et al. (2006):

$Y_{x}=k T \times M_{\mathrm{gas}, 500}$.

Using the average values of the parameters (for all redshifts and all clusters: $\log _{10} C=14.27$ and $\alpha=0.581$ ), we computed the total mass with the relation:

$M_{\text {tot }, 500}=10^{14.27} \times\left(\frac{Y_{x}}{4.0 \times 10^{13}}\right)^{0.581} M_{\odot}$.
Total masses may be slightly underestimated here, since the stellar contribution (stars in galaxies and intracluster light) has not been taken into account (though it has been shown not to be negligible, see e.g. Gonzalez et al. 2007). The corresponding masses are given in Table 1. In the case of unrelaxed clusters, we may expect the total masses derived from X-rays to be overestimated (e.g. Mamon 2000; Chon et al. 2012, and references therein).

\subsection{Model subtraction to search for substructures}

The X-ray images, with a pixel size of $3.2 \times 3.2 \operatorname{arcsec}^{2}$, were then fit with an azimuthally symmetric elliptical $\beta$-model (as given by Sherpa $\left.{ }^{4}\right)$ :

$\Sigma(r)=\Sigma_{0}\left[1+\left(\frac{r}{r_{\mathrm{c}}}\right)^{2}\right]^{-3 \beta+0.5}+b$

where $\Sigma(r)$ is the surface brightness as a function of radius $r, \Sigma_{0}$ is the central surface brightness, $r_{\mathrm{c}}$ the core radius, $\beta$ the shape parameter, and $b$ accounts for the background, which is assumed to be constant throughout the image.

To analyse the best quality data possible to search for substructures, we had to make a compromise between having a high number of photons to improve our detections and avoiding artifacts due in particular to the superposition of images obtained with different detectors (and thus summing up their defects). In this context, to limit the number of artefacts, we ignored the observations that were contaminated by bad pixels and/or had CCD gaps passing through the cluster image, mainly the PN and sometimes the MOS-2 data.

We opted to model our clusters with a simple beta-model rather than with more complex ones, such as the "modified $\beta$-model" (e.g. Vikhlinin et al. 2006) or double $\beta$-model (e.g. Eckmiller et al. 2011). The reason is that many of our clusters are very faint and have a small angular size, and their cores are hardly resolved by XMM. This makes it very difficult to give meaningful constraints for models with 12 or more free parameters (remembering that we are also fitting the ellipticity, position angle, and coordinates of the centre). Therefore, to compare all clusters uniformly we used the standard 2D $\beta$-model described above.

This model represents a relaxed cluster with a homogeneous gravitational potential, simulated with the Sherpa tool ${ }^{5}$ from $\mathrm{CIAO}^{6}$. The residuals were computed as the difference between the image and the fit, allowing us to bring out any perturbation from a homogeneous gravitational potential due to the substructures that are not completely merged with the cluster yet. This is a classical technique used, for example, to study the Coma cluster with XMM-Newton (Neumann et al. 2003).

Results for each cluster are shown in the Appendix and a summary of the properties of the X-ray detected substructures is given in Table 2, together with other quantities described in Sect. 4.3. We only give the SG-estimated velocity dispersions for substructures also detected in X-rays.

\subsection{Assessing substructure detections with simulations}

To test our method of identifying substructures in X-rays, we have generated a series of synthetic X-ray images. These images

\footnotetext{
4 http://cxc.cfa.harvard.edu/sherpa4.4/ahelp/beta2d. html

5 http://cxc.harvard.edu/sherpa4.4/index.html

6 Chandra Interactive Analysis of Observations, see http://asc. harvard. edu/ciao
} 


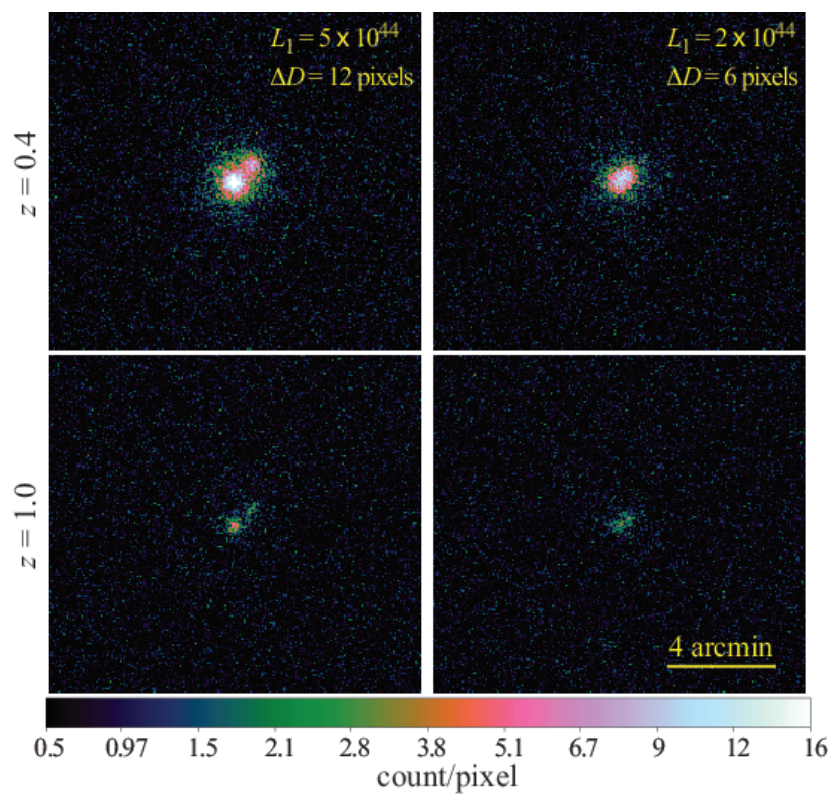

Fig. 1. A few examples of simulated images showing the situation where the subcluster can be easily detected (low redshift, well separated) and the case where the subcluster is hardly seen (high redshift, small separation). See text for more details.

consist of a primary luminous cluster and a fainter subcluster, both represented by a projected $\beta$-model, with $\beta=0.6$ (a typical value, see e.g. Jones \& Forman 1984). The surface brightness per pixel is converted in counts/s taking the MOS response, the cluster temperature, and the $K$-correction, which is important given the redshift range we are covering $(0.4 \leq z \leq 1.0)$ into account. The simulated cluster temperature is fixed using an $L_{\mathrm{X}}-T_{\mathrm{X}}$ scaling relation (Xue \& Wu 2000). The cluster images are then generated assuming a Poisson distribution for each pixel.

We assumed the equivalent of $50 \mathrm{ks}, 25 \mathrm{ks}$, and $10 \mathrm{ks}$ exposures. This optimally covers our range of exposure durations (see Table 1). The synthetic images have a scale of $3.2 \mathrm{arcsec} / \mathrm{pixel}$, corresponding to the binning we used. We added a flat Poissonian background with a count rate of $6.6 \times$ $10^{-7}$ counts $\mathrm{s}^{-1} \operatorname{arcsec}^{-2}$, corresponding to the typical on-axis MOS background in the [0.3-8.0] keV band observed in our (real) images.

We generated synthetic images in a coarse grid (see Fig. 1) where the cluster and subcluster were separated by either 6 or 12 pixels (19.2 arcsec and 38.4 arcsec, or $\sim 140$ and $280 \mathrm{kpc}$ at $z=0.7)$. For both separations, we fixed the luminosity ratio at $1 / 5$ and $1 / 2$, with the luminosity of the primary cluster fixed to be either $L_{\mathrm{X}}=5 \times 10^{44}$ or $2 \times 10^{44} \mathrm{erg} \mathrm{s}^{-1}$. Images were generated between redshifts 0.4 and 1.0 in steps of 0.1 . In this way, we generated 42 simulated images per exposure time (126 in total), to which we can apply our X-ray substructure detection procedure. The analysis of these simulations is given in Fig. 2 for $50 \mathrm{ks}$ exposures (small bumps in the curves are due to hot pixels coinciding with a substructure, which artificially increase the $\mathrm{S} / \mathrm{N})$.

We also show in Fig. 3 the detection limits of the $50 \mathrm{ks}$, $25 \mathrm{ks}$, and $10 \mathrm{ks}$ runs overlaid on our cluster distributions in a plot of useful XMM-Newton exposure time as a function of redshift. The results can be summarized as follows. There are indeed no clusters without detected substructures in Fig. 3 located inside the area where we theoretically cannot detect substructures. The detection of a substructure strongly depends on

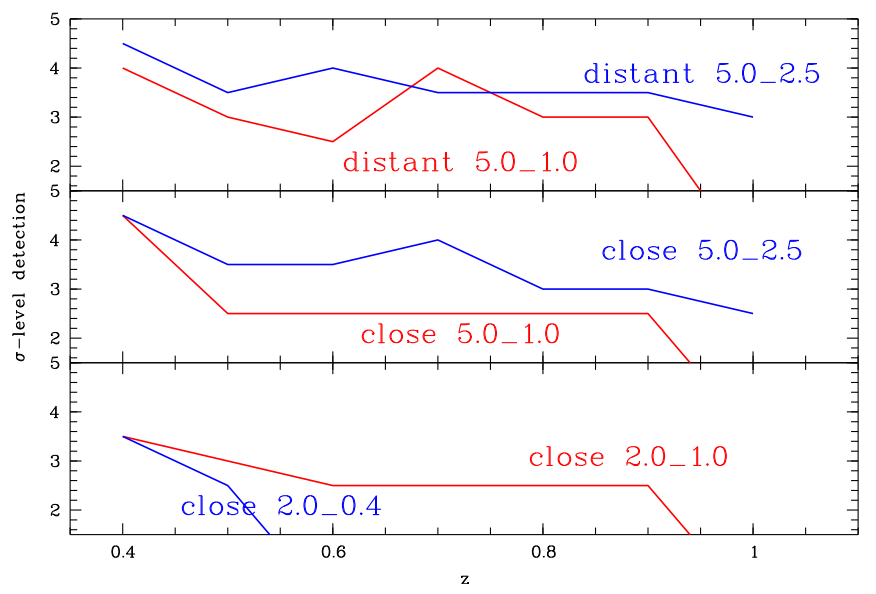

Fig. 2. Detection level (in $\sigma$ units) of the X-ray simulated substructures (50 ks exposures) versus redshift. Lower panel: $2.0 \times 10^{44} \mathrm{erg} / \mathrm{s}$ cluster + $0.4 \times 10^{44} \mathrm{erg} / \mathrm{s}$ subcluster (close 2.0_0.4) and $2.0 \times 10^{44} \mathrm{erg} / \mathrm{s}$ cluster + $1.0 \times 10^{44} \mathrm{erg} / \mathrm{s}$ subcluster (close 2.0_1.0). Separated by $6 \mathrm{px}$ in both cases. Middle panel: $5.0 \times 10^{44} \mathrm{erg} / \mathrm{s}$ cluster $+1.0 \times 10^{44} \mathrm{erg} / \mathrm{s}$ subcluster (close 5.0_1.0) and $5.0 \times 10^{44} \mathrm{erg} / \mathrm{s}$ cluster $+2.5 \times 10^{44} \mathrm{erg} / \mathrm{s} \mathrm{sub}-$ cluster (close 5.0_2.5). Separated by 6 px in both cases. Upper panel: $5.0 \times 10^{44} \mathrm{erg} / \mathrm{s}$ cluster $+1.0 \times 10^{44} \mathrm{erg} / \mathrm{s}$ subcluster (distant 5.0_1.0) and $5.0 \times 10^{44} \mathrm{erg} / \mathrm{s}$ cluster $+2.5 \times 10^{44} \mathrm{erg} / \mathrm{s}$ subcluster (distant 5.0_2.5) Separated by $12 \mathrm{px}$ in both cases. When the line stops, it means that the subcluster is no longer detected. The close 2.0_0.4 configuration, for example, provides detections of the subcluster only up to $z=0.5$ at the $2.5 \sigma$ level.

its X-ray luminosity (more than on the ratio of the luminosity of the substructure to that of the main structure). The more luminous the substructure, the better it is detected at high redshift. The distance between the substructure and the cluster centre does not change the maximum redshift of the detection, but has an influence on the significance level of the detection. It is easier to detect a substructure far from the cluster centre.

To be more quantitative, simulations predict the detection of substructures brighter than $1.0 \times 10^{44} \mathrm{erg} / \mathrm{s}$ in our redshift interval, while fainter substructures should be detected only for $z<0.5$. This is consistent with our results: all the substructures that we detect are brighter than $10^{44} \mathrm{erg} / \mathrm{s}$ except three (Abell 851, RXC J1206, and LCDCS 0504), out of which the first two clusters are at $z<0.45$. In three quarters of the cases, we also detect residuals of the cluster itself at a $2.5 \sigma$ level. Therefore, potential substructures detected very close to the cluster centre are probably only residuals from the cluster itself, and not real substructures.

The results of these simulations allow us to indicate in Table 1 whether a substructure was detected or not. If it was not detected, these simulations allow confirming that there is indeed no substructure above the solid lines in Fig. 3, or if substructures, if any, are below our detection limit (the solid lines in Fig. 3). Open symbols touching the solid lines were flagged $0 /-1$ in Table 1.

\subsection{Contamination by point sources}

In some of the residual images it was difficult to distinguish substructures from point sources owing to the limited spatial resolution of XMM-Newton. To check this point, we considered Chandra images when available. Thanks to their very good angular resolution, these data allowed us to locate bright point sources, which were usually bright enough not to be plagued by the relatively poor Chandra collecting power. 

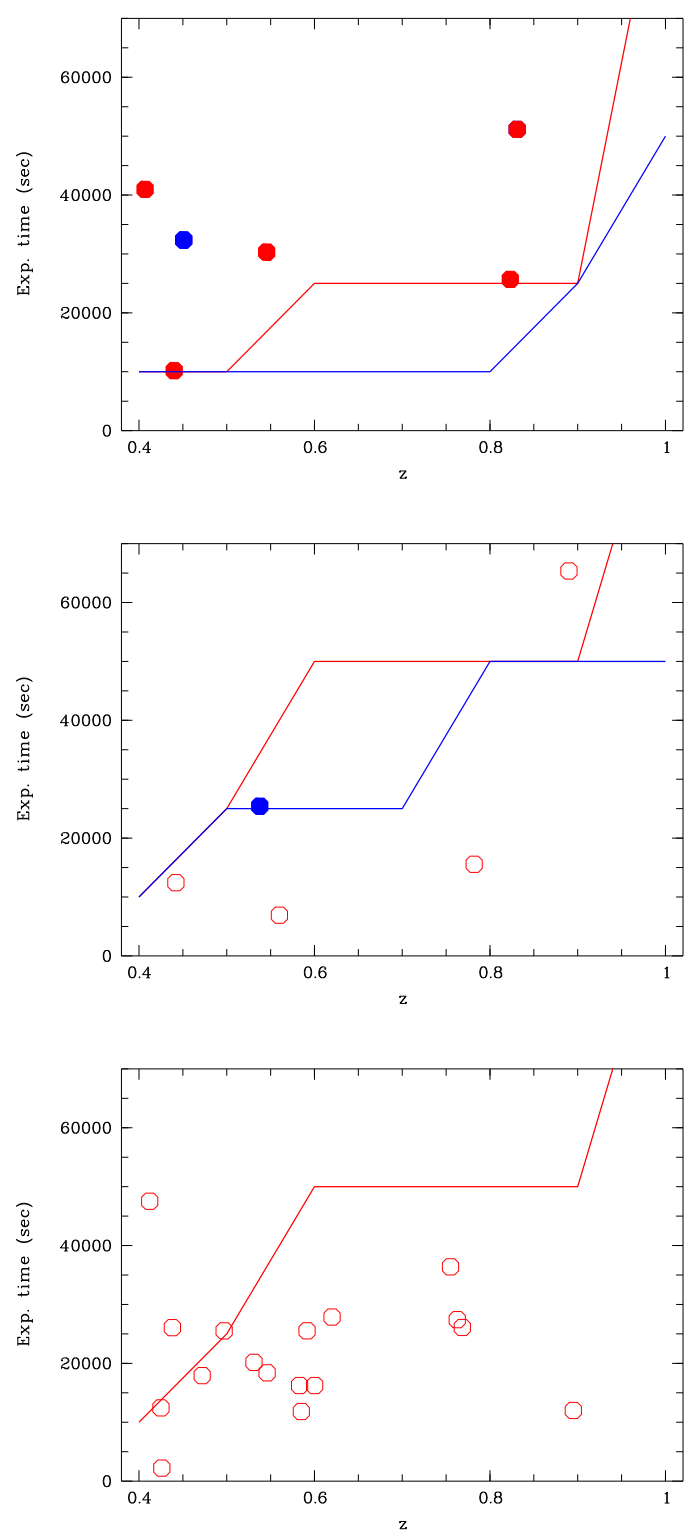

Fig. 3. Clusters in our sample in diagrams of useful XMM-Newton exposure time versus redshift. Filled symbols are clusters with one or more detected substructures, and open symbols clusters without any detected substructures. Solid lines are susbstructure detection limits below which it is not possible to detect a substructure. Upper panel: red symbols are clusters brighter than $5.0 \times 10^{44} \mathrm{erg} / \mathrm{s}$ with detected substructures fainter than $2.5 \times 10^{44} \mathrm{erg} / \mathrm{s}$ and more distant than 7 pixels from the cluster centre (or no detected substructures). Blue symbols are clusters brighter than $5.0 \times 10^{44} \mathrm{erg} / \mathrm{s}$ with detected substructures brighter than $2.5 \times 10^{44} \mathrm{erg} / \mathrm{s}$ and more distant than 7 pixels from the cluster centre. Middle panel: red symbols are clusters brighter than $5.0 \times 10^{44} \mathrm{erg} / \mathrm{s}$ with detected substructures fainter than $2.5 \times 10^{44} \mathrm{erg} / \mathrm{s}$ and less distant than 7 pixels from the cluster centre (or no detected substructures). Blue symbols are clusters brighter than $5.0 \times 10^{44} \mathrm{erg} / \mathrm{s}$ with detected substructures brighter than $2.5 \times 10^{44} \mathrm{erg} / \mathrm{s}$ and less distant than 7 pixels from the cluster centre. Lower panel: red symbols are clusters fainter than $5.0 \times 10^{44} \mathrm{erg} / \mathrm{s}$ with detected substructures fainter than $0.4 \times 10^{44} \mathrm{erg} / \mathrm{s}$ and less distant than 7 pixels from the cluster centre (or no detected substructures).

Second, we also searched for all X-ray point sources known as AGN and QSOs in the fields covered by XMM-Newton but not by Chandra. This was done via the NED and Vizier databases, as well as with our own spectroscopic observations (see below).

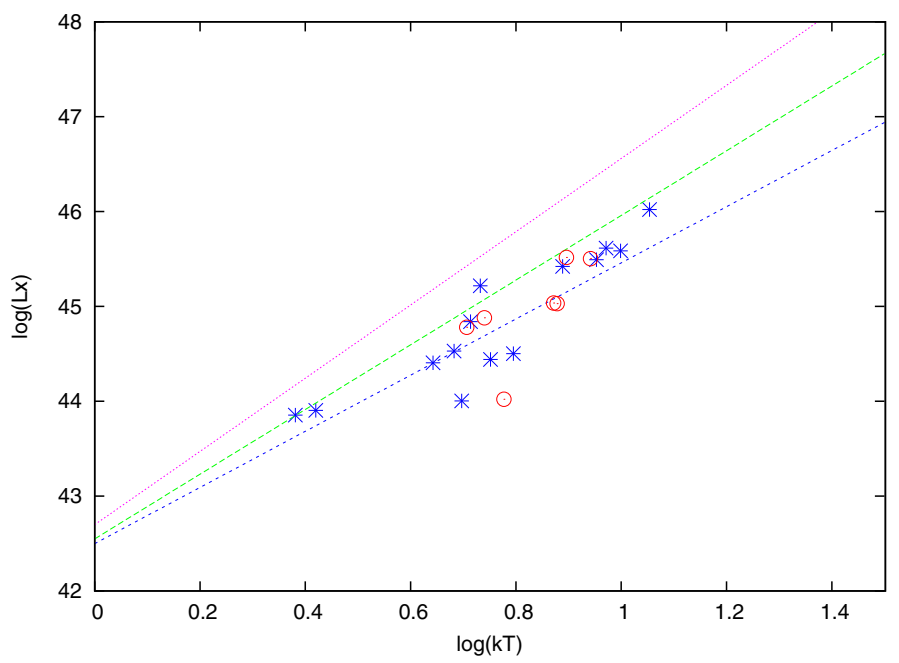

Fig. 4. X-ray luminosity as a function of X-ray temperature for our clusters on a logarithmic scale. The blue crosses and red circles represent clusters with redshifts respectively lower and higher than 0.6. The central green dashed line represents the $L_{\mathrm{X}}-T_{\mathrm{X}}$ relation found by Takey et al. (2011), and the blue and pink dashed lines indicate the uncertainty on this relation.

Most of the time, public databases referred to the work by Gilmour et al. (2009).

\subsection{Validation of $X$-ray luminosities and temperatures}

Even though this was not one of the primary goals of the present study, it was important to validate our X-ray measurements. We plot the X-ray luminosities as a function of the X-ray temperatures in Fig. 4, with different symbols for the clusters at redshifts lower and higher than 0.6 (the redshift limit of Takey et al. 2011), and we plot on the same figure the Takey et al. (2011) relation. We can see that our X-ray data agree reasonably well with the Takey et al. (2011) $L_{\mathrm{X}}-T_{\mathrm{X}}$ relation. If we try to separate clusters with and without substructures, we find no obvious dependence in the relation between $L_{\mathrm{X}}$ and $T_{\mathrm{X}}$ and the level of substructuring, perhaps because of the relatively small size of our sample.

\section{Optical data and Serna \& Gerbal analysis}

\subsection{Optical imaging and spectroscopic data}

Our survey was initiated for clusters with HST images available, generally at least in the F814W band. We obtained deep optical imaging for most of the clusters in several bands, with various telescopes (Blanco, Calar Alto, CFHT, Gemini, GTC, SOAR, TNG, VLT, WIYN, WHT). Part of the images were also taken from observatory archives (e.g. CFHT, Subaru, ESO). We are also presently in the process of acquiring infrared $J$ band images to have better constraints on the photometric redshifts of distant galaxies (for weak lensing tomography) ${ }^{7}$.

The galaxy magnitudes used here were measured in the $V$ photometric band images, calibrated and extinction-corrected in the usual way (see Guennou et al. 2010). Some archive images were observed in other bands, and in this case they were converted into the $V$ band following Fukugita et al. (1995).

We retrieved all the galaxy spectroscopic redshifts available in NED, VizieR, and in the literature in a region of radius

7 http://cencos.oamp.fr/DAFT/ 
Table 3. Clusters with no usable X-ray data but with more than 15 galaxy redshifts in the cluster range.

\begin{tabular}{lcrcc}
\hline \hline Name & RA & Dec & $z$ & $N z$ \\
\hline Cl J0023+0423B & 5.96587 & 4.37797 & 0.8453 & 23 \\
CXOMP J & 6.70917 & 17.32658 & 0.4907 & 29 \\
002650.2+171935 & & & & \\
CXOMP J & 137.86083 & 5.83681 & 0.7682 & 18 \\
091126.6+055012 & & & & \\
LCDCS 0110 & 159.46917 & -12.72889 & 0.5789 & 18 \\
LCDCS 0130 & 160.17333 & -11.93083 & 0.7043 & 30 \\
LCDCS 0172 & 163.60083 & -11.77167 & 0.6972 & 48 \\
LCDCS 0173 & 163.68125 & -12.76389 & 0.7498 & 37 \\
CL J1103.7-1245a & 165.89542 & -12.77944 & 0.6300 & 19 \\
CXOMP J & 169.35875 & 7.72639 & 0.4770 & 36 \\
111726.1+074335 & & & & \\
LCDCS 0340 & 174.54292 & -11.56639 & 0.4798 & 51 \\
LCDCS 0531 & 186.97458 & -11.63889 & 0.6355 & 24 \\
LCDCS 0541 & 188.12708 & -12.84250 & 0.5414 & 80 \\
ClG J1236+6215 & 189.16500 & 62.26500 & 0.8500 & 40 \\
XDCS mf J & 197.50792 & 32.35278 & 0.4370 & 19 \\
131001.9+322110 & & & & \\
NSCS J & 200.91500 & 30.37600 & 0.461 & 19 \\
132336+302223 & & & & \\
MJM98 034 & 203.80742 & 37.81564 & 0.383 & 16 \\
3C 295 Cluster & 212.83396 & 52.20251 & 0.4600 & 66 \\
GHO 1601+4253 & 240.80762 & 42.76005 & 0.5391 & 50 \\
RX J1716.4+6708 & 259.20667 & 67.14167 & 0.809 & 37 \\
\hline
\end{tabular}

Notes. (1) Name (as in NED), (2) right ascension in degrees (J2000.0), (3) declination in degrees (J2000.0), (4) redshift, (5) number of galaxies with redshifts in the cluster range.

5 arcmin around each cluster centre. Such a zone corresponds to a radius of $1.59 \mathrm{Mpc}$ at $z=0.4$ and $2.24 \mathrm{Mpc}$ at $z=0.8$, and therefore covers the entire cluster. We also added redshifts that we obtained during several observing runs with $8 \mathrm{~m}$ telescopes (42 redshifts obtained with GMOS on the Gemini telescope and 60 obtained with FORS 2 on the VLT). Typical errors on the velocities measured with FORS 2 are $\pm 180 \mathrm{~km} \mathrm{~s}^{-1}$ (see Guennou et al., in prep.). For redshifts taken from NED, the uncertainties are not given, so we assume them to be comparable to those of our FORS2 data.

Gemini/GMOS spectroscopy has a resolution of $7 \AA / p x$ (see Guennou et al. 2013 for details). The targeted objects were brighter than $I_{A B} \sim 24$, and the reduction was made with the IRAF package and the Gemini/Gmos environment.

VLT/FORS2 spectroscopy was obtained during ESO period 85 (programme: 085.A-0016) with a resolution of $3 \AA / \mathrm{px}$. The targeted objects were brighter than $I_{A B} \sim 23$. We applied the standard ESO reduction since it proved to be good enough to measure redshifts for most of our targets.

In order not to eliminate a priori galaxies that could be close to the clusters on the line of sight, we applied the SG method (see below) to all the galaxies with measured redshifts. For each cluster, the number of galaxies in the cluster redshift range is given in Tables 1 and 3. The cluster redshift range is defined as the range within \pm 0.025 of the mean cluster redshift (which corresponds to \pm 3 times the maximum velocity dispersion observed in clusters: $\sim 1500 \mathrm{~km} \mathrm{~s}^{-1}$ ).

Galaxy spectroscopic redshift histograms are given in the Appendix. In most cases we only show a zoom around the mean cluster redshift. When necessary, a full redshift histogram is also given (e.g. when several structures are detected along the line

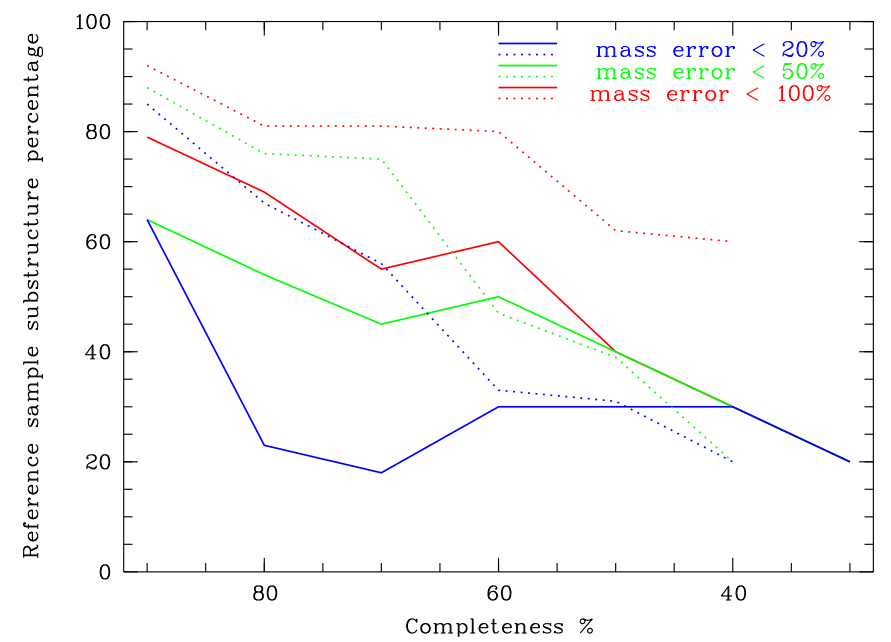

Fig. 5. Effects of the undersampling of the input spectroscopic catalogue on the results of the SG method. The figure shows the percentages of substructures in the total reference cluster sample (versus the spectroscopic catalogue completeness percentage) for which the mass estimates are plagued by an error of less than $X \%$ (compared with the $100 \%$ complete spectroscopic catalogue). Blue lines are for $X=20 \%$, green lines for $X=50 \%$, and red lines for $X=100 \%$. Continuous and dashed lines respectively correspond to substructures that merged and that did not merge with other substructures during the spectroscopic catalogue resampling process.

of sight and/or when the cluster redshift given in NED could be wrong).

\subsection{Searching for substructures by applying a Serna \& Gerbal analysis: the method}

For each cluster, the catalogue of galaxies for which positions, spectroscopic redshifts and magnitudes were available was analysed with the SG method, in a region comparable to the X-ray image (when available). We limited our sample to the clusters having at least 15 spectroscopic galaxy redshifts in the cluster range. Assuming a mean number of three SG detected substructures per cluster, this allows us to have statistically about 5 galaxies per substructure, close to the number required to avoid being too affected by incompleteness (see below).

The SG hierarchical method calculates the potential binding energy between pairs of galaxies and detects substructures taking positions and redshifts into account. The output is a file containing lists of galaxies distributed in a hierarchical way. For example, structure 1 will be the cluster and structure 2 will be galaxies outside the cluster. Structure 1 will then be divided into 11 and 12 , etc.

Assuming a value of 100 (in solar units) for the total massto-stellar luminosity ratio (but results do not strongly depend on this quantity, e.g. Adami et al. 2005), galaxy magnitudes can be transformed into masses, and approximate values can be estimated for the total masses of the various substructures detected by the SG method. The comparison with other mass estimates (see Sects. 3.4 and 4.2) strongly suggests that the absolute values of the optical masses estimated by the SG method cannot be considered as fully reliable. However, mass ratios (i.e. when trying to determine if a substructure is more massive than another one) can be considered as robust, keeping in mind, however, that assuming a constant $\mathrm{M} / \mathrm{L}$ for all galaxies is probably an oversimplification. Figure 5 allows us to estimate the typical uncertainties on these mass ratios. Considering 
only completeness levels lower than $80 \%$ (we never reach higher completeness levels in spectroscopy), the typical dispersion in mass estimates is of the order of $18 \%$. The mass ratios of two such masses therefore must have uncertainties of the order of $25 \%$ (the quadratic sum of the relative uncertainties on cluster and substructure masses, both taken to be $18 \%$ ). By $25 \%$, we mean that if a mass ratio is $40 \%$, the uncertainty on this value will be $0.25 \times 40 \%=10 \%$. Typical uncertainties on the mass ratios computed with the scaling law method (see below) are estimated to be of the same order, however we neglect here the uncertainty on the $\mathrm{M} / \mathrm{L}$ ratio assumed for the galaxies. This, by the way, makes any comparison between the stellar and total masses of the substructures difficult, in particular because by assuming the same $\mathrm{M} / \mathrm{L}$ ratio for all galaxies, mass ratios are in fact just optical luminosity ratios. Given the typical uncertainties estimated above, we chose not to give the actual values of the mass ratios in Tables 2 and 4, but rather to give estimates of these values in $10 \%$ wide intervals.

\subsection{Results of the Serna \& Gerbal analysis}

A summary of the substructures (if any) found by the SG method for each cluster is given in Table 2 .

\subsection{Influence of the undersampling of redshift catalogues}

An important question concerning the application of the SG method is that of estimating how the undersampling in the optical spectroscopy of galaxies can affect our results. To estimate the importance of this effect, we considered six reference clusters from the literature that have more than one hundred spectroscopic redshifts in the cluster redshift range and in various dynamical states (relaxed, with minor, or with major ongoing mergers). We observed the changes in the results depending on the completeness of the input catalogue. The clusters that were considered (and total numbers of galaxy redshifts in parentheses) were Abell 85 (815), Abell 168 (695), Abell 496 (499), Abell 851 (211), Abell 2744 (131), and Coma (595).

To estimate the effects of undersampling, we considered between $100 \%$ and $10 \%$ of the complete catalogue for each of the six clusters. At each step we randomly took out $10 \%$ of the galaxies, reapplied the SG, and checked the results to detect differences with growing incompleteness. This is what we call in the following the resampling process of the spectroscopic catalogue. In this way, we can observe the impact of the undersampling on substructure detection and its effects on the results (numbers of substructures found and corresponding masses) depending on the richness of the substructures.

As expected, substructures with many members tend to be detected down to lower completeness levels, while substructures with few members disappear faster when the undersampling increases. Typically, substructures detected with more than six members in the original spectroscopic catalogues will remain detected by the SG analysis down to completenesses of $\sim 50 \%$ to $30 \%$.

The precision on the mass of the substructures detected by the SG analysis varies with completeness level, as seen in Fig. 5. We distinguished two cases: (1) a given substructure is artificially merging with another one during the spectroscopic catalogue resampling process (this process will naturally tend to overestimate the substructure masses) and (2) a given substructure is not polluted by other substructures during the spectroscopic catalogue resampling process. Figure 5 shows that the
SG precision on the mass remains better than $50 \%$ for about half of the sample down to incompletenesses of about $60 \%$, while the $\mathrm{SG}$ analysis is not able to estimate the mass of most of the substructures precisely for completeness levels lower than $\sim 50 \%$.

As a further test of the influence of undersampling on our results, we also applied the SG method to a halo from the Millennium simulation (halo \#51037 100000000 ). This halo has a theoretical mass of $4.4 \times 10^{14} M_{\odot}$ and is at a redshift $z=0.37$. It has 23 subhaloes with more than three galaxies.

Considering the mass resolution of the Millennium simulation, if we use the same semi-analytical models as those applied to the CFHTLS clusters (Adami et al. 2010) we estimate that the completeness limit in this halo corresponds to an absolute magnitude $M_{I} \sim-17.5$, which roughly corresponds to the completeness limit of the DAFT/FADA survey. In this simulated cluster, if we consider $100 \%$ of the galaxies, the SG method detects the five most massive substructures (numbered from \#1 to \#5), the mass of the least massive one (\#5) being $3.5 \times 10^{12} M_{\odot}$. Two smaller structures are also detected with masses of $7.9 \times 10^{11} M_{\odot}$ and $5.2 \times 10^{11} M_{\odot}$.

If we now randomly remove $10 \%$ of the galaxies from the initial simulated galaxy catalogue, then $20 \%$, etc., we start to lose some of the initially detected substructures. The percentage of undersampling inducing the loss of a given substructure is the result of a complex interplay between the intrinsic richness of the substructure (a poor substructure will obviously be easier to lose when undersampling the catalogue) and the galaxy mass distribution in the substructure (a cD-dominated substructure will be easier to lose if the $\mathrm{cD}$ is removed). For example, because substructure \#1 is both very rich (451 galaxies) and not strongly cD-dominated (only 19\% of the total mass of the substructure is associated with the $\mathrm{cD}$ ), we are able to detect it down to a sampling rate of only $10 \%$. The other substructures disappear between sampling rates $90 \%$ and $10 \%$, but as a general statement we can say that we are able to detect some of the most massive substructures down to about $30 \%$ of the original sampling. The mass estimate remains within a factor of 2 down to $40 \%$ sampling.

We also see below that in most cases massive substructures are detected both in X-rays and with the SG analysis, when both types of data are available. However, since the SG mass estimate is sometimes inaccurate, we describe in the next section an alternative way to estimate the mass of a substructure based on optical data.

\subsection{Alternative determination of the masses of the substructures detected by the Serna \& Gerbal method}

As mentioned above, the SG algorithm allows the total structure masses to be estimated in a rather crude way, since the mass-toluminosity ratio (set to $M / L=100$ ) is assumed to be the same for all the galaxies. However, the exact value of $\mathrm{M} / \mathrm{L}$ does not strongly affect the substructure content since it mainly relies on the spectroscopic redshifts of galaxies, as shown for example by Adami et al. (2005). We have then developed a new method to estimate masses of the substructures detected with the SG algorithm based on more physical arguments. It is well known that the stellar-to-luminosity ratio of a galaxy depends on its spectrum, hence on its magnitude (e.g. Bell et al. 2003; Tremonti et al. 2004; Cappellari et al. 2006). Following this, we propose to apply a different stellar mass-to-light ratio to each galaxy, depending on its luminosity, and then to convert the stellar mass of the substructure to its total mass (i.e. including X-ray gas and 
dark matter) using the cluster scaling relation between those two quantities.

We first compute absolute magnitudes by calculating the distance modulus for each galaxy. This is made possible by our only using galaxies with an accurate spectroscopic redshift. We then convert our $V$-band magnitudes to the $g$-band, applying a colour correction of $g-V=0.28$, as found in Fukugita et al. (1995). This allows the stellar mass-to-light ratio to be estimated, as measured for SDSS data for an "average type" galaxy by Kauffmann et al. (2003, see their Fig. 14). Each galaxy is assigned its own stellar mass-to-light ratio and luminosity, taking an absolute magnitude of 5.11 for the Sun in the $g$-band. Summing the stellar masses of the galaxies belonging to the substructure provides us with the estimated stellar mass of the substructure.

The last step is to convert stellar masses to total masses using the following scaling relation defined by Giodini et al. (2009) for clusters within $\mathrm{r}_{500}$ :

$$
\frac{M_{\text {star }}}{M_{\mathrm{tot}}}=(5.0 \pm 0.1) \times 10^{-2}\left(\frac{M_{\mathrm{tot}}}{5 \times 10^{13} M_{\odot}}\right)^{-0.37 \pm 0.04} \text {. }
$$

One must note that the \pm 0.04 uncertainty on the exponent in the previous equation results in large error bars on the total substructure mass (about $85 \%$ ).

We calculated substructure masses following this method and compared them to those calculated with the SG method for the 18 clusters with optical substructures and X-ray data (e.g. Table 2) and we have a rough overall agreement. We are aware that none of these methods gives the exact mass of substructures, so we present both to cross check our mass ratios. When the results obtained with both methods agree, we have a good chance of having a reasonable estimate.

We also calculated substructure masses for the clusters to which we applied the SG method but for which no X-ray analysis was possible, and give results in Table 4.

\section{X-ray gas distribution}

The results for the invidual clusters with X-rays and/or a SG analysis are described in the Appendix. In Appendix A, we give a full X-ray (and optical when enough galaxy redshifts are available) analysis for the clusters with usable XMM-Newton data. In Appendix B, we present the SG analysis for the clusters with no usable X-ray data but with at least 15 galaxy redshifts in the cluster range. In Appendix $\mathrm{C}$ we give brief notes on the clusters for which we have little spatial information, but which are worth mentioning in particular because several of them seem to have redshifts differing from those given by NED.

\subsection{Cluster core radius versus redshift}

We first investigate the possible variations in the cluster $\mathrm{X}$-ray gas distribution (modelled by a $\beta$-model) versus redshift. There is no significant correlation between the redshift and the $\beta$-model slope. We may, however, detect a tendency between the $\beta$-model core radius and the redshift (see Fig. 6). To produce this figure, we first only selected clusters for which the $\beta$-model fitting provides a converging solution (true for $\sim 80 \%$ of the clusters). Then, we eliminated double clusters as CL J0152.7-1357. Figure 6 shows that clusters at redshifts lower than 0.65 tend to have larger core radii.

If we now eliminate the clusters with X-ray-detected substructures for which the core radius may be biased the tendency



Fig. 6. $\beta$-model core radius as a function of redshift for the considered clusters (see text). The circled disks are the clusters with detected X-ray substructures. The two lines symbolize the $z=0.65$ limit and the maximal value of the core radii for $z \geq 0.65$ not significantly substructured clusters (from an X-ray point of view).

is even more pronounced. The respective mean core radii are $76 \pm 42 \mathrm{kpc}, 93 \pm 45 \mathrm{kpc}$, and $34 \pm 13 \mathrm{kpc}$ for the clusters in the redshift bins $[0.4 ; 0.5],[0.5 ; 0.65]$, and $[0.65 ; 0.9]$. Even though our sample is limited (only 16 clusters in total after all the selections), it suggests that the highest redshift clusters are younger structures that have not yet accreted large amounts of matter and therefore have smaller core radii. However, this could be due to several selection effects, since our cluster sample is not homogeneous in terms of numbers of detected clusters versus redshift: it is a collection of clusters known in the literature to which we applied simple criteria on mass and available data (see Sect. 1).

The first question is to know if the XMM-Newton data we have in hand are able to measure large core radii at $z \geq 0.65$. The collected XMM-Newton data are of two types: (1) pointed observations for which the cluster was the main target or (2) observations made for other purposes and where clusters were observed by chance.

We can hope that type (1) XMM-Newton pointings will not be too affected by the inability to measure core radii, because exposure times have been selected by the original PIs to specifically study the clusters and modelling a gas distribution is one of the most basic tasks. To test this point, we computed that $\sim 80 \%$ of core radius measurements in the type (1) pointings were successful. The calculation of the core radius with Sherpa converged for $80 \%$ of the clusters. Among the $\sim 20 \%$ unsuccessful measurements, half were at $z \geq 0.65$ and half at $z \leq 0.65$. Type (1) observations therefore do not seem to be affected by a variable (with redshift) ability to measure core radii. Similarly, we also checked that $z \geq 0.65$ and $z \leq 0.65$ did not provide X-ray photon count rates that are too different (the basic parameter for modelling the gas distribution). Clusters at $z \leq 0.65$ are sometimes very bright, but if these bright objects are excluded, we have somewhat lower count rates at low redshifts than at high reshifts. Almost $40 \%$ of the $z \leq 0.65$ clusters have count rates that are higher than 0.04 counts/s, while $50 \%$ of the $z \geq 0.65$ clusters have count rates higher than 0.04 counts/s. We therefore do not find a strong variation in the cluster count rates with redshift in our sample.

Type (2) observations could present a variable core radius measurement efficiency because their exposure times were not specifically selected to study the clusters. However, these 




Fig. 7. Cluster total X-ray luminosity as a function of redshift. Filled disks are clusters for which substructures were detected with the X-ray method. Crosses indicate clusters with no X-ray detected substructures. The blue and red circles correspond to clusters for which substructures representing less and more respectively than $15 \%$ of the total cluster mass (estimated with the SG method) were detected with the SG method.

observations represent only $17 \%$ of the sample, and only one third of them corresponds to $z \geq 0.65$ clusters. The effect is therefore limited.

A second bias could be due to the fact that X-ray clusters of galaxies often exhibit relations between size, luminosity, and temperature (the well known fundamental plane of galaxy clusters, e.g. Adami et al. 1998). Since distant clusters in the literature are more easily detected when they are luminous (and therefore have high luminosity or temperature), we may imagine a tendency to select clusters with small core radii due to the selection of high-luminosity or high-temperature clusters. To test this point, we checked that relations between the core radius and the luminosity or temperature were visible in our sample. We did not detect any clear correlations, so even a luminosity or temperature selection effect in our sample would not induce a core radius effect, such as the one seen in Fig. 6.

However, that the high redshift clusters could systematically have smaller core radii will have to be confirmed on larger samples in the coming years. We detect no correlation between the core radius and the X-ray temperature (and therefore the X-ray mass), and we detect no clear relation between the velocity dispersion and the X-ray luminosity either.

\subsection{X-ray versus SG substructures}

Figure 7 shows that substructures were detected with the X-ray method at all redshifts. However, it is mainly the most X-ray luminous clusters $\left(\geq 4 \times 10^{44} \mathrm{erg} / \mathrm{s}\right)$ that provided such detections. To check that these are instrumental effects (only luminous clusters could provide substructure detections due to their higher $\mathrm{S} / \mathrm{N}$ ), we put the substructure information coming from the SG analysis on the same figure. We see that we detected substructures with the SG method even for clusters with undetected $\mathrm{X}$-ray substructures, and even for clusters with low X-ray luminosities. Therefore, X-ray selection effects must indeed be at work. However, nearly all the substructures undetected in X-rays but detected by the SG method seem to be minor (less than $15 \%$ of the total cluster mass). It is therefore tempting to conclude

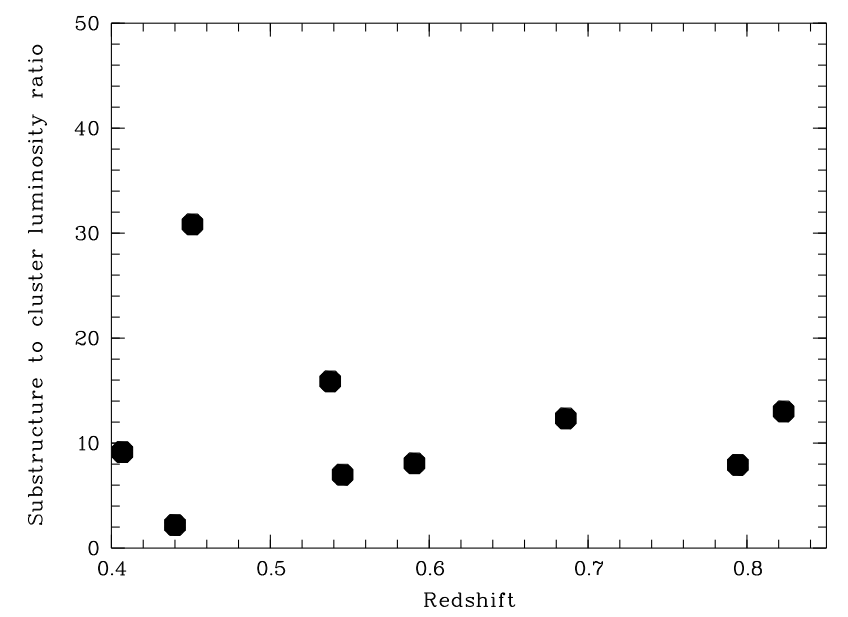

Fig. 8. Substructure to cluster luminosity ratios from the X-ray analysis (in \%) versus redshift.

that all the major substructures (or at least a large percentage of these) were effectively detected with the X-ray data in hand.

We can now examine the variations in the X-ray luminosity ratio (substructure versus cluster) as a function of redshift. We find that this percentage remains more or less constant with redshift in a 5-15\% interval (see Fig. 8). Even if such percentages are probably an underestimate of the mass fraction of clusters that is in the form of substructures (because we do not detect them all, see Sect. 2.3), they are in good agreement with the Millennium simulation (see Appendix of Adami et al. 2013 or Gao et al. 2012) predicting that clusters below $z \sim 1$ primarily undergo minor mergers.

\subsection{X-ray substructure merging stages}

We concentrate in this part on substructures detected both in $\mathrm{X}$-rays and with the SG method. We consider the X-ray substructures that we detected as relics of more or less recently infalling groups of galaxies. We were able to measure physical parameters for these groups based on their X-ray gas phase and on their galaxy phase. Gas and galaxies have different time-scales in galaxy structures in response to gravitational perturbations such as mergers. We are therefore theoretically in the position of deducing the merging stage of the considered infalling groups with the corresponding clusters.

More precisely, we know the position of isolated groups in $L_{\mathrm{X}}$ versus galaxy velocity dispersion diagrams (e.g. Connelly et al. 2012). We compared the substructures that we have detected in the present paper to these isolated groups. This was done after applying a 1.73 correction factor to translate the Connelly et al. (2012) X-ray luminosities (measured in the $0.1-2.4 \mathrm{keV}$ energy range (see their Sect. 3.1 for details) to our $0.5-8 \mathrm{keV}$ energy range.

Four of our substructures exhibit velocity dispersions over $500 \mathrm{~km} \mathrm{~s}^{-1}$. This may appear large for dynamically relaxed groups. For SG1 in CL J0152.7-1357, it is not surprising because this cluster is undergoing a major merger, so SG1 is already a pretty massive structure. It is more difficult to explain the high values for Abell 851 (SG3), MS 1054-03 (SG1), and RXC J1206.2-0848 (SG4) if they are relaxed. We may then deal with highly unrelaxed groups in an already quite advanced fusion stage.

We clearly see in Fig. 9 that most of our substructures have higher X-ray luminosities than the isolated groups of Connelly 




Fig. 9. X-ray luminosity as a function of galaxy velocity dispersion. Open circles are from Connelly et al. (2012), red: X-ray selected groups, blue: optically selected groups. The two black curves show the $3 \sigma$ envelope of the Connelly et al. (2012) X-ray selected sample. The black filled circles correspond to our detected substructures. The lower extremities of the vertical lines show the places of the infalling structures prior to their dynamical capture assuming the epoch written next to the lines. Other epochs written in parentheses are also possible to place the infalling structures inside the Connelly et al. (2012) envelope.

et al. (2012). This shows that, as expected, our substructures are not classical isolated groups but have already undergone some transformations when falling into the clusters.

Merging simulations (e.g. Poole et al. 2007) show that these transformations include, among others, several increases in the main cluster X-ray luminosity during the merging process at well-defined epochs. More precisely, there is a first increase of the X-ray luminosity at the epoch that we will call $t 0$ in the present paper (first virial encounter of the infalling structure with the impacted cluster), then a more significant one at $t 1$ (first pericentre approach of the infalling structure), and a third and a fourth smaller increases at epochs $t 2$ (second pericentre approach) and $t 3$ (relaxation of the impacted structure).

That we still detect X-ray substructures in our clusters naturally excludes the $t 3$ epoch. Given the depth of our X-ray data, we never detect the cluster X-ray contributions up to the virial radius, and this prevents us from detecting substructures at the $t 0$ stage. Our clusters are therefore somewhere between the $t 1$ and $t 2$ epochs.

The simulations of Poole et al. (2007) quantified the increase in the X-ray luminosity with time. By applying their predicted increasing factors for major mergers (which the infalling groups experience when merging with a larger cluster) and considering the $t 1$ and $t 2$ epochs, we were able to estimate the original $\mathrm{X}$-ray luminosities of our infalling groups prior to their gravitational capture. We then selected the possible epochs $(t 1$ and/or $t 2$ ), allowing the infalling groups to have been optimally located in the $L_{\mathrm{X}}$ versus velocity dispersion diagram for isolated groups (from Connelly et al. 2012) before gravitational capture. These epochs are listed in Table 2. The increasing factors that we applied are applicable to clusters merging with another cluster of comparable mass. This is not the case here, but it is the best estimation at our disposal of the increase in X-ray luminosity caused by a merger. Poole et al. (2007) did not simulate what happens for merging ratios greater than 1 . The $\mathrm{X}$-ray luminosity shifts in Fig. 9 could therefore be greater. We see that most of the X-ray detected substructures are probably at the $t 1$ epoch (first pericentre approach) and therefore are relatively recent in the clusters.

\section{Galaxy content in substructures}

As already mentioned, we collected ground-based imaging and spectroscopy at visible and near-infrared wavelengths. Our ultimate goal is to gather at least five bands in the visible domain (typically $\left.B, V, R, I, z^{\prime}\right)$ and one in the near-infrared domain ( $J$ or $K \mathrm{~s}$ ) to compute photometric redshifts with the LePhare tool (see Guennou et al. 2010). We are in the process of completing this data collection for our $\sim 90$ lines of sight, but for now all clusters of Table 2 with detected substructures (except MS 1621.5+2640) have the full dataset available. This allowed us to compute photometric redshifts for these clusters, and we are therefore now ready to investigate the galaxy content of these structures and of their detected substructures.

The LePhare tool, as for other photometric redshift tools, can be used to compute photometric redshifts, as well as to characterize the considered galaxies in terms of type or stellar population age (e.g. Adami et al. 2009). If we can fix the redshift at its true value (considering only spectroscopic catalogues), then we limit the number of free parameters and we have even better constraints on the type and stellar population ages. This is the approach we have presently chosen, with the spectroscopic catalogues at our disposal. We selected galaxies with a known spectroscopic redshift and chose Bruzual \& Charlot (2003) spectral templates in LePhare, fixed the redshift to its value, and estimated the stellar population ages and photometric types. These photometric types are arbitrarily coded between 10 (early galaxies) and 31 (late starbursts). We then investigated the galaxy distribution in an age versus type space. We assume that the age can be considered as driven by the epoch of the last burst of star formation.

\subsection{Cluster versus field galaxies}

The first thing to check is the general behaviour of cluster versus field galaxies. In order to limit the contamination of the cluster sample by field galaxies as much as possible, we defined the cluster sample as all galaxies with a redshift differing by less than 0.01 from the lower redshift and higher redshift substructures in Table 2. This corresponds to three times the typical velocity dispersion of a massive cluster. The field sample was defined as all galaxies with a redshift differing by more than 0.02 from the lower redshift and higher redshift subtructures in Table 2. This corresponds to six times the typical velocity dispersion of a massive cluster. We are aware that this eliminates galaxies between three and six times the typical cluster velocity dispersion, but these galaxies are potentially in an intermediate state, and they would have made our results noisier.

Figure 10 shows the expected behaviour in both the field and cluster samples, with a population of earlier type galaxies more prominent in the cluster sample. We see a clear dichotomy between early and late types happening around type 17 for both the cluster and the field samples, the gap being less populated in the cluster sample.

Performing a two-dimensional Kolmogorov-Smirnov test gives a probability greater than $99.9 \%$ to have populations (cluster and field galaxies) coming from different parent samples. This behaviour has been reported before, but it confirms that our approach to compute age and type is valid. 


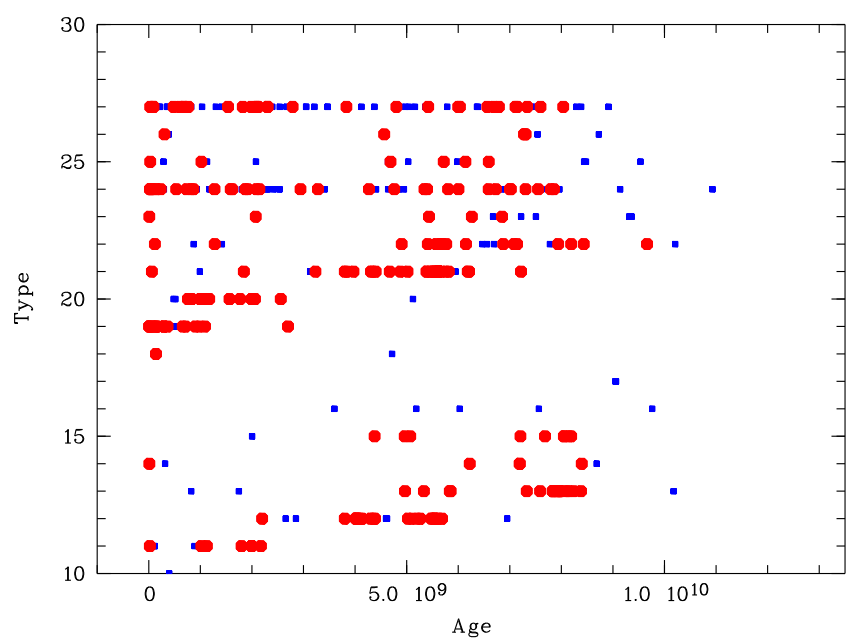

Fig. 10. Cluster (large red circles) and field (small blue squares) galaxies in an age versus type plot.



Fig. 11. Galaxies in SG detected (small green disks) and SG+X-rays detected (large black disks) substructures in a plot of galaxy type versus age.

\subsection{General position of galaxies in substructures}

We first note that galaxies members of substructures are very different from field galaxies. A Kolmogorov-Smirnov test gives a probability over $99.9 \%$ to have populations (galaxies in substructures and field galaxies) coming from different parent samples. Figure 11 shows that there is perhaps a lack of both late-type and old stellar population galaxies in substructures detectable in X-rays. This could be explained if galaxies in $\mathrm{X}$-ray detected substructures had undergone recent bursts of star formation induced by shocks in the hot medium. However, this has to be confirmed on larger samples.

\subsection{Galaxies in substructures as a function of substructure characteristics}

Given the modest size of the galaxy samples in substructures detected both by the SG and X-ray methods, we concentrate here on the substructures detected at least by the SG method in order to maximize our sample.

We chose to characterize the substructures by their velocity difference with the mean cluster velocity. This gives an idea of the importance of the cluster influence on the substructure.

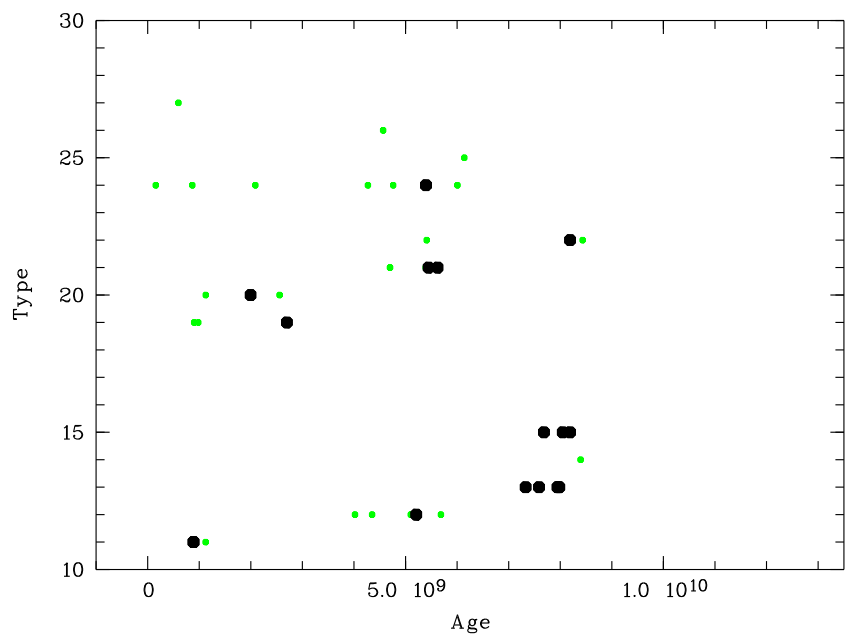

Fig. 12. Galaxies in SG detected substructures at less than $900 \mathrm{~km} \mathrm{~s}^{-1}$ (small green disks) and at less than $300 \mathrm{~km} \mathrm{~s}^{-1}$ (large black disks) from the mean cluster velocity in a plot of galaxy type versus age.

For example, the difference between the substructure members closer than $300 \mathrm{~km} \mathrm{~s}^{-1}$ and $900 \mathrm{~km} \mathrm{~s}^{-1}$ from the cluster mean velocity appears in Fig. 12 to be due to the lack of late type galaxies with recent bursts of star formation in the substructures closest to the cluster mean velocity. This may indicate that substructures close to the bottom of the cluster potential well have consumed a large part of their gas and are therefore less able to form new generations of stars.

\subsection{Summary}

In conclusion to this section, we can say that galaxy populations in substructures have the same general behaviour as regular cluster galaxies, but with several noticeable differences:

- a possible lack of both late type and old stellar population galaxies in substructures detectable in X-rays and in SG substructures contributing the most to the cluster mass,

- a possible lack of late type galaxies with recent bursts of star formation in the closest substructures to the cluster mean velocity.

These tendencies can be explained by classical expected behaviours in clusters where galaxies close to the bottom of the cluster potential have probably consumed a large part of their gas and are therefore less able to form new generations of stars, and where galaxies in important substructures would have undergone recent bursts of star formation initiated by shocks induced in the hot medium and energy transfer from the surrounding cluster.

\section{Discussion}

We studied a sample of 32 clusters of galaxies with usable X-ray data and of 19 clusters of galaxies without X-ray data but with more than 15 available spectroscopic redshifts in the cluster range. Ten substructures were detected both in X-rays and by the SG method at optical wavelengths.

We eliminated point source contamination by using Chandra data when available and with public catalogues of active galactic nuclei or radio sources. We detected substructures based on X-ray analysis via the statistically significant detection of residuals based on a surface-brightness $\beta$-model subtraction, or 
optically via an application of the SG dynamical method to our spectroscopic redshift catalogues.

From this work we derived a new set of substructures in rich clusters of galaxies in the redshift range $[0.4,0.9]$. We have verified that these are dynamically bound systems by combining the detection in X-rays with a dynamical analysis based on spectroscopic redshifts. We now discuss these results in the context of cluster evolution, as well as in comparison with previous work, where appropriate. A major goal in understanding cluster formation is to be able to use clusters as a tool for studying cosmology, we begin with a brief summary of the relationship between clusters and cosmology.

Because astrophysicists have come to realize there is a direct link between (1) clusters, (2) how large scale structure formed, (3) dark matter, and (4) dark energy, clusters of galaxies are being used more and more as cosmological tools (see for example Allen et al. 2011 and Kravtsov \& Borgani 2012). Those works (and references therein) show how cluster counts and clustercluster correlations may shed light on the Gaussianity or nonGaussianity of the initial primordial fluctuations in the early Universe. Furthermore, these papers and others have shown that studies of clusters can also be used to delimit the value of $w$, if $w$ is variable with redshift, or if there are deviations from General Relativity that are causing the apparent acceleration of the Universe (e.g. Vikhlinin et al. 2009; Allen et al. 2011; Kravtsov \& Borgani 2012). On the finest cosmological scale of galaxies, work is going on to determine if there is indeed a missing sub-halo problem or not, but so far there is no consensus (see for example Strigari et al. 2010).

On the in-between scales of groups of galaxies that are continually falling into galaxy clusters, the hierarchical build up of clusters with groups has been described both in published works, such as Poole et al. (2007), Gao et et al. (2012, and references therein, too many to list them all), and the Millennium project (Springel et al. 2005), but also in simulations posted on the web.

The Poole et al. (2007) results are in partial agreement with our data, as are those of Gao et al. (2012), in that (a) we find that the X-ray detected groups are in the luminous stage as if they were "lit up" by infall to the cluster as predicted by Poole et al. (2007); (b) Gao et al. (2012) found that at redshift $z=0$, the total masses in substructures relative to the total cluster masses in their simulations were about $5-15 \%$. This $5-15 \%$ value is about the same as the one we found for clusters in our sample, but what is uncertain is how this percentage should change (if at all) with redshift between 0.4 and 0.9. For example, a cluster initially without substructure could grow in such a way that at higher redshifts its initial mass is relatively low, so that added sub-clumps are a relatively high percentage of the total cluster mass. Conversely, it could be that as clusters grow, many of the substructures are not dissipated enough to disappear, and the total mass in substructures actually grows over time. That our mass percentage in substructures at $z=0.4-0.9$ is about equal to what is predicted by Poole et al. (2007) at $z=0$ argues that most likely events conspire to keep the detectable mass in substructures in clusters approximately constant from $z=0.9$ to the current day. This would also be in good agreement with the quite constant level of diffuse light present in clusters between $z=0.4$ and 0.9 (see Guennou et al. 2012).

The work of Mann \& Ebeling (2012) states that the fraction of "disturbed" clusters increases with increasing redshift, implying a higher substructure mass with higher redshift, in apparent contradiction to our work. To be consistent with their work, we would expect the mass in substructures to be higher at higher redshift, if we could add more clusters to our sample and divide them into several bins within the $z=0.4-0.9$ range.

In comparison, Baldi et al. (2012) find no change in the temperature profile, over the redshift range that they broke their sample into (above and below 0.4), implying that there is little change in shape over this redshift range, in apparent contradiction (in terms of a trend) to what we found. However, Baldi et al. (2012) did not have enough data points to subdivide their $z=0.4-0.9$ cluster results into smaller redshift bins for a direct comparison to our observations. Also, as by-product of fitting the X-ray data with a simple beta model, we found an increase in the physical extent (i.e. a larger core radius) of the X-ray surface brightness with decreasing redshift. We judge that this effect is not due to an inability to detect more extended emission at higher redshift, based on the analysis presented in Sect. 4.1.

Our findings are consistent in a general way with the hierarchical cluster growth scenario in that the extent of clusters apparently grows with decreasing redshift. However, if cluster temperature is a valid measure of the cluster mass (independent of redshift), then the fact that we found no relationship between cluster extent and temperature would argue for the mass not having grown significantly over the redshift range from 0.9 to 0.4 , as also indicated by our finding no correlation between the total mass estimated from the X-ray temperature (given in the last column of Table 1) and the redshift. This is plausible if (a) the number of groups infalling over this time period of about 3.6 Gyr is relatively small, and (b) at the same time, the infalling subgroups have caused the ICM to become more extended but not hotter or significantly more massive.

\section{Summary and conclusions}

By the means of a comparison with Takey et al. (2011), we showed that our X-ray luminosities and temperatures were consistant with literature studies. We estimated the substructure detection efficiency with simulations for the X-ray and SG methods. The X-ray detections proved to be efficient up to $z \sim 0.9$ for substructures brighter than $1.0 \times 10^{44} \mathrm{erg} / \mathrm{s}$ and up to $z \sim 0.5$ for substructures only brighter than $0.4 \times 10^{44} \mathrm{erg} / \mathrm{s}$.

The SG detection efficiency was tested by considering six reference clusters outside of our sample, all very well sampled spectroscopically. Substructures with more than six members in the original spectroscopic catalogues remained detected by the SG analysis down to completenesses of $50 \%$ to $30 \%$. We showed that the SG precision on the mass estimate remained better than $50 \%$ for about half of the sample down to incompletenesses of about $60 \%$, while the SG analysis was not able to precisely estimate the mass of most of the substructures for completeness levels lower than $50 \%$. SG masses were also compared to an optically based cluster mass determination, and we found qualitative agreement. We emphasize, however, that only relative SG masses should be considered as reliable.

We found that the core radius of the X-ray gas density profile may decrease with redshift, but this needs to be confirmed with a larger sample of clusters. Ten substructures were detected by both methods (X-rays and SG). These were systematically the SG most massive substructures in each cluster. For a given cluster, the percentage of mass included in substructures was roughly constant with redshift at values of $\sim 5-15 \%$. We also showed that most of our substructures detected both in X-rays and with the SG method were probably at their first cluster pericentre approach and therefore corresponded to relatively recent infalls. 
Finally, compared to regular cluster galaxies, galaxy populations in substructures exhibit a possible lack of both late type and old stellar population galaxies, and a possible lack of late type galaxies with recent bursts of star formation in the substructures closest to the mean cluster velocity. In general, our results are consistent with the picture of CDM hierarchical structure formation in that substructure exists. The approximate $\mathrm{X}$-ray properties and masses of the substructures relative to the entire clusters are in the range predicted by theory: $5-15 \%$, see Gao et al. (2012) for the fraction found at $z=0$, and by the Millennium simulation, which predicts that clusters below $z \sim 1$ only undergo minor mergers. On the simulation front, the percentage of the substructure mass relative to the total mass of the cluster would be interesting to compare with the data we have presented here, as well as with future increased samples produced by our DAFT/FADA collaboration and others.

Acknowledgements. We thank the referee for useful comments. We gratefully acknowledge financial support from the Centre National d'Etudes Spatiales for many years. This project has benefitted from CAPES/COFECUB (programme 711/11). IM has been partially funded by projects AYA2010-15169 from the Spanish Ministerio de Ciencia e Innovación and TIC 114 and PO08-TIC3531 from Junta de Andalucía. We thank Calar Alto Observatory for allocation of director's discretionary time to this programme. Based on XMM-Newton archive data and on data retrieved from the NASA/IPAC Extragalactic Database (NED), which is operated by the Jet Propulsion Laboratory, California Institute of Technology, under contract with the National Aeronautics and Space Administration. The scientific results reported in this article are also based in part on data obtained from the Chandra Data Archive. Based on observations made with the FORS2 multi-object spectrograph mounted on the Antu VLT telescope at ESO-Paranal Observatory (programmes 085.A-0016, 191.A-0268; PI: C. Adami). Also based on observations obtained at the Gemini Observatory, which is operated by the Association of Universities for Research in Astronomy, Inc., under a cooperative agreement with the NSF on behalf of the Gemini partnership: the National Science Foundation (United States), the Science and Technology Facilities Council (United Kingdom), the National Research Council (Canada), CONICYT (Chile), the Australian Research Council (Australia), Ministério da Ciência, Tecnologia e Inovação (Brazil), and Ministerio de Ciencia, Tecnología e Innovación Productiva (Argentina). Also based on observations made with the Italian Telescopio Nazionale Galileo (TNG) operated on the island of La Palma by the Fundación Galileo Galilei of the INAF (Istituto Nazionale di Astrofisica) at the Spanish Observatorio del Roque de los Muchachos of the Instituto de Astrofísica de Canarias. Also based on service observations made with the WHT operated on the island of La Palma by the Isaac Newton Group in the Spanish Observatorio del Roque de los Muchachos of the Instituto de Astrofísica de Canarias. Also based on observations collected at the GermanSpanish Astronomical Center, Calar Alto, jointly operated by the Max-PlanckInstitut fur Astronomie Heidelberg and the Instituto de Astrofísica de Andalucía (CSIC). Based on observations obtained with MegaPrime/MegaCam, a joint project of CFHT and CEA/IRFU, at the Canada-France-Hawaii Telescope (CFHT) which is operated by the National Research Council (NRC) of Canada, the Institut National des Sciences de l'Univers of the Centre National de la Recherche Scientifique (CNRS) of France, and the University of Hawaii. This work is based in part on data products produced at Terapix available at the Canadian Astronomy Data Centre as part of the Canada-France-Hawaii Telescope Legacy Survey, a collaborative project of NRC and CNRS. Also based on observations obtained at the WIYN telescope (KNPO). The WIYN Observatory is a joint facility of the University of Wisconsin-Madison, Indiana University, Yale University, and the National Optical Astronomy Observatory. Kitt Peak National Observatory, National Optical Astronomy Observatory, is operated by the Association of Universities for Research in Astronomy (AURA) under cooperative agreement with the National Science Foundation. Also based on observations obtained at the MDM observatory ( $2.4 \mathrm{~m}$ telescope). MDM consortium partners are Columbia University Department of Astronomy and Astrophysics, Dartmouth College Department of Physics and Astronomy, University of Michigan Astronomy Department, The Ohio State University Astronomy Department, Ohio University Dept. of Physics and Astronomy. Also based on observations obtained at the Southern Astrophysical Research (SOAR) Telescope, which is a joint project of the Ministerio da Ciência, Tecnologia, e Inovação (MCTI) da República Federativa do Brasil, the US National Optical Astronomy Observatory (NOAO), the University of North Carolina at Chapel Hill (UNC), and Michigan State University (MSU). Also based on observations obtained at the Cerro Tololo Inter-American Observatory, National Optical Astronomy Observatory, which are operated by the Association of Universities for Research in Astronomy, under contract with the National Science Foundation. Finally, this research has made use of the VizieR catalogue access tool, CDS, Strasbourg, France.

\section{References}

Abell, G. O., Corwin, H. G. Jr, \& Olowin, R. P. 1989, ApJS, 70, 1 Adami, C., Mazure, A., Biviano, A., Katgert, P., \& Rhee, G. 1998, A\&A, 331, 493

Adami, C., Biviano, A., Durret, F., \& Mazure, A. 2005, A\&A, 443, 17

Adami, C., Pellò, R., Ulmer, M. P., et al. 2009a, A\&A, 495, 407

Adami, C., Le Brun, V., Biviano, A., et al. 2009b, A\&A, 507, 1225

Adami, C., Durret, F., Benoist, C., et al. 2010, A\&A, 509, A81

Adami, C., Jouvel, S., Guennou, L., et al. 2012, A\&A, 540, A105

Adami, C., Durret, F., Guennou, L., \& Da Rocha, C. 2013, A\&A, 551, A20

Allen, S. W., Evrard, A. E., \& Mantz, A. B. 2011, ARA\&A, 49, 409

Andrade-Santos, F., Lima Neto, G. B., \& Laganá, T. F. 2012, ApJ, 746, 139

Baldi, A., Ettori, S., Molendi, S., \& Gastaldello, F. 2012, A\&A, 545, A41

Barkhouse, W. A., Green, P. J., Vikhlinin, A., et al. 2006, ApJ, 645, 955

Bell, E. F., McIntosh, D. H., Katz, N., \& Weinberg, M. D. 2003, ApJS, 149, 289

Böhringer, H., Briel, U. G., Schwarz, R. A., et al. 1994, Nature, 368, 828

Böhringer, H., Pratt, G. W., Arnaud, M., et al. 2010, A\&A, 514, A32

Borgani, S., \& Kravtsov, A. 2011, Adv. Sci. Lett., 4, 204

Boué, G. Durret, F., Adami, C., et al. 2008, A\&A, 489, 11

Bruzual, G., \& Charlot, S. 2003, MNRAS, 344, 1000

Cappellari, M., Bacon, R., Bureau, M., et al. 2006, MNRAS, 366, 1126

Carlberg, R. G., Yee, H. K. C., Morris, S. L., et al. 2001, ApJ, 552, 427

Chon, G., Böhringer, H., \& Smith, G. P. 2012, A\&A, 548, A59

Clowe, D. I., Luppino, G. A., Kaiser, N., Henry, J. P., \& Gioia, I. M. 1998, ApJ, 497, L61

Clowe, D., Schneider, P., Aragón-Salamanca, A., et al. 2006, A\&A, 451, 395

Connelly, J. L., Wilman, D. J., Finoguenov, A., et al. 2012, ApJ, 756, 139

Churazov, E., Forman, W., Jones, C., \& Böhringer, H. 2003, ApJ, 590, 225

Dawson, S., Stern, D., Bunker, A. J., Spinrad, H., \& Dey, A. 2001, AJ, 122, 598

De Filippis, E., Schindler, S., \& Castillo-Morales, A. 2003, A\&A, 404, 63

Dressler, A., \& Schechtman, S. A. 1988, AJ, 95, 985

Dunkley, J., Komatsu, E., Nolta, M. R., et al. 2009, ApJS, 180, 306

Dupke, R. A., \& Bregman, J. N. 2001, ApJ, 547, 705

Durret, F., Adami, C., Gerbal, D., \& Pislar, V. 2000, A\&A, 356, 815

Durret, F., Slezak, E., \& Adami, C. 2009, A\&A, 506, 637

Durret, F., Laganá, T. F., Adami, C., \& Bertin, E. 2010, A\&A, 517, A94

Ebeling, H., Edge, A. C., \& Henry, J. P. 2001a, ApJ, 553, 668

Ebeling, H., Jones, L. R., Fairley, B. W., et al. 2001b, ApJ, 548, L23

Ebeling, H., Barrett, E., Donovan, D., et al. 2007, ApJ, 661, L33

Ebeling, H., Edge, A. C., Mantz, A., et al. 2010, MNRAS, 407, 83

Eckmiller, H. J., Hudson, D. S., \& Reiprich, T. H. 2011, A\&A, 535, A105

Einasto, M., Vennik, J., Nurmi, P., et al. 2012, A\&A, 540, A123

Fukugita, M., Shimasaku, K., \& Ichikawa, T. 1995, PASP, 107, 945

Furusho, T., Yamasaki, N. Y., Ohashi, T., Shibata, R., \& Ezawa, H. 2001, ApJ, $561, \mathrm{~L} 165$

Gal, R. R., \& Lubin, L. M. 2004, ApJ, 607, L1

Gal, R. R., Lubin, L. M., \& Squires, G. K. 2005, AJ, 129, 1827

Gal, R. R., Lemaux, B. C., Lubin, L. M., Kocevski, D., \& Squires, G. K. 2008, ApJ, 684, 933

Gao, L., Navarro, J. F., Frenk, C. S., et al. 2012, MNRAS, 425, 2169

Gerke, B. F., Newman, J. A., Faber, S. M., et al. 2007, MNRAS, 376, 1425

Gilmour, R., Best, P., \& Almaini, O. 2009, MNRAS, 392, 1509

Giodini, S., Pierini, D., Finoguenov, A., et al. 2009, ApJ, 703, 982

Gioia, I. M., Maccacaro, T., Schild, R. E., et al. 1990, ApJS, 72, 567

Gioia, I. M., Henry, J. P., Mullis, C. R., Ebeling, H., \& Wolter, A. 1999, AJ, 117, 2608

Gioia, I. M., Braito, V., Branchesi, M., et al. 2004, A\&A, 419, 517

Girardi, M., Demarco, R., Rosati, P., \& Borgani, S. 2005, A\&A, 442, 29

Gladders, M. D., \& Yee, H. K. C. 2005, ApJS, 157, 1

Gonzalez, A. H., Zaritsky, D., Dalcanton, J. J., \& Nelson, A. 2001, ApJS, 137, 117

Gonzalez, A. H., Zaritsky, D., \& Zabludoff, A. I. 2007, ApJ, 666, 147

Green, P. J., Infante, L., Lopez, S., Aldcroft, T. L., \& Winn, J. N. 2005, ApJ, 630, 142

Guennou, L. 2012, Ph.D. Thesis, Université de Marseille-Provence, France

Guennou, L., Adami, C., Ulmer, M. P., et al. 2010, A\&A, 523, A21

Guennou, L., Biviano, A., Adami, C., et al. 2013, A\&A, submitted

Gunn, J. E., Hoessel, J. G., \& Oke, J. B. 1986, ApJ, 306, 30

Halliday, C., Milvang-Jensen, B., Poirier, S., et al. 2004, A\&A, 427, 397

Harrison, F. A., Eckart, M. E., Mao, P. H., Helfand, D. J., \& Stern, D. 2003, ApJ, 596,944 
Henry, J. P., Gioia, I. M., \& Mullis, C. R. 1997, AJ, 114, 1293 Holden, B. P., Stanford, S. A., Rosati, P., et al. 2001, AJ, 122, 629 Jee, M. J., \& Tyson, J. A. 2009, ApJ, 691, 1337

Jones, C., \& Forman, W. 1984, ApJ, 276, 38

Kauffmann, G., Heckman, T. M., White, S. D. M., et al. 2003, MNRAS, 341, 33 Kolokotronis, V. Georgakakis, A., Basilakos, S., et al. 2006, MNRAS, 366, 163 Komatsu, E., Matsuo, H., Kitayama, T., et al. 2001, PASJ, 53, 57 Korngut, P. M., Dicker, S. R., Reese, E. D., et al. 2011, ApJ, 734, 10 Kravtsov, A. V., \& Borgani, S. 2012, ARA\&A, 50, 353

Kravtsov, A., Vikhlinin, A., \& Nagai, D. 2006, ApJ, 650, 128

Lilly, S. J., LeBrun, V., Maier, C., et al. 2009, ApJS, 184, 218

Lopes, P. A. A., de Carvalho, R. R., Capelato, H. V., et al. 2006, ApJ, 648, 209

Mamon, G. A. 2000, ASPC, 197, 377

Mann, A. W., \& Ebeling, H. 2012, MNRAS, 420, 2120

Mathur, S., \& Williams, R. J. 2003, ApJ, 589, L1

Maughan, B. J., Jones, C., Jones, L.R.., \& Van Speybroeck, L. 2007, ApJ, 659, 1125

Maughan, B. J., Jones, C., Forman, W., \& Van Speybroeck, L. 2008, ApJS, 174, 117

McHardy, I. M., Jones, L. R., Merrifield, M. R., et al. 1998, MNRAS, 295, 641

Milvang-Jensen, B., Noll, S., Halliday, C., et al. 2008, A\&A, 482, 419

Moran, S. M., Ellis, R. S., Treu, T., et al. 2007, ApJ, 671, 1503

Mulchaey, J. S., Lubin, L. M., Fassnacht, C., Rosati, P., \& Jeltema, T. E. 2006, ApJ, 646, 133

Navarro, J. F., Ludlow, A., Springel, V., et al. 2010, MNRAS, 402, 21

Neumann, D. M., Lumb, D. H., Pratt, G. W., \& Briel, U. G. 2003, A\&A, 400, 811

Perlman, E. S., Horner, D. J., Jones, L. R., et al. 2002, ApJS, 140, 265

Poole, G. B., Babul, A., McCarthy, I. G., et al. 2007, MNRAS, 380, 437

Postman, M., Coe, D., Benítez, N., et al. 2012, ApJS, 199, 25

Schindler, S., Guzzo, L., Ebeling, H., et al. 1995, A\&A, 299, L9

Schirmer, M., Suyu, S., Schrabback, T., et al. 2010, A\&A, 514, A60

Serna, A., \& Gerbal, D. 1996, A\&A, 309, 65

Shibata, R., Matsushita, K., Yamasaki, N. Y., et al. 2001, ApJ, 549, 228

Smith, G. P., Khosroshahi, H. G., Dariush, A., et al. 2010, MNRAS, 409, 169

Springel, V., White, S. D. M., Jenkins, A., et al. 2005, Nature, 435, 629

Springel, V., White, S. D. M., Frenk, C. S., et al. 2008a, Nature, 456, 73

Springel, V., Wang, J., Vogelsberger, M., et al. 2008b, MNRAS, 391, 1685

Strigari, L. E., Frenk, C. S., \& White, S. D. M. 2010, MNRAS, 408, 2364

Takey, A., Schwope, A., \& Lamer, G. 2011, A\&A, 534, A120

Takizawa, M. 2005, ApJ, 629, 791

Tonnesen, S., \& Bryan, G. L. 2008, ApJ, 684, L9

Tremonti, C. A., Heckman, T. M., Kauffmann, G., et al. 2004, ApJ, 613, 898

Ulmer, M. P., Adami, C., Covone, G., et al. 2005, ApJ, 624, 124

Ulmer, M. P., Adami, C., Lima Neto, G. B., et al. 2009, A\&A, 503, 399

Vikhlinin, A., McNamara, B. R., Forman, W., et al. 1998, ApJ, 502, 558

Vikhlinin, A., Kravtsov, A., Forman, W., et al. 2006, ApJ, 640, 691

Vikhlinin, A., Murray, S., Gilli, R., et al. 2009, Astro2010: The Astronomy and Astrophysics Decadal Survey (Science White Papers), 305

[arXiv:0903.5320]
Voges, W., Aschenbach, B., Boller, T., et al. 1999, A\&A, 349, 389 Weissmann, A., Böhringer, H., \& Chon, G. 2013, A\&A, 555, A147 Wen, Z. L., \& Han, J. L. 2013, MNRAS, 436, 275

Xue, Y. J., \& Wu, X. P. 2000, ApJ, 538, 65

1 Aix-Marseille Université, CNRS, LAM (Laboratoire d'Astrophysique de Marseille) UMR 7326, 13388 Marseille, France e-mail: guennou@ukzn.ac.za

2 Astrophysics and Cosmology Research Unit, University of KwaZulu-Natal, 4041 Durban, South Africa

3 UPMC-CNRS, UMR7095, Institut d'Astrophysique de Paris, 75014 Paris, France

${ }^{4}$ Departamento de Astronomia, Instituto de Astronomia, Geofísica e Ciências Atmosféricas, Universidade de São Paulo, Rua do Matão 1226, 05508-900 São Paulo, Brazil

5 Dept of Physics and Astronomy \& Center for Interdisciplinary Exploration and Research in Astrophysics (CIERA), Evanston IL 60208-2900, USA

${ }^{6}$ Department of Physics and Astronomy, Ohio University, 251B Clippinger Lab, Athens OH 45701, USA

7 Fermi National Accelerator Laboratory, PO Box 500, Batavia IL 60510, USA

${ }^{8}$ CSC/STScI, 3700 San Martin Dr., Baltimore MD 21218, USA

9 OCA, Cassiopée, Boulevard de l'Observatoire, BP 4229, 06304 Nice Cedex 4, France

10 INAF/Osservatorio Astronomico di Trieste, via Tiepolo 11, 34143 Trieste, Italy

11 INAF - Osservatorio Astronomico di Bologna, via Ranzani 1, 40127 Bologna, Italy

1223 rue d'Yerres, 91230 Montgeron, France

13 Steward Observatory, University of Arizona, 933 N. Cherry Ave., Tucson AZ 85721, USA

14 Department of Astronomy \& Astrophysics, University of Toronto, 50 St George Street, Toronto M5S 3H4, Canada

15 Instituto de Astrofísica de Andalucía, CSIC, Glorieta de la Astronomía s/n, 18008 Granada, Spain

${ }^{16}$ Laboratório de Astrofísica Teórica e Observacional, Universidade Estadual de Santa Cruz, 45662-000 Ilhéus, Brazil

17 Gemini Observatory, 603 Casilla, La Serena, Chile

18 Argelander-Institut für Astronomie, Universität Bonn, auf dem Hügel 71, 53121 Bonn, Germany

19 University of Vienna, Department of Astronomy, Türkenschanzstrasse 17, 1180 Vienna, Austria 


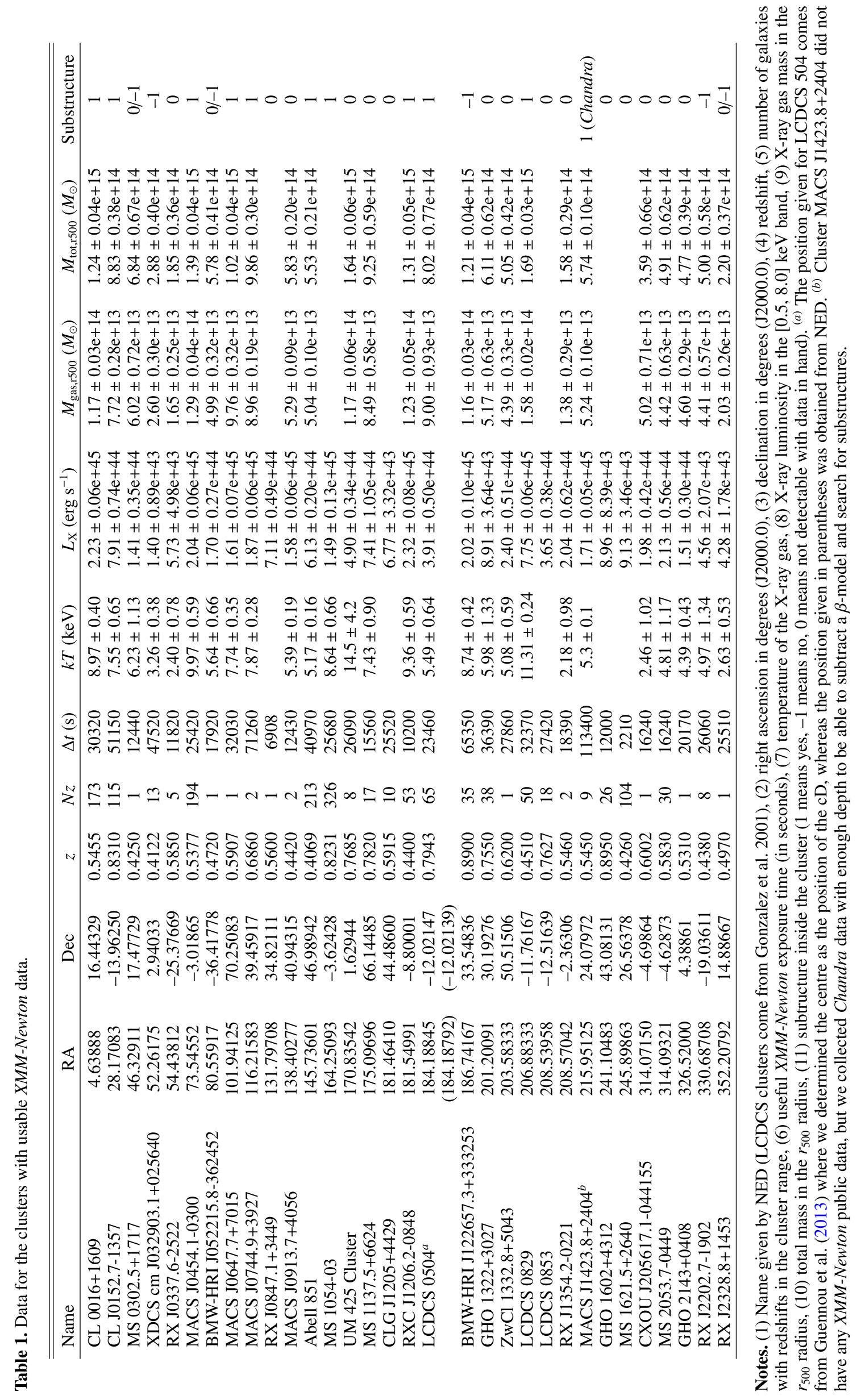


Table 2. Detected substructures.

\begin{tabular}{|c|c|c|c|c|c|c|c|c|}
\hline Name & $\#$ & $N_{\text {gal }}$ & $\mathrm{z}$ & $\begin{array}{r}\left(M_{\mathrm{SS}} / M_{\mathrm{tot}}\right)_{\mathrm{SG}} \\
(\%)\end{array}$ & $\begin{array}{r}\left(M_{\mathrm{SS}} / M_{\mathrm{tot}}\right)_{\mathrm{sc}} \\
(\%)\end{array}$ & $\begin{array}{r}\text { Vel. disp. } \\
\left(\mathrm{km} \mathrm{s}^{-1}\right)\end{array}$ & $\begin{array}{r}L_{X} \\
(\mathrm{erg} / \mathrm{s}) \\
\end{array}$ & $\begin{array}{r}\text { Merging } \\
\text { stage }\end{array}$ \\
\hline \multirow{4}{*}{ CL 0016+1609 } & 1 & 64 & 0.5418 & {$[10 ; 20]$} & {$[10 ; 20]$} & & & \\
\hline & 2 & 17 & 0.5505 & {$[0 ; 10]$} & {$[0 ; 10]$} & & & \\
\hline & 3 & 13 & 0.5530 & {$[0 ; 10]$} & {$[0 ; 10]$} & & & \\
\hline & 4 & 24 & 0.5597 & {$[0 ; 10]$} & {$[0 ; 10]$} & 200 & $1.56 \times 10^{44}$ & $\mathrm{t} 1$ \\
\hline \multirow[t]{4}{*}{ CL J0152.7-1357 } & 1 & 49 & 0.8382 & {$[20 ; 30]$} & {$[40 ; 50]$} & 680 & $3.28 \times 10^{44}$ & $\mathrm{t} 1$ \\
\hline & 2 & 17 & 0.8323 & {$[0 ; 10]$} & {$[0 ; 10]$} & & & \\
\hline & 3 & & 0.8279 & {$[0 ; 10]$} & {$[0 ; 10]$} & & & \\
\hline & 4 & 34 & 0.8458 & {$[20 ; 30]$} & {$[0 ; 10]$} & 320 & $2.27 \times 10^{44}$ & $\mathrm{t} 1$ \\
\hline XDCS cm J032903.1+025640 & 1 & 7 & 0.4115 & {$[0 ; 10]$} & {$[50 ; 60]$} & & & \\
\hline & 2 & 4 & 0.4095 & {$[0 ; 10]$} & {$[0 ; 10]$} & & & \\
\hline \multirow[t]{8}{*}{ MACS J0454.1-0300 } & 1 & 31 & 0.5365 & {$[10 ; 20]$} & {$[0 ; 10]$} & & & \\
\hline & 2 & $\begin{array}{r}5 \\
18\end{array}$ & $\begin{array}{l}0.5407 \\
0.5434\end{array}$ & $\begin{array}{l}{[0 ; 10]} \\
{[0 \cdot 10]}\end{array}$ & $\begin{array}{l}{[0 ; 10]} \\
{[0 \cdot 10]}\end{array}$ & 320 & $3.24 \times 10^{44}$ & t1 \\
\hline & 4 & $\begin{array}{r}10 \\
6\end{array}$ & 0.5376 & {$[0 ; 10]$} & {$[0: 10]$} & & & [I \\
\hline & 5 & 3 & 0.5320 & {$[0 ; 10]$} & {$[0 ; 10]$} & & & \\
\hline & 6 & 12 & 0.5309 & {$[0 ; 10]$} & {$[0 ; 10]$} & & & \\
\hline & 7 & 3 & 0.5390 & {$[0 ; 10]$} & {$[0 ; 10]$} & & & \\
\hline & 8 & 4 & 0.5457 & {$[0 ; 10]$} & {$[0 ; 10]$} & & & \\
\hline & 9 & 4 & 0.5287 & {$[0 ; 10]$} & {$[0 ; 10]$} & & & \\
\hline \multirow{8}{*}{ Abell 851} & 1 & 6 & 0.4070 & {$[0 ; 10]$} & {$[0 ; 10]$} & & & \\
\hline & 2 & 3 & 0.4100 & {$[0 ; 10]$} & {$[0 ; 10]$} & & & \\
\hline & 3 & 4 & 0.4036 & {$[0 ; 10]$} & {$[0 ; 10]$} & 1300 & $5.63 \times 10^{43}$ & $\mathrm{t} 0(\mathrm{t} 1$ or $\mathrm{t} 2)$ \\
\hline & 4 & 3 & 0.4059 & {$[0 ; 10]$} & {$[0 ; 10]$} & & & \\
\hline & 5 & 4 & 0.4100 & {$[0 ; 10]$} & {$[0 ; 10]$} & & & \\
\hline & 6 & 8 & 0.4142 & {$[0 ; 10]$} & {$[0 ; 10]$} & & & \\
\hline & 7 & 3 & 0.4100 & {$[0 ; 10]$} & {$[0 ; 10]$} & & & \\
\hline & 8 & 3 & 0.4163 & {$[0 ; 10]$} & {$[0 ; 10]$} & & & \\
\hline \multirow[t]{4}{*}{ MS 1054-03 } & 1 & 7 & 0.8218 & {$[0 ; 10]$} & {$[0 ; 10]$} & 1250 & $1.94 \times 10^{44}$ & $\mathrm{t} 1(\mathrm{t} 0$ or $\mathrm{t} 2)$ \\
\hline & 2 & 26 & 0.8261 & {$[0 ; 10]$} & {$[0 ; 10]$} & & & \\
\hline & 3 & 5 & 0.8267 & {$[0 ; 10]$} & {$[0 ; 10]$} & & & \\
\hline & 4 & 5 & 0.8270 & {$[0 ; 10]$} & {$[0 ; 10]$} & & & \\
\hline \multirow{7}{*}{ BMW-HRI J122657.3+333253 } & 1 & 11 & 0.5948 & {$[90 ; 100]^{*}$} & {$[90 ; 100]$} & & & \\
\hline & 1 & 10 & 0.8816 & {$[20 ; 30]$} & {$[0 ; 10]$} & & & \\
\hline & 2 & 4 & 0.8910 & {$[0 ; 10]$} & {$[0 ; 10]$} & & & \\
\hline & 3 & 5 & 0.8920 & {$[0 ; 10]$} & {$[0 ; 10]$} & & & \\
\hline & 4 & 5 & 0.8930 & {$[0 ; 10]$} & {$[0 ; 10]$} & & & \\
\hline & 5 & 4 & 0.8960 & {$[0 ; 10]$} & {$[0 ; 10]$} & & & \\
\hline & 6 & 4 & 0.8970 & {$[0 ; 10]$} & {$[0 ; 10]$} & & & \\
\hline \multirow[t]{4}{*}{ RXC J1206.2-0848 } & 1 & 5 & 0.4255 & {$[0 ; 10]$} & {$[0 ; 10]$} & & & \\
\hline & 2 & 3 & 0.4336 & {$[0 ; 10]$} & {$[0 ; 10]$} & & & \\
\hline & 3 & 6 & 0.4409 & {$[0 ; 10]$} & {$[0 ; 10]$} & & & \\
\hline & 4 & 4 & 0.4373 & {$[0 ; 10]$} & {$[0 ; 10]$} & 690 & $5.10 \times 10^{43}$ & $\mathrm{t} 1(\mathrm{t} 0$ or $\mathrm{t} 2)$ \\
\hline \multirow[t]{7}{*}{ LCDCS 0504} & 1 & 7 & 0.8036 & {$[0 ; 10]$} & {$[0 ; 10]$} & 110 & $3.10 \times 10^{43}$ & $\mathrm{t} 1(\mathrm{t} 0$ or $\mathrm{t} 2)$ \\
\hline & 2 & 10 & 0.7996 & {$[0 ; 10]$} & {$[0 ; 10]$} & & & \\
\hline & 3 & 17 & 0.7858 & {$[0 ; 10]$} & {$[0 ; 10]$} & & & \\
\hline & 4 & 5 & 0.7913 & {$[0 ; 10]$} & {$[0 ; 10]$} & & & \\
\hline & 5 & 6 & 0.7966 & {$[0 ; 10]$} & {$[0 ; 10]$} & & & \\
\hline & 6 & 7 & 0.7953 & {$[0 ; 10]$} & {$[0 ; 10]$} & & & \\
\hline & 7 & 6 & 0.7940 & {$[0 ; 10]$} & {$[0 ; 10]$} & & & \\
\hline GHO $1322+3027$ & 1 & 44 & 0.7562 & {$[90 ; 100]^{*}$} & {$[90 ; 100]$} & & & \\
\hline \multirow{4}{*}{ LCDCS 0829} & 1 & 22 & 0.4503 & {$[0 ; 10]$} & {$[40 ; 50]$} & 230 & $5.79 \times 10^{44}$ & $\mathrm{t} 1$ \\
\hline & 2 & 14 & 0.4529 & {$[0 ; 10]$} & {$[20 ; 30]$} & & & \\
\hline & 3 & 15 & 0.4465 & {$[0 ; 10]$} & {$[0 ; 10]$} & & & \\
\hline & 4 & 12 & 0.4553 & {$[0 ; 10]$} & {$[0 ; 10]$} & 180 & $1.82 \times 10^{45}$ & $\mathrm{t} 1$ \\
\hline LCDCS 0853 & 1 & 3 & 0.7648 & {$[90 ; 100]^{*}$} & {$[20 ; 30]$} & & & \\
\hline MACS J1423.8+2404 & 1 & 3 & 0.5445 & {$[90 ; 100] *$} & {$[30 ; 40]$} & & & \\
\hline GHO $1602+4312$ & 1 & 29 & 0.8941 & {$[90 ; 100] *$} & {$[90 ; 100]$} & & & \\
\hline \multirow[t]{4}{*}{ MS $1621.5+2640$} & 1 & 24 & 0.4245 & {$[0 ; 10]$} & {$[10 ; 20]$} & & & \\
\hline & 2 & 19 & 0.4264 & {$[0 ; 10]$} & {$[0 ; 10]$} & & & \\
\hline & 3 & 43 & 0.4307 & {$[10 ; 20]$} & {$[20 ; 30]$} & & & \\
\hline & 4 & 20 & 0.4211 & {$[0 ; 10]$} & {$[0 ; 10]$} & & & \\
\hline MS 2053.7-0449 & 1 & 28 & 0.5837 & {$[90 ; 100]^{*}$} & {$[90 ; 100]$} & & & \\
\hline GHO $2143+0408$ & 1 & 4 & 0.5205 & {$[90 ; 100] *$} & {$[80 ; 90]$} & & & \\
\hline
\end{tabular}

Notes. (1) Cluster name, (2) substructure number, (3) substructure number of galaxies, (4) substructure mean redshift, (5) substructure to total cluster mass ratio estimated with the SG method and given in $10 \%$ wide intervals (the asterisk means that we only detected the main structure), (6) substructure to total cluster mass ratio estimated with the method based on a scaling relation described in Sect. 3.5 and given in $10 \%$ wide intervals, (7) substructure velocity dispersion estimated with SG for the substructures also detected in X-rays, (8) substructure X-ray luminosity, (9) merging stage (see text). 
L. Guennou et al.: Substructures in $0.4<z<0.9$ clusters

Table 4. Optical structures found in the clusters with no X-ray data.

\begin{tabular}{|c|c|c|c|c|c|}
\hline Name & $\#$ & $N_{\text {gal }}$ & $\mathrm{z}$ & $\begin{array}{c}\left(M_{\mathrm{SS}} / M_{\mathrm{tot}}\right)_{\mathrm{SG}} \\
(\%)\end{array}$ & $\begin{array}{c}\left(M_{\mathrm{SS}} / M_{\mathrm{tot}}\right)_{\mathrm{sc}} \\
(\%)\end{array}$ \\
\hline \multirow[t]{3}{*}{ CXOMP J091126.6+055012 } & 1 & 11 & 0.7687 & {$[20 ; 30]$} & {$[30 ; 40]$} \\
\hline & 2 & 7 & 0.7623 & {$[0 ; 10]$} & {$[10 ; 20]$} \\
\hline & 3 & 6 & 0.7748 & {$[0 ; 10]$} & {$[0 ; 10]$} \\
\hline \multirow[t]{2}{*}{ LCDCS 0110} & 1 & 4 & 0.5807 & {$[0 ; 10]$} & {$[10 ; 20]$} \\
\hline & 2 & 9 & 0.5777 & {$[0 ; 10]$} & {$[40 ; 50]$} \\
\hline \multirow[t]{3}{*}{ LCDCS 0130} & 1 & 7 & 0.7041 & {$[0 ; 10]$} & {$[0 ; 10]$} \\
\hline & 2 & 5 & 0.7028 & {$[0 ; 10]$} & {$[0 ; 10]$} \\
\hline & 3 & 8 & 0.7059 & {$[0 ; 10]$} & {$[0 ; 10]$} \\
\hline \multirow[t]{3}{*}{ LCDCS 0172} & 1 & 24 & 0.6977 & {$[0 ; 10]$} & {$[30 ; 40]$} \\
\hline & 2 & 6 & 0.6979 & {$[0 ; 10]$} & {$[0 ; 10]$} \\
\hline & 3 & 7 & 0.6944 & {$[0 ; 10]$} & {$[0 ; 10]$} \\
\hline \multirow[t]{4}{*}{ LCDCS 0173} & 1 & 12 & 0.7498 & {$[0 ; 10]$} & {$[10 ; 20]$} \\
\hline & 2 & 11 & 0.7477 & {$[0 ; 10]$} & {$[10 ; 20]$} \\
\hline & 3 & 8 & 0.7523 & {$[0 ; 10]$} & {$[0 ; 10]$} \\
\hline & 4 & 5 & 0.7573 & {$[10 ; 20]$} & {$[0 ; 10]$} \\
\hline \multirow[t]{2}{*}{ CXOMP J111726.1+074335 } & 1 & 22 & 0.4833 & {$[0 ; 10]$} & {$[30 ; 40]$} \\
\hline & 2 & 16 & 0.4790 & {$[0 ; 10]$} & {$[10 ; 20]$} \\
\hline \multirow[t]{6}{*}{ LCDCS 340} & 1 & 9 & 0.4852 & {$[0 ; 10]$} & {$[0 ; 10]$} \\
\hline & 2 & 5 & 0.4765 & {$[0 ; 10]$} & {$[0 ; 10]$} \\
\hline & 3 & 5 & 0.4818 & {$[0 ; 10]$} & {$[0 ; 10]$} \\
\hline & 4 & 4 & 0.4801 & {$[0 ; 10]$} & {$[0 ; 10]$} \\
\hline & 5 & 4 & 0.4787 & {$[0 ; 10]$} & {$[0 ; 10]$} \\
\hline & 6 & 5 & 0.4796 & {$[0 ; 10]$} & {$[0 ; 10]$} \\
\hline \multirow[t]{8}{*}{ LCDCS 0541} & 1 & 9 & 0.5420 & {$[0 ; 10]$} & {$[0 ; 10]$} \\
\hline & 2 & 11 & 0.5447 & {$[0 ; 10]$} & {$[0 ; 10]$} \\
\hline & 3 & 10 & 0.5395 & {$[0 ; 10]$} & {$[0 ; 10]$} \\
\hline & 4 & 13 & 0.5347 & {$[20 ; 30]$} & {$[0 ; 10]$} \\
\hline & 5 & 7 & 0.5432 & {$[0 ; 10]$} & {$[0 ; 10]$} \\
\hline & 6 & 9 & 0.5408 & {$[0 ; 10]$} & {$[0 ; 10]$} \\
\hline & 7 & 4 & 0.5379 & {$[0 ; 10]$} & {$[0 ; 10]$} \\
\hline & 8 & 12 & 0.5492 & {$[0 ; 10]$} & {$[0 ; 10]$} \\
\hline \multirow[t]{4}{*}{ ClG J1236+6215 } & 1 & 23 & 0.8521 & {$[0 ; 10]$} & {$[30 ; 40]$} \\
\hline & 2 & 17 & 0.8494 & {$[0 ; 10]$} & {$[0 ; 10]$} \\
\hline & 3 & 13 & 0.8495 & {$[0 ; 10]$} & {$[0 ; 10]$} \\
\hline & 4 & 16 & 0.8462 & {$[10 ; 20]$} & {$[10 ; 20]$} \\
\hline \multirow[t]{2}{*}{ 3C 295 Cluster } & 1 & 10 & 0.4560 & {$[0 ; 10]$} & {$[10 ; 20]$} \\
\hline & 2 & 17 & 0.4618 & {$[0 ; 10]$} & {$[20 ; 30]$} \\
\hline \multirow[t]{2}{*}{ GHO 1601+4253 } & 1 & 37 & 0.5392 & {$[0 ; 10]$} & {$[50 ; 60]$} \\
\hline & 2 & 14 & 0.5439 & {$[0 ; 10]$} & {$[0 ; 10]$} \\
\hline
\end{tabular}

Notes. (1) Cluster name, (2) number of the substructure, (3) number of galaxies with redshift in the substructure, (4) mean redshift of the substructure, (5) substructure to total cluster mass ratio estimated with the SG method and given in $10 \%$ wide intervals, (6) substructure to total cluster mass ratio estimated with the method based on a scaling relation described in Sect. 3.5 and given in $10 \%$ wide intervals. 


\section{Appendix A: Substructure analysis of the invidual clusters with usable XMM-Newton and/or Chandra data}

We now describe clusters individually in order of increasing right ascension, focussing on the X-ray and SG analyses. Rounded coordinates (in degrees) and redshifts are given in parentheses for each cluster (in some cases followed by the ? symbol when the cluster redshift is uncertain). For all similar figures in the appendix, the large blue circle shown in the top left figure corresponds to a $500 \mathrm{kpc}$ radius at the cluster redshift, but the centre of this circle is the one given in the literature, and may differ a little from the one we find from our images. In the following, we refer to the $n$th substructure detected by the SG analysis and listed in Table 2 as SGn.

\section{A.1. CL $0016+1609\left(4.63888^{\circ},+16.4433^{\circ}, z=0.5455\right)$}

The subtraction of a $\beta$-model in X-rays shows two X-ray sources in the cluster area and a compact emission north-west of the cluster (identified as an AGN by Gilmour et al. 2009). Following the results of our simulations, the central X-ray source is probably not a real substructure.

The other extended X-ray source (north-east of the cluster) is probably a real substructure of CL $0016+1609$. This X-ray emission does not correspond to any galaxy structure detected on the line of sight (Fig. A.1). Several cluster galaxies belonging to substructures detected by the SG method are present inside this X-ray emission. However, galaxies of groups SG1, SG2, and SG3 are spread over the entire cluster area, while two thirds of the galaxies of SG4 are very close to the X-ray source. We therefore choose to relate SG4 to this extended X-ray source, putting it $\sim 5400 \mathrm{~km} \mathrm{~s}^{-1}$ beyond the cluster main core (group SG1 in Table 2).

The existence of such a substructure is reinforced by the fact that the velocity histogram around $z \sim 0.54$ is clearly asymmetric and seems to show at least three peaks (Fig. A.1).

\section{A.2. CL J0152.7-1357 $\left(28.17083^{\circ},-13.9625^{\circ}, z=0.8310\right)$}

We can see in Fig. A.2 that there are many X-ray sources in the XMM-Newton residual image of this cluster. Part of these sources are due to the interchip gaps (see Fig. A.2). Both the Chandra image and Gilmour et al. (2009) allow us to identify several point sources. Two main extended and highly significant $\mathrm{X}$-ray sources remain, which were previously identified as two major structures in the process of merging (Ebeling et al. 2001b), based on ROSAT data. We are therefore dealing with a major ongoing merger.

The 115 available redshifts in the cluster interval (see Fig. A.2) allow us to characterize this merger. Out of these 115 redshifts, 95 are distributed in four substructures (see Table 2) detected by our SG analysis. Very clearly, substructure SG1 is identified with the extended X-ray residual at the north east and substructure SG4 is identified with the extended X-ray residual at the south west. Substructure SG2 is located between the two extended X-ray emissions and SG3 is probably infalling onto the cluster from the foreground.

We cannot exclude a contamination by a foreground galaxy structure on the line of sight (Fig. A.2), but given the very good correspondence between substructure SG4 and the southwest X-ray emission, we somewhat arbitrarily consider this contamination as negligible. This is consistent with the detailed analysis of Girardi et al. (2005).

\section{A.3. $M S 0302.5+1717\left(46.32911^{\circ},+17.47729^{\circ}, z=0.4250\right)$}

This cluster is rather weak (Gioia et al. 1990 and Fig. A.3). In $\mathrm{X}$-rays, no substructure is detected at better than the $2.5 \sigma$ level when subtracting a $\beta$-model. A single redshift is available in NED so the SG analysis is not possible. X-ray data are, however, deep enough (the Fe line in the X-ray spectrum gives the same redshift for the cluster as the central galaxy: $z=0.426$ ) to be sure that this cluster is not hosting major substructures.

\section{A.4. XDCS cm J032903.1+025640 (52.26175,$+2.94033^{\circ}$, $z=0.4122)$}

The XDCS cm J032903.1+025640 cluster is detected in X-rays (Fig. A.4), but it is rather weak and diffuse, as in the ROSAT image of Mulchaey et al. (2006). After model subtraction, there is hardly any emission left except two residuals probably due to interchip gaps.

There are 13 galaxies in the $[0.40,0.42]$ redshift range. The SG analysis detects two low-mass structures (see Table 2), SG1 probably being identified with the cluster itself. If we consider SG1 and SG2 together, the mass computed by the SG analysis for XDCS cm J032903.1+025640 is $7.10 \times 10^{13} M_{\odot}$.

\section{A.5. $R X J 0337.6-2522\left(54.43812^{\circ},-25.38^{\circ}, z=0.5850\right)$}

We only have limited information for this cluster, which was discovered by Vikhlinin et al. (1998). Its XMM-Newton emission is relatively weak, and neither Chandra data nor catalogues of known active objects in the field are available. There are most probably three X-ray point sources in the field of view (southwest, west, and north-west of the cluster centre, see Fig. A.5). We also detect two small $2.5 \sigma$ level sources in the residual image close to the cluster location, but even the largest one is too faint to provide a successful luminosity measurement. We are therefore probably dealing with a residual of the cluster X-ray emission itself because a $\beta$-model does not perfectly fit the cluster emission.

We only have five galaxy redshifts in the cluster range.

\section{A.6. MACS J0454.1-0300 $\left(73.54552^{\circ},-3.0187^{\circ}, z=0.5377\right)$}

The X-ray image of the well known X-ray cluster MACS J0454.1-0300 (Gioia et al. 1990) appears rather smooth, but after model subtraction a significant excess emission $(4.5 \sigma$ level $)$ is detected about 0.6 arcmin west of the cluster centre (Fig. A.6). It is too extended to be simply due to incorrect modelling by the $\beta$-model. We also detect on a larger scale (not shown on Fig. A.6) $\mathrm{X}$-ray emission that could originate from the fossil group J04540309 at $z=0.26$ analysed by Schirmer et al. (2010). They found that this group was located about 8 arcmin away from MACS J0454.1-0300.

Many redshifts are available in this region, thanks to the spectroscopic observations of Moran et al. (2007), who analysed the transformation of spirals into S0s. The velocity histogram around $z \sim 0.54$ is asymmetric (see Fig. A.6), with several peaks (at least 3); there are 194 galaxies in the [0.52, 0.555] redshift range). Nine substructures are found by the SG analysis (see Table 2). SG1 is probably the one associated with the whole cluster, while SG3 is probably associated with the X-ray residual 

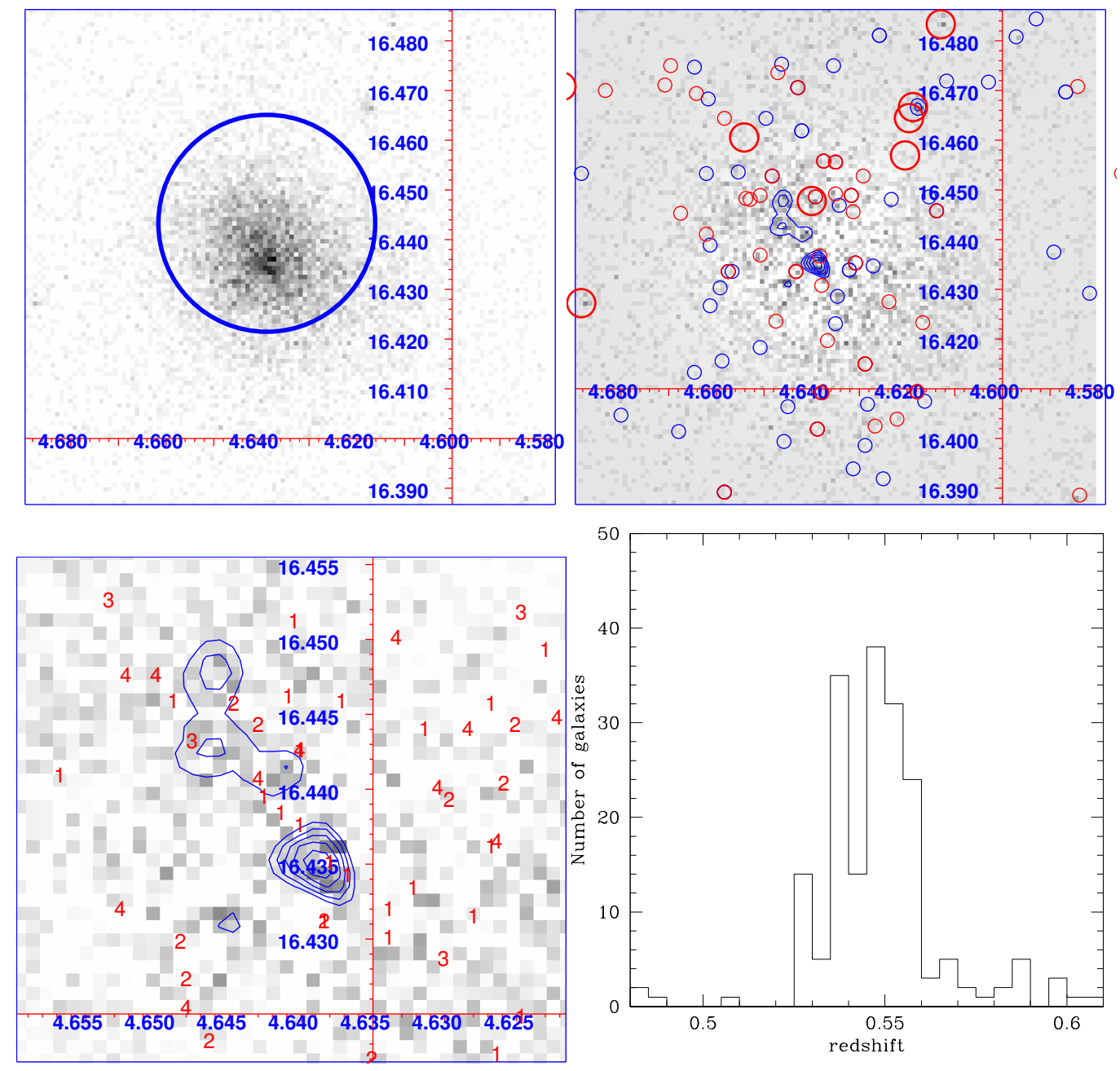

Fig. A.1. XMM-Newton X-ray image (upper left), residual image (upper right), and zoom on the residual image (lower left). The residual image was obtained by subtracting the model from the image for CL $0016+1609$. The large blue circle in the upper left panel corresponds to a $500 \mathrm{kpc}$ radius circle centred on the cluster position found in the literature. The large red circles in the upper right panel show the positions of the known active objects along the line of sight, the small red circles show the positions of the galaxies belonging to structures beyond the cluster from the SG analysis, and the small blue circles show the positions of the galaxies belonging to structures in front of the cluster from the SG analysis. The red numbers in the lower left panel show the positions of the galaxies belonging to substructures in the cluster from the SG analysis, the number being the one given in Table 2. Finally, blue contours in the lower left are the X-ray residuals, starting at the $2.5 \sigma$ level and spaced by $1 \sigma$ intervals. Lower right: redshift histogram for the CL $0016+1609$ area. Note that circles in the upper right panel of this figure (and of the following ones) are sometimes outwith the limits of the image, showing the immediate environment of the cluster considered.

(900 $\mathrm{km} \mathrm{s}^{-1}$ in front of the cluster itself). We are therefore probably observing a collection of several low-mass groups (the most massive being SG3) in the process of merging onto the core of the structure (MACS J0454.1-0300 itself).

\section{A.7. BMW-HRI J052215.8-362452 (80.55917\%, -36.4178 , $Z=0.4720)$}

The residual X-ray image of BMW-HRI J052215.8-362452 (serendipitously discovered by ROSAT, Vikhlinin et al. 1998) shows the presence of several sources superimposed on the cluster, all but one being very compact. The four brightest of these sources are known as AGNs (Gilmour et al. 2009) and are visible in the Chandra image. A fifth one, north-east of the cluster remains unknown and not clearly detected in the Chandra image. The MOS1 interchip separation is also visible (Fig. A.7). Finally, we detect a faint X-ray source to the south (at the $3.5 \sigma$ level), which seems extended in the residual image (Fig. A.7). This could be a substructure both of the BMW-HRI J052215.8362452 cluster itself, even if we cannot be sure of this, because a single redshift is available in NED, and of the vicinity of the interchip gap.

We note that given the strong cluster contamination by AGN and by the MOS1 interchip separation, the X-ray luminosities of the detected substructure are quite uncertain.

\section{A.8. MACS J0647.7+7015 $\left(101.94125^{\circ},+70.2508^{\circ}\right.$, $z=0.5907)$}

The X-ray image of MACS J0647.7+7015 (see e.g. Voges et al. 1999 ) is smooth but some emission is detected after model subtraction to the south-east (at the $3 \sigma$ level: see Fig. A.8). The extension of this possible substructure is 26 arcsec (equivalent to $172 \mathrm{kpc}$ at $z=0.5907)$. There is a single redshift available in the cluster area in NED, so the SG analysis is impossible. Even if we cannot be sure that this substructure is attached to the MACS J0647.7+7015 cluster, its elongation toward the cluster centre tends to indicate that we have detected a substructure in this cluster. Two compact sources are visible in the XMM-Newton data south-west of the cluster. One of them is 



Fig. A.2. XMM-Newton X-ray image (upper left), residual image with straight blue lines showing the interchip detector gaps (upper right), Chandra image (middle left), and zoom on the residual image (middle right). The residual image was obtained by subtracting the model from the XMM-Newton image for CL J0152.7-1357. The large blue circle in the upper left panel corresponds to a $500 \mathrm{kpc}$ radius circle centred on the cluster position found in the literature. The large red circles in the upper right panel show the positions of the known active objects along the line of sight, the small red circles show the positions of the galaxies belonging to structures beyond the cluster from the SG analysis, and the small blue circles show the positions of the galaxies belonging to structures in front of the cluster from the SG analysis. The red numbers in the middle right panel show the positions of the galaxies belonging to substructures in the cluster from the SG analysis, the number being the one given in Table 2. Finally, blue contours are the X-ray residuals, starting at the $2.5 \sigma$ level and spaced by $1 \sigma$ intervals. Lower panel: redshift histogram for CL J0152.7-1357. 



Fig. A.3. XMM-Newton X-ray image (upper left), residual image (upper right), and zoom on the residual image (lower left). The residual image was obtained by subtracting the model from the image for MS 0302.5+1717. The large blue circle in the upper left panel corresponds to a $500 \mathrm{kpc}$ radius circle centred on the literature cluster position. Finally, blue contours (present in some of the following figures) in the lower left panel are the X-ray residuals, starting at the $2.5 \sigma$ level and spaced by $1 \sigma$ intervals.

detected in the Chandra image and is indicated in Gilmour et al. (2009) as a point source.

\section{A.9. MACS J0744.9+3927 $\left(116.21583^{\circ},+39.4592^{\circ}\right.$, $z=0.6860)$}

The X-ray image of MACS J0744.9+3927 (Voges et al. 1999) is smooth, but the residuals show two significant sources (Fig. A.9). One is identified with an active galaxy (Gilmour et al. 2009) and the other one appears extended and seems to correspond spatially to the SZ excess detected by Korngut et al. (2011) and attributed to a region shocked by a merger. NED only provides two redshifts in the cluster field of view, so we cannot be sure we are really dealing with a substructure attached to the MACS J0744.9+3927 cluster. However, it is tempting to assume that we have detected a group falling onto the considered cluster, and we assume this in the present work.

\section{A.10. $R X$ J0847.1+3449 $\left(131.79708^{\circ},+34.8211^{\circ}\right.$, $z=0.5600)$}

The X-ray emission of RX J0847.1+3449 (Vikhlinin et al. 1998) is very faint (Fig. A.10). Subtracting a $\beta$-model basically only makes a point source appear south-west of the cluster, identified as an AGN in Vizier. NED provides a single redshift in the cluster area.

\section{A.11. MACS J0913 $\left(138.40277^{\circ},+40.94315^{\circ}, z=0.4420\right)$}

Although the X-ray emission appears quite smooth, a model subtraction reveals the presence of a structure very close to the cluster centre, perhaps due to an imperfect $\beta$-model subtraction. Such an effect is also seen in some of our simulations. We perhaps detect a very weak structure (significant only at the $2.5 \sigma$ level) east of the centre (Fig. A.11). The X-ray emission extent of this source is, however, very small, making it doubtful. This is confirmed by the Chandra data, pointing towards a very peaked core without any visible substructures.

Only a few redshifts are available in the cluster area, making any SG analysis impossible.

\section{A.12. Abell $851\left(145.73601^{\circ},+46.9894^{\circ}, z=0.4069\right)$}

The overall aspect of the $\mathrm{X}$-ray image is very clumpy, suggesting that one or several cluster mergers are taking place (Fig. A.12). The model-subtracted XMM-Newton X-ray image shows four 



Fig. A.4. Same as Fig. A.1 for XDCS cm J032903.1+025640 with straight blue lines (upper right) showing the interchip detector gaps.

main peaks that are more significant than the $3 \sigma$ level: a large and rather faint apparently extended source to the south-west, and three other more compact sources to the south, north, and east (Fig. A.12). Our residual image agrees qualitatively with that of De Filippis et al. (2003), who show that this cluster is most probably formed by the merger of two clusters of comparable masses. However, that the X-ray luminosity of the substructure is only about $10 \%$ that of the cluster implies that this is not a major merger. The comparison with spectroscopically confirmed active objects allows us to identify the east compact source as an AGN. We have no Chandra image to characterize the other two compact sources, but given their shape in the XMM-Newton data, it is likely that they are also AGNs.

A large number of redshifts are available in NED, with 213 galaxies in the $[0.39,0.42]$ redshift range. The velocity histogram around $z \sim 0.41$ is clearly asymmetric, and the SG analysis shows the presence of several substructures. In particular, the most massive (group SG3 in Table 2) is clearly associated with the extended X-ray source, all the galaxies belonging to this group being located inside the X-ray contours. Only two galaxies that are not members of Abell 851 and are included in galaxy structures along the line of sight are located inside the considered X-ray contours. This makes projection effects very unlikely.

\section{A.13. MS $1054-03\left(164.25093^{\circ},-3.6243^{\circ}, z=0.8231\right)$}

The model-subtracted XMM-Newton image of MS 1054-03 shows three main peaks above the $5.5 \sigma$ level: a large and rather faint apparently extended source to the west (too far from the cluster centre to be due to a $\beta$-model subtraction that is not perfect), and two other more compact and somewhat brighter sources to the south and north east (Fig. A.13). This agrees with the statement by Gioia et al. (2004), who classify MS 1054-03 as a "young, massive, highly luminous cluster with significant substructure".

The comparison with the Chandra image clearly shows that the two compact sources are probably due to point sources even though none of them corresponds to spectroscopically known active objets. The XMM-Newton extended residual to the west is also extended in the Chandra image, so we can probably conclude that we are dealing with extended thermal emission related to the cluster: a real substructure in the MS 1054-03 cluster of galaxies.

On the optical side, the redshift histogram shows a broad distribution for the 326 redshifts in the [0.81, 0.85] range (Fig. A.13). The SG analysis shows the presence of a massive substructure of 26 galaxies (group SG2), which can probably be considered as the main core of MS 1054-03. We also detect three other less populated substructures (Table 2). Among them, 



Fig. A.5. Same as Fig. A.3 for RX J0337.6-2522.

the SG1 group is the most massive and is probably associated with the X-ray substructure described above.

\section{A.14. UM 425 cluster $\left(170.83542^{\circ},+1.6294^{\circ}, z=0.7685\right)$}

The UM 425 cluster is located along the line of sight of a known quasar pair. It was first supposed to be at the same redshift than the quasars $(z \sim$ 1.47: Mathur \& Williams 2003). Later, Green et al. (2005), proposed a redshift of $\sim 0.77$ based on VLT spectroscopy.

The UM 425 candidate cluster shows no obvious substructures in X-rays and is well fit by a simple model (Fig. A.14) without any significant X-ray residual. The redshift histogram of the UM 425 Cluster shows a peak at $z \sim 0.7685$ (the redshift given by NED), in agreement with Green et al. (2005), and a smaller background peak at $z \sim 0.87$ (Fig. A.14). However, the SG analysis does not detect any structure (cluster or subcluster). Even if there are only eight galaxies in the [0.760, 0.773] range, this leads to doubts about the massive cluster nature of the UM 425 cluster. We are perhaps intercepting a filament (explaining the peak in the redshift histogram), including a quasar emitting in X-rays.

\section{A.15. MS 1137.5+6624 $\left(175.09696^{\circ},+66.1449^{\circ}, z=0.7820\right)$}

The residual X-ray image of MS $1137.5+6624$ shows two compact sources to the north-east and the south-west (Fig. A.15), both identified with active objects on the line of sight (Gilmour et al. 2009). Some excess diffuse emission is also left after subtracting the $\beta$-model, exactly at the cluster location. This is probably because a $\beta$-model is not able to reproduce the strong peak at the cluster centre visible in the Chandra image (Fig. A.15). We therefore consider that we are not dealing with a real X-ray substructure, as also suggested by the Chandra X-ray image of Maughan et al. (2008).

On the optical side, the velocity histogram is asymmetric. There are 17 redshifts in the cluster range (Fig. A.15), but the SG does not detect any substructure in the cluster. Since no substructure appears in the SG analysis or in X-rays, we are probably dealing with a fairly relaxed structure.

\section{A.16. CLG J1205+4429 $\left(181.46410^{\circ},+44.4860^{\circ}\right.$, $z=0.5915)$}

The model-subtracted XMM-Newton X-ray image of CLG J1205+4429 shows three main peaks (in addition to the detector interchip residuals shown in Fig. A.16). Two of them are compact sources north-east of the cluster (identified as AGNs in Gilmour et al. 2009), and the comparison with the Chandra image clearly shows that these sources are point-like. The third X-ray source in the X-ray residual image seems extended and is roughly centred on the cluster position.

On the optical side, the redshift histogram shows that the line of sight to CLG J1205+4429 intercepts several structures 




Fig. A.6. Same as Fig. A.2 for MACS J0454.1-0300.

(Fig. A.16). A peak is apparent at $z=0.5915$ (the value given by NED) in the redshift histogram, with 11 galaxies in the [0.582, 0.600 ] range. The SG method interprets these galaxies as a single cluster without any substructure.

It is therefore likely that the X-ray residual corresponding to the third X-ray source is not a classical substructure (a major infalling group made of relatively bright galaxies), but it could possibly be part of the central cluster X-ray emission incorrectly removed by the subtraction of a $\beta$-model fit. However it is too extended to be just a wrong $\beta$-model subtraction of the cluster central emission and CLG J1205+4429 is not a cool-corelike cluster (Ulmer et al. 2005). This structure instead resembles what was observed in Coma by Neumann et al. (2003), where relatively diffuse X-ray emission is associated to both NGC 4874 and NGC 4889, in addition to the global cluster $\beta$-model emission. This, together with the fact that CLG J1205+4429 is a structure with properties close to those of a fossil group (Ulmer et al. 2005), speaks in favour of an old group with its own 


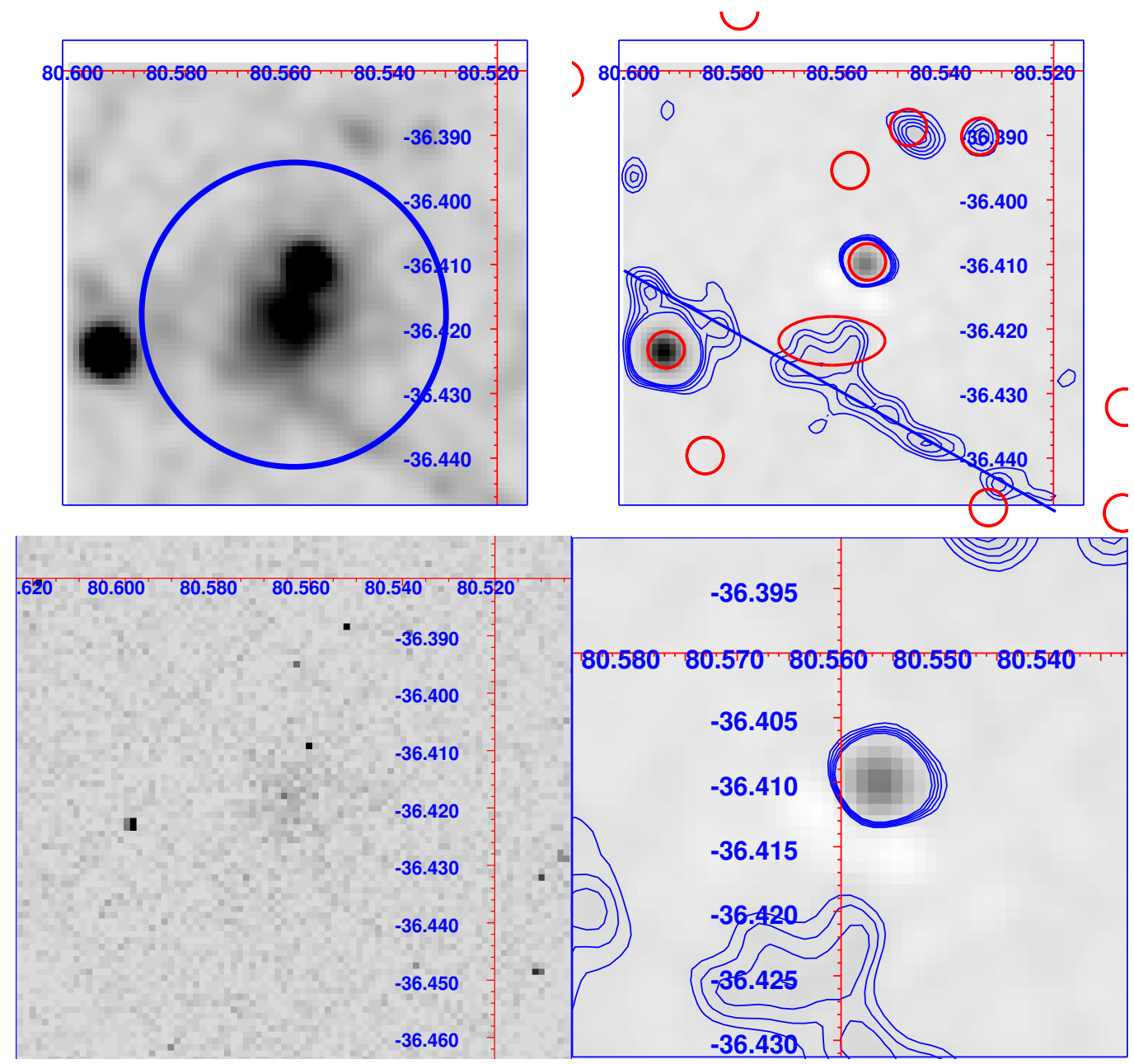

Fig. A.7. XMM-Newton X-ray image (upper left), residual XMM-Newton image (upper right), Chandra image (lower left), and zoom on the residual XMM-Newton image (lower right). The residual image was obtained by subtracting the model from the XMM-Newton image for BMWHRI J052215.8-362452. The large blue circle in the upper left panel corresponds to a $500 \mathrm{kpc}$ radius circle centred on the literature cluster position. Finally, blue contours are the X-ray residuals, starting at the $2.5 \sigma$ level and spaced by $1 \sigma$ intervals.

original X-ray halo (seen as the central X-ray residual) which acted as a primary seed to accrete more matter (without experiencing major merging events) and is at the origin of the underlying X-ray $\beta$-model.

\section{A.17. RXC J1206.2-0848 $\left(181.54991^{\circ},-8.8000^{\circ}\right.$ $z=0.4400)$}

The X-ray emission is quite smooth. No point sources are detected in the Chandra image. The subtraction of a model shows the existence of two small excesses, one to the south-east and one at the cluster centre (Fig. A.17). The cluster-centred excess is probably due to the inability to correctly reproduce the cluster central surface brightness profile with a $\beta$-model, which is quite peaked. For this cluster, we have enough counts in the centre to measure a central temperature. Limiting our analysis to a $56 \mathrm{kpc}$ radius, we obtain $6.00 \pm 0.75 \mathrm{keV}$, compared to $9.36 \pm 0.55 \mathrm{keV}$ for the whole cluster. This indicates the possible presence of a cool core that could explain the central X-ray excess.

The redshift histogram of RXC J1206.2-0848 shows a broad double peak around $z \sim 0.44$ (Fig. A.17), and looks quite asymmetric. There are 53 galaxies in the $[0.42,0.46]$ range. The SG analysis shows the presence of four rather small substructures with three to five galaxies (see Table 2). SG4 could possibly be associated with the small south-east X-ray residual.

$$
\begin{aligned}
& \text { A.18. LCDCS } 0504=C l 1216.8-1201\left(184.18845^{\circ},\right. \\
& \left.-12.02147^{\circ}\left(184.18792^{\circ},-12.02139^{\circ}\right), z=0.7943\right)
\end{aligned}
$$

The X-ray image of LCDCS 0504 shows a double structure (Fig. A.18) after $\beta$-model subtraction. The stronger source to the west is likely to be a point-like source superimposed on the line of sight (there is no Chandra image for this cluster, and no known AGN along the line of sight).

We also have three other $\geq 3 \sigma$ significant $\mathrm{X}$-ray sources in the residual map. None is associated with a galaxy structure detected by the SG method on the line of sight. Since they are not close to the cluster, they do not affect the substructure analysis.

On the optical side, there are 65 galaxies with redshifts in the [0.78, 0.81] range (Fig. A.18). The characteristics of the seven substructures found by the SG method are given in Table 2. The eastern X-ray peak can be associated with SG1 or SG4, but given the SG masses listed in Table 2, we choose to associate the X-ray source with SG1 (because in view of the relative masses of SG1 and SG4, SG4 would not be detected in X-rays). This small group would therefore be located $\sim 5300 \mathrm{~km} \mathrm{~s}^{-1}$ beyond the main cluster. 



Fig. A.8. Same as Fig. A.7 for MACS J0647.7+7015.

A more detailed analysis of this cluster will be presented elsewhere (Guennou et al. 2013).

\section{A. 19. BMW-HRI J122657.3+333253 (186.74167 , 33.5484 , $z=0.8900)$}

BMW-HRI J122657.3+333253 was discovered in X-rays by Ebeling et al. (2001b). Its residual X-ray image shows several compact sources, all known as AGN (Gilmour et al. 2009), and most of them being visible as point sources in the Chandra image. In addition, we detect a more extended source, directly south of the cluster itself, which is not correlated with any known structure along the line of sight. Jee \& Tyson (2009) made a weak lensing mass reconstruction of this cluster and also found a subclump about 40 arcsec south-west of the cluster centre. This position corresponds to a temperature enhancement reported by Maughan et al. (2007). It is consistent with features detected in SZ with MUSTANG by Korngut et al. (2011), who interpret this cluster in a merger scenario where a small cluster has crossed a larger one on a trajectory oriented towards the south-west.

Even if it is not straightforward to assign this excess X-ray emission to one of the SG detected substructures, it is likely that group SG6 (see Table 2) is the optical manifestation of this emission. However, this group is too sparsely sampled (only
3 redshifts) to provide a velocity dispersion. Its mean redshift puts it $\sim 4600 \mathrm{~km} \mathrm{~s}^{-1}$ beyond the cluster's main core (SG1).

\section{A.20. $\mathrm{GHO} 1322+3027\left(201.20091^{\circ},+30.1928^{\circ}, z=0.7550\right)$}

The residual X-ray image along the GHO 1322+3027 line of sight shows several X-ray sources (Fig. A.20) that are more significant than the $2.5 \sigma$ level. Part of them are explained by the MOS1 interchip gap separation (Fig. A.20). There is a pointlike X-ray source directly south of the cluster. This source is not known as an AGN in NED or Vizier, and without Chandra images available, we cannot be $100 \%$ sure that we are really dealing with an AGN. We also detect three poorly significant X-ray sources north of the cluster that are all very well correlated with galaxies belonging to foreground galaxy structures on the line of sight. Finally, we detect in the residual X-ray image a very significant X-ray emission superimposed on the cluster centre. It is very likely that we are dealing with an imperfect $\beta$-model subtraction, but without Chandra data, it is difficult to conclude whether it is an active galaxy in the middle of the cluster (the $\mathrm{cD}$ ?) or if this cluster has a cool-core.

The redshift histogram is rather asymmetric (Fig. A.20) with 38 galaxies in the $[0.745,0.775]$ redshift range. However the SG analysis detects a single massive structure: the cluster 

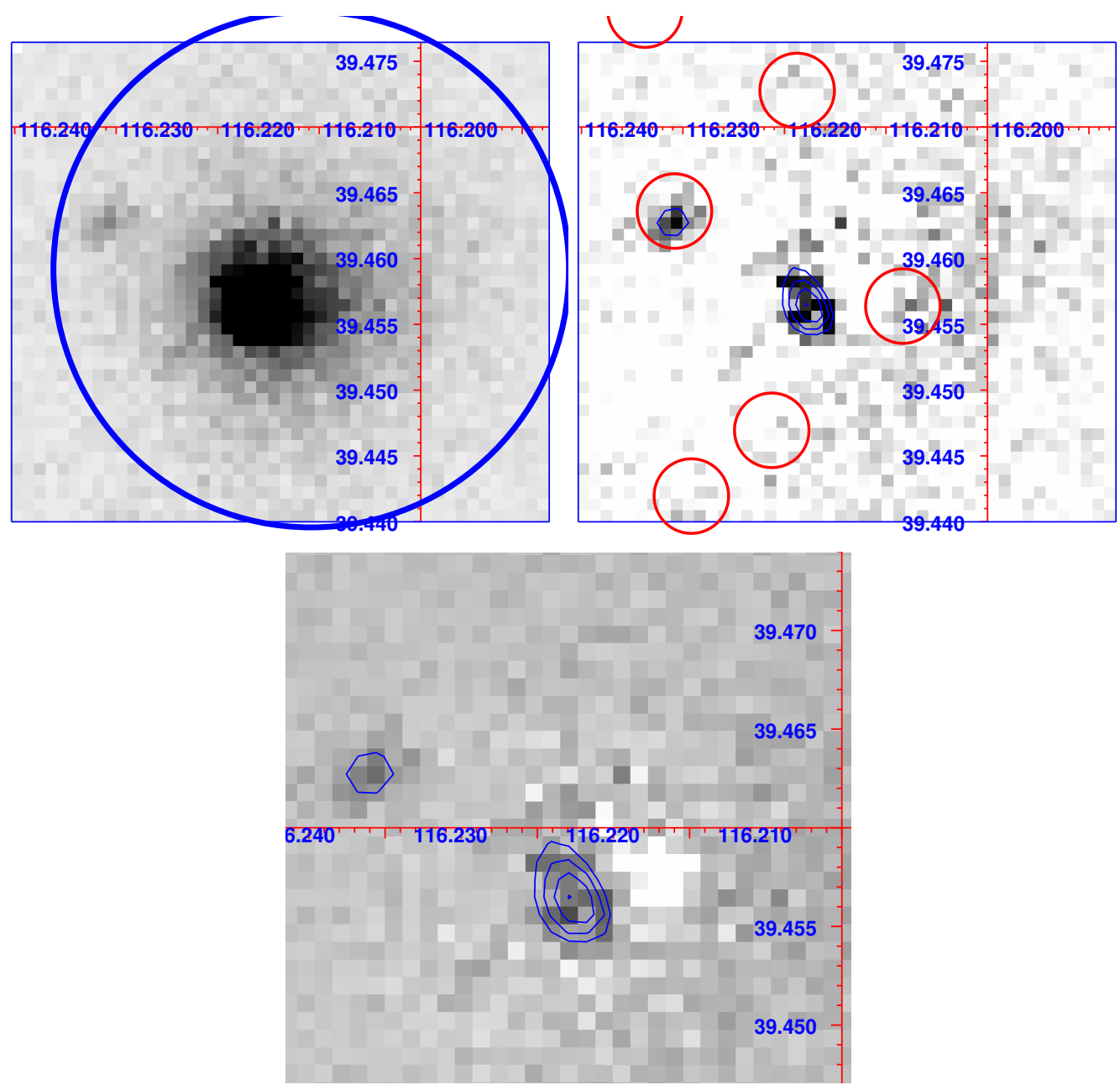

Fig. A.9. Same as Fig. A.3 for MACS J0744.9+3927.

itself. GHO $1322+3027$ therefore does not exhibit significant substructures.

\section{A.21. $\mathrm{ZwCl} 1332.8+5043\left(203.58333^{\circ},+50.5151^{\circ}\right.$, $z=0.6200)$}

The X-ray image of $\mathrm{ZwCl} 1332.8+5043$ shows a diffuse source, and two compact sources to the north-west and north-east (Fig. A.21). After model subtraction, only these two sources are left. The Chandra image confirms that we are dealing with a double point source to the north-west (known as two active galaxies in Gilmour et al. 2009) and a single point source to the north-east (also present in Gilmour et al. 2009). So we detect no substructures in this cluster.

NED provides a single galaxy redshift, so the SG analysis is impossible.

\section{A.22. $L C D C S 0829=R X J 1347.5-1145(206.88333$, $\left.-11.7617^{\circ}, z=0.4510\right)$}

LCDCS 0829, also known as RXJ1347.5-1145, is a very hot and luminous $\mathrm{X}$-ray cluster known to show several gravitational arcs (Schindler et al. 1995). The XMM-Newton image of LCDCS 0829 is indeed bright (Fig. A.22), and the subtraction of a $\beta$-model shows excess emission south and north of the cluster. The northern part is probably the superposition of diffuse emission and of a point source (visible in the Chandra image). The southern excess roughly corresponds spatially to the emission detected in SZ by Komatsu et al. (2001), as later confirmed with better data by Korngut et al. (2011). These two diffuse sources suggest there is a recent merger (Korngut et al. 2011 and references therein). We also see a small deficit of emission just north of the cluster centre.

On the optical side, the redshift histogram is somewhat asymmetric (Fig. A.22), with 50 galaxies with redshifts in the $[0.425,0.47]$ range. The SG method first detects a large group of 50 galaxies (not listed in Table 2) at $z \sim 0.45$ with an estimated mass of $3.83 \times 10^{14} M_{\odot}$. This group is then split into four smaller substructures (see Table 2). Among them, SG1 seems associated with the northern extended emission, while SG4 seems associated with the southern extended emission.

\section{A.23. $\operatorname{LCDCS} 0853\left(208.53958^{\circ},-12.5164^{\circ}, z=0.7627\right)$}

The X-ray image of LCDCS 0853 shows emission at the cluster position (Fig. A.23), which is fully accounted for by a simple model, besides a very minor residual close to the cluster centre (only significant at the $2.5 \sigma$ level and extended over less than 3 arcsec). We also see the residuals of the PN interchip separation.

The full redshift histogram up to $z=1$ shows that the line of sight to the cluster is intercepting several structures (Fig. A.23). There are 18 galaxies with redshifts in the [0.75, 0.77] cluster range. The SG method detects a main structure (see Table 2), but 


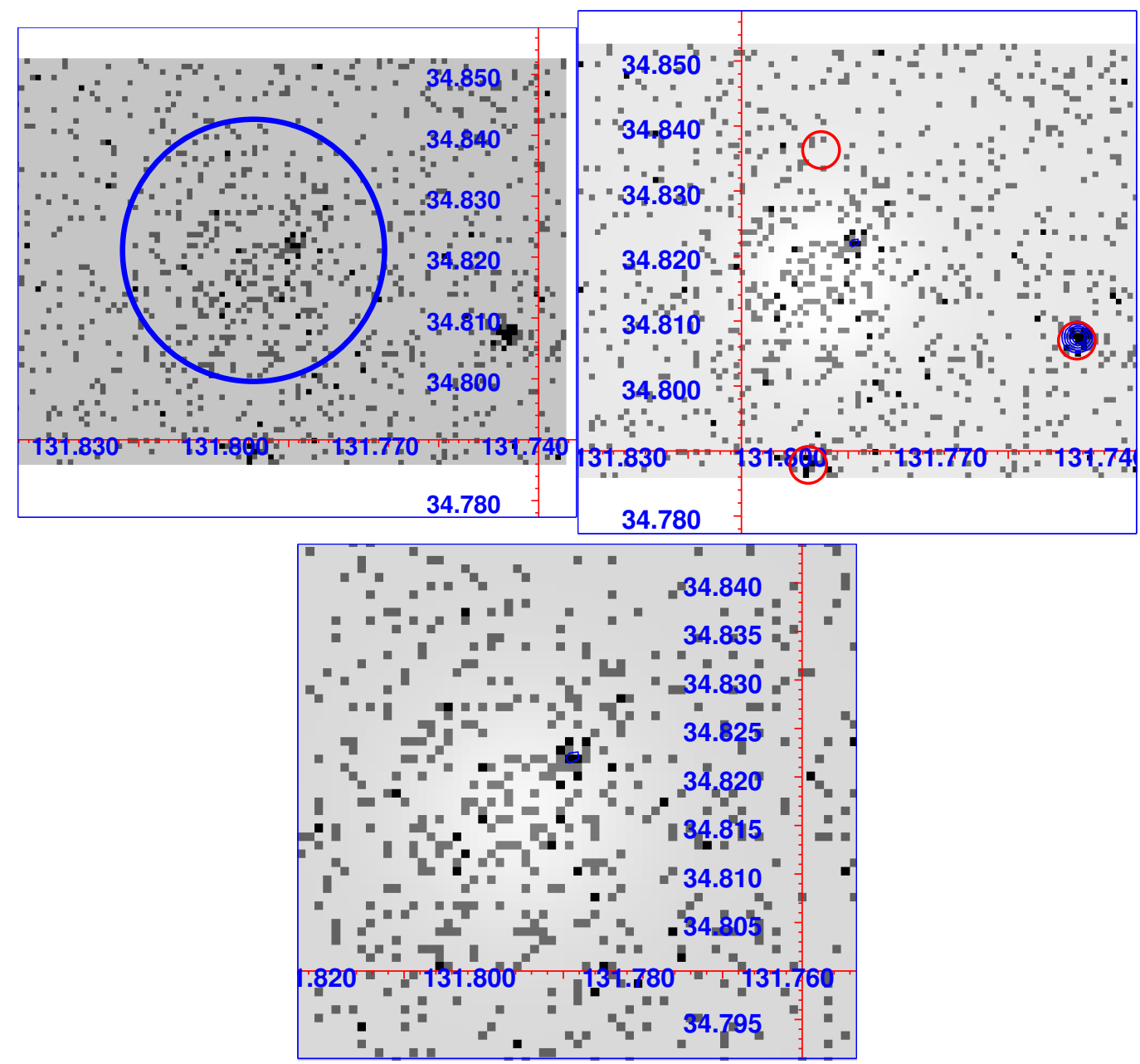

Fig. A.10. Same as Fig. A.3 for RX J0847.1+3449.

we must note that our spectroscopic catalogue does not cover the full field of view.

\section{A.24. $R X J 1354.2-0221\left(208.57042^{\circ},-2.3631^{\circ}, z=0.5460\right)$}

The X-ray image of RX J1354.2-0221 (Vikhlinin et al. 1998) shows a very weak extended source (Fig. A.24), barely visible in the Chandra image, and no residuals appear after subtracting a model. Several point sources appear in the Chandra image, most of them known in Gilmour et al. (2009). NED gives a redshift $z=0.5460$ for the cluster, but only two redshifts are available in this range, preventing from any SG analysis.

\section{A.25. MACS J1423.8+2404 (215.95125,$+24.0797^{\circ}$, $z=0.5450)$}

This is the only cluster for which there are no XMM-Newton data, but for which the Chandra image is deep enough to allow us to remove a $\beta$-model from the cluster image (Fig. A.25), so we include it in this section. The $\mathrm{X}$-ray emission is strong and slightly elongated. After subtracting a $\beta$-model, we see several low significance X-ray residuals north east of the cluster. We also detect two brighter sources close to the cluster centre, respectively located close to the cluster centre and toward the south-west. The brighter one (close to the cluster centre) is probably due to the fact that the $\beta$-model subtraction is not perfect, while the other one is quite extended.
We only have nine galaxy redshifts in the [0.536, 0.552] range (Fig. A.25), and the SG method only detects the cluster as a three-galaxy structure, so we can only suggest that the extended X-ray source south-west of the cluster is a substructure of the MACS J1423.8+2404 cluster.

\section{A.26. $\mathrm{GHO} 1602+4312\left(241.10483^{\circ},+43.0813^{\circ}, z=0.8950\right)$}

The X-ray image of GHO 1602+4312 shows a faint elongated structure. The residual image shows some emission due to the PN interchip separation (at the bottom of upper right of Fig. A.26) and several barely significant ( $2.5 \sigma$ level) X-ray sources. Two of these sources are located at the edge of the elongated X-ray cluster emission. They may be due to structures on the line of sight (see upper right Fig. A.26), as suggested by the redshift histogram showing the presence of several small structures on the line of sight (Fig. A.26). These two faint X-ray sources also could be minor infalling groups that are not detected by the SG analysis (see below). There are no X-ray point sources detected in the field of view, neither by Gilmour (2009) or in Chandra data.

Only the cluster itself is detected by the SG analysis (group SG1 in Table 2). The redshift histogram of the cluster shows a rather symmetric peak at $z \sim 0.89$ with 26 galaxies in the [0.88, 0.92] range, out of which five form the blue wing of the redshift histogram. This cluster is part of a supercluster, according to Gal et al. (2008). 

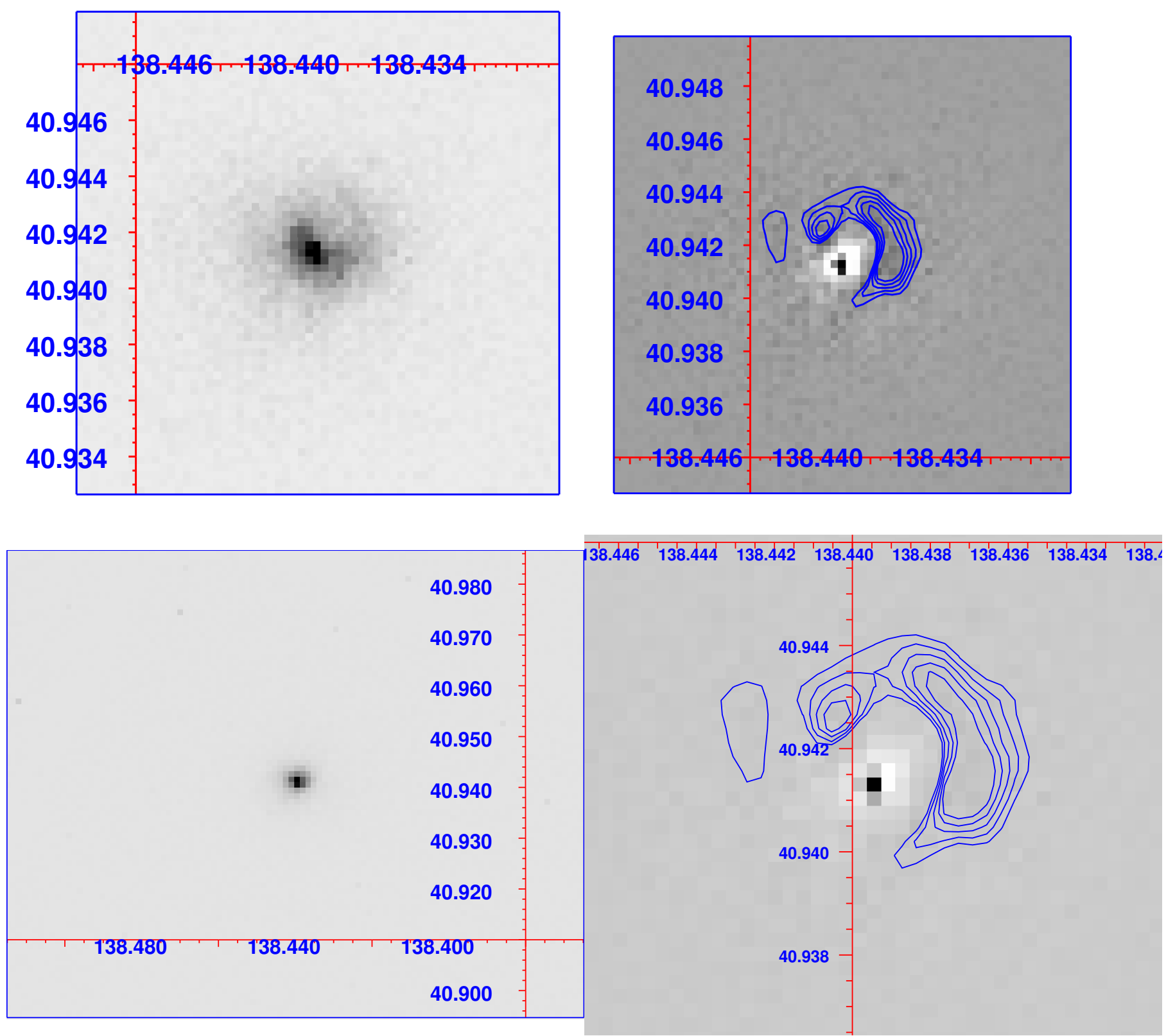

Fig. A.11. Same as Fig. A.7 for MACS J0913.

\section{A.27. MS $1621.5+2640\left(245.89863^{\circ},+26.5638^{\circ}, z=0.4260\right)$}

The XMM-Newton exposure is short $(2.2 \mathrm{ksec})$, but though the image is quite faint, it shows the cluster core (Fig. A.27). We detect in the residual map a compact X-ray source south-east of MS 1621.5+2640 identified as an active object by Gilmour et al. (2009) and also visible in the Chandra image.

The redshift histogram of MS 1621.5+2640 shows a strong and somewhat asymmetric peak at $z \sim 0.427$ (Fig. A.27), with 104 galaxies in the $[0.412,0.448]$ range. The SG method detects a main structure and three smaller substructures (see Table 4). Given the very short XMM-Newton exposure time, it is impossible to detect them in X-rays, even if some of them seem massive from the $\mathrm{SG}$ analysis.
A.28. MS 2053.7-0449 (314.09321 $\left.,-4.62873^{\circ}, z=0.5830\right)$ and CXOU J205617.1-044155 = CXOSEXSI J205617.1-044155 $\left(314.07150^{\circ},-4.69864^{\circ}, z=0.6002\right)$

MS 2053.7-0449 (Harrison et al. 2003) is a fairly bright cluster, probably physically associated with the smaller cluster CXOSEXSI J205617.1-044155. The X-ray residual image does not show any substructures or point sources more significant than the $2.5 \sigma$ level (Fig. A.28). Gilmour et al. (2009) detected several AGN, but they are probably too faint to appear as significant sources. This statement is in good agreement with the Chandra image not showing any bright point source.

On the optical side, the redshift histogram in an area of 2.2 arcmin radius around MS 2053.7-0449 is shown in Fig. A.28. There are 30 galaxies in the $[0.57,0.60]$ redshift range, and the 

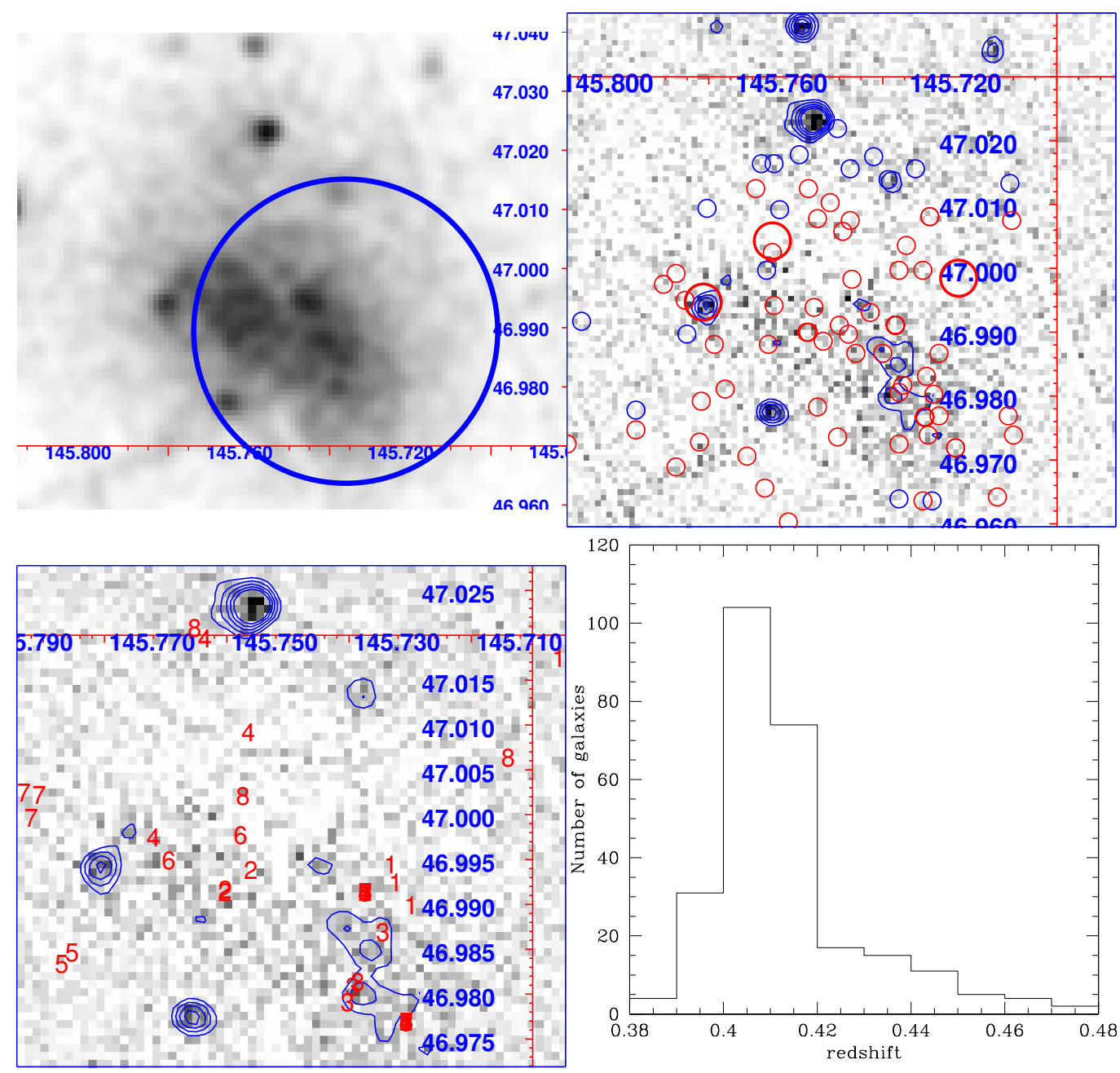

Fig. A.12. Same as Fig. A.1 for Abell 851.

SG method finds a single massive structure (see Table 2) associated with cluster MS 2053.7-0449 itself. MS 2053.7-0449 is therefore a cluster without any detectable substructures, whether in X-rays or in the optical. It may merge with CXOSEXSI J205617.1-044155 in the future.

As seen in Fig. A.29, CXOSEXSI J205617.1-044155 (Harrison et al. 2003) is rather faint in X-rays (X-ray luminosity of $4.37 \times 10^{43} \mathrm{erg} / \mathrm{s}$ ) and not visible in the Chandra image. The $\mathrm{X}$-ray image may show an X-ray point source north-west of the cluster also listed in Gilmour et al. (2009). This point source, as well as two other ones known in Gilmour et al. (2009), is visible in the Chandra image. The subtraction of a $\beta$-model on the XMM-Newton image does not allow detecting any significant X-ray residual. In a zone of 2.2 arcmin around CXOSEXSI $\mathrm{J} 205617.1-044155$, there is only one redshift $(z=0.6002)$ corresponding to the cluster dominant galaxy.

\section{A.29. $\mathrm{GHO} 2143+0408\left(326.52000^{\circ},+4.3886^{\circ}, z=0.5310\right)$}

This is a massive X-ray cluster discovered by Vikhlinin et al. (1998) and member of the WARPS survey (WARP J2146.0+0423, Perlman et al. 2002). The X-ray residual image does not show any $\mathrm{X}$-ray source more significant than the $2.5 \sigma$ level, and there is no known active object along the line of sight (Fig. A.30).
We have obtained 28 redshifts with the ESO VLT and FORS2 (see Table C.2 at the end of Appendix C). The SG method detects a few structures along the line of sight, but none in the cluster region. The cluster itself is detected by the SG method without any additional substructure at the same redshift.

A.30. $R X$ J2202.7-1902 (330.68708 $\left.{ }^{\circ},-19.0361^{\circ}, z=0.4380\right)$

The X-ray image of RX J2202.7-1902 (Vikhlinin et al. 1998) shows a very weak extended source (Fig. A.31). No significant residuals appear after subtracting a model. NED gives only a few redshifts along the cluster line of sight, preventing any SG analysis.

\section{A.31. $R X J 2328.8+1453\left(352.20792^{\circ},+14.8867^{\circ}\right.$, $z=0.4970)$}

The X-ray image of RX J2328.8+1453 (Vikhlinin et al. 1998) appears quite diffuse (Fig. A.32), and the subtraction of a model shows two small $2.5 \sigma$ level sources (too faint to provide a reliable X-ray luminosity). One of them is identified with an active object in Vizier.

NED gives for this cluster a redshift $z=0.4970$. There are only 5 other redshifts available in an area of 5 arcmin radius around this cluster, and no galaxy is at a similar redshift, so an $\mathrm{SG}$ analysis is impossible. 
L. Guennou et al.: Substructures in $0.4<z<0.9$ clusters



Fig. A.13. Same as Fig. A.2 for MS 1054-03. 



O
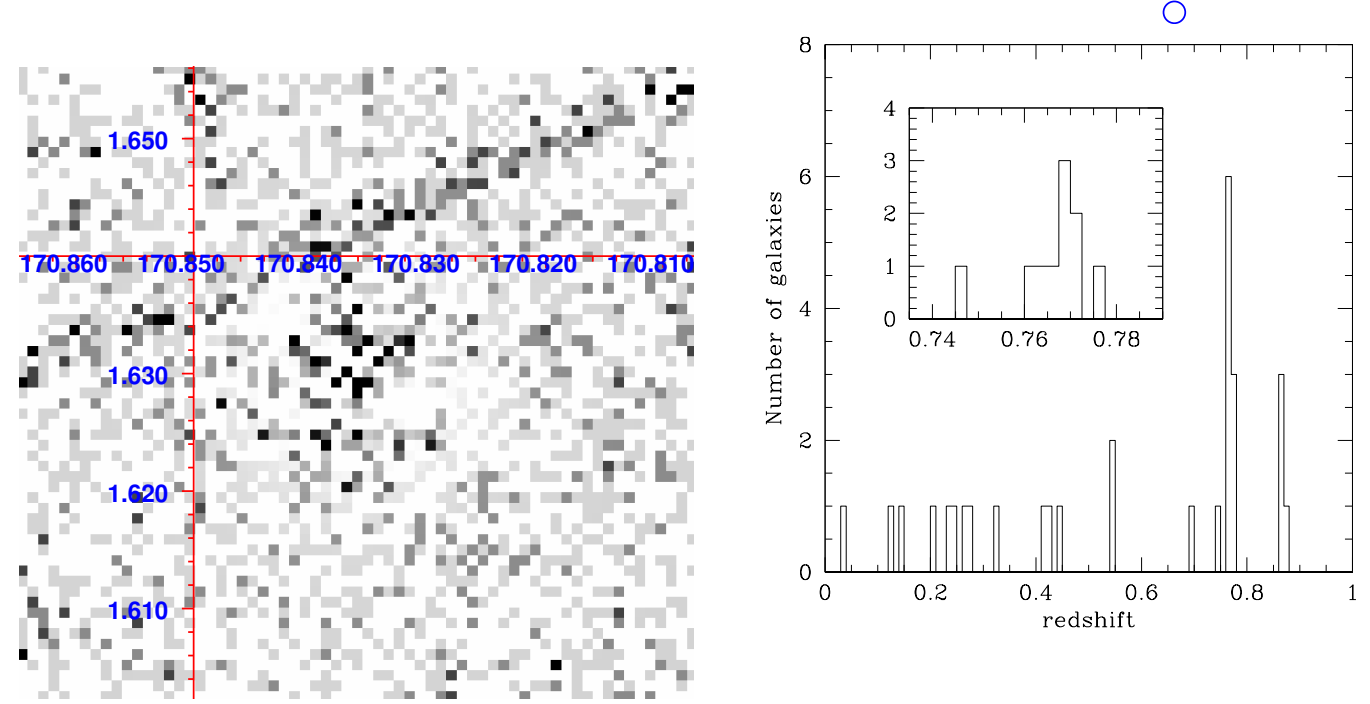

Fig. A.14. Same as Fig. A.1 for the UM 425 cluster. The insert in the redshift histogram (lower right) shows a zoom around the cluster redshift. 
L. Guennou et al.: Substructures in $0.4<z<0.9$ clusters


Fig. A.15. Same as Fig. A.2 for MS 1137.5+6624. 

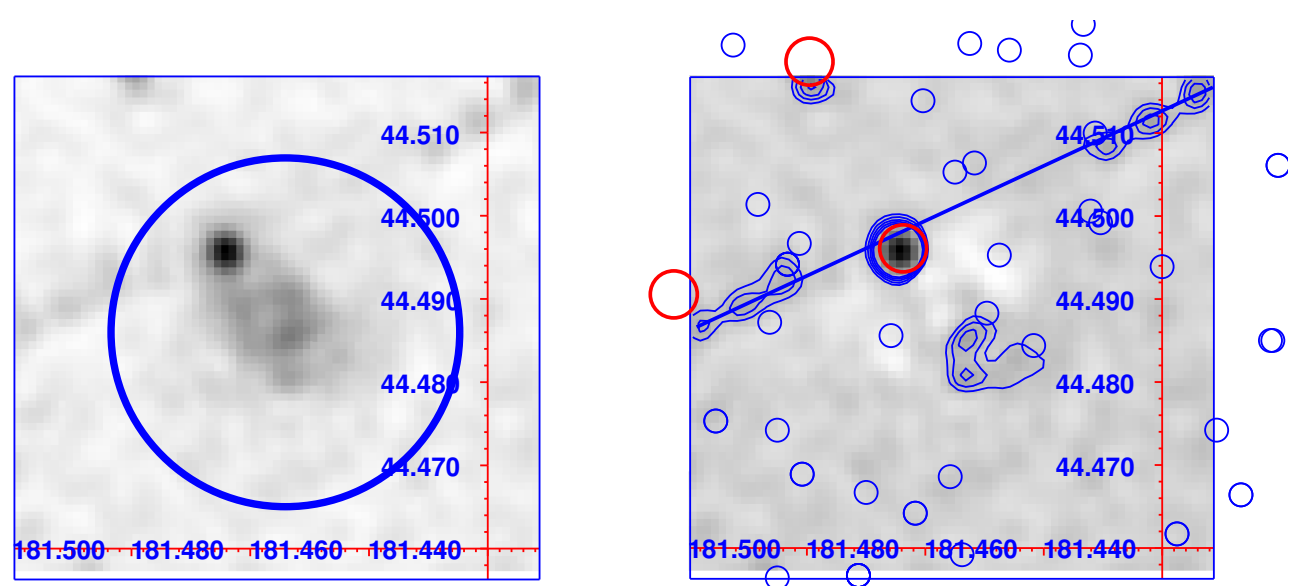

(Q) 0


Fig. A.16. Same as Fig. A.2 for CLG J1205+4429. 

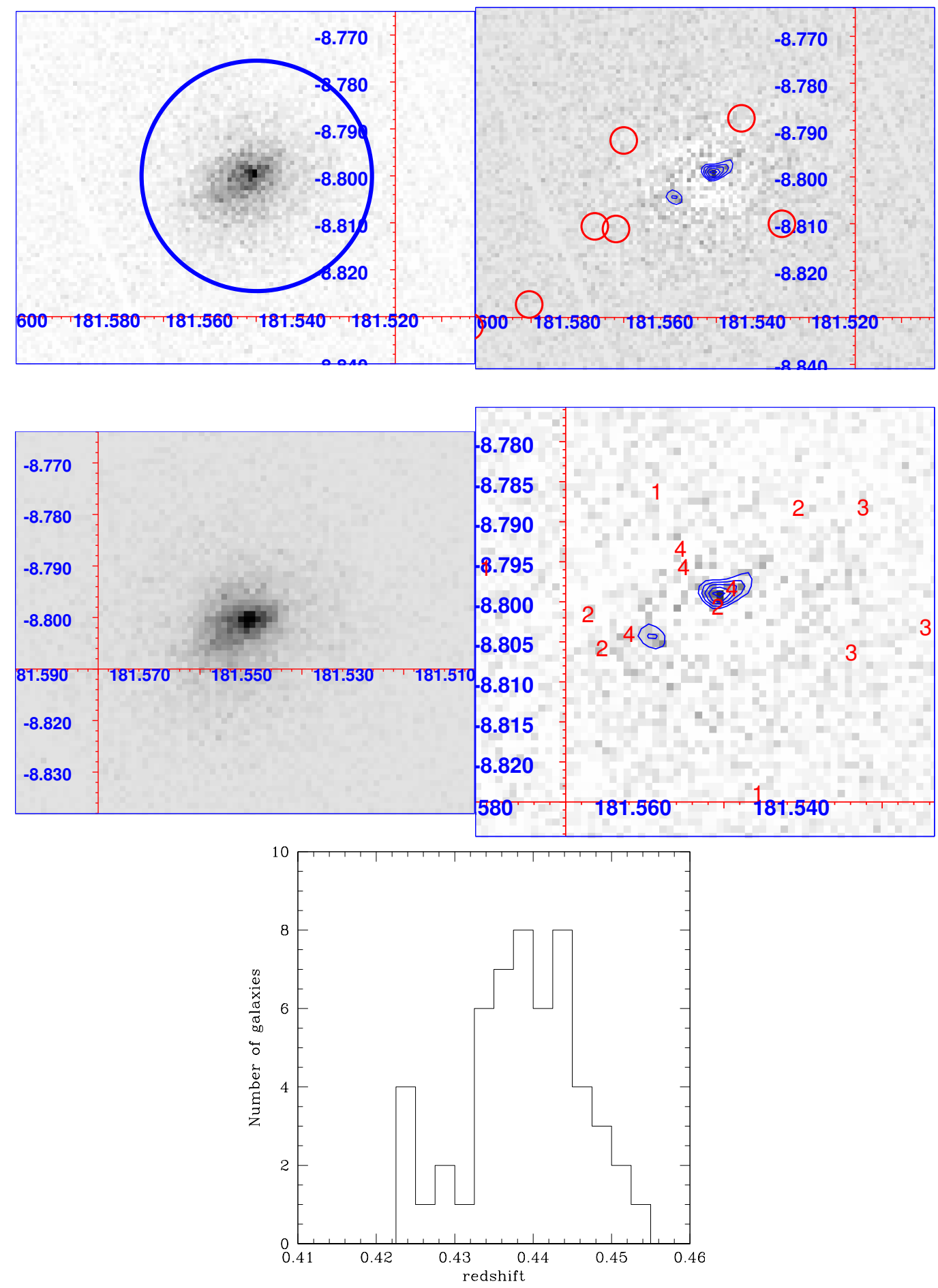

Fig. A.17. Same as Fig. A.2 for RXC J1206.2-0848. 
A\&A 561, A112 (2014)
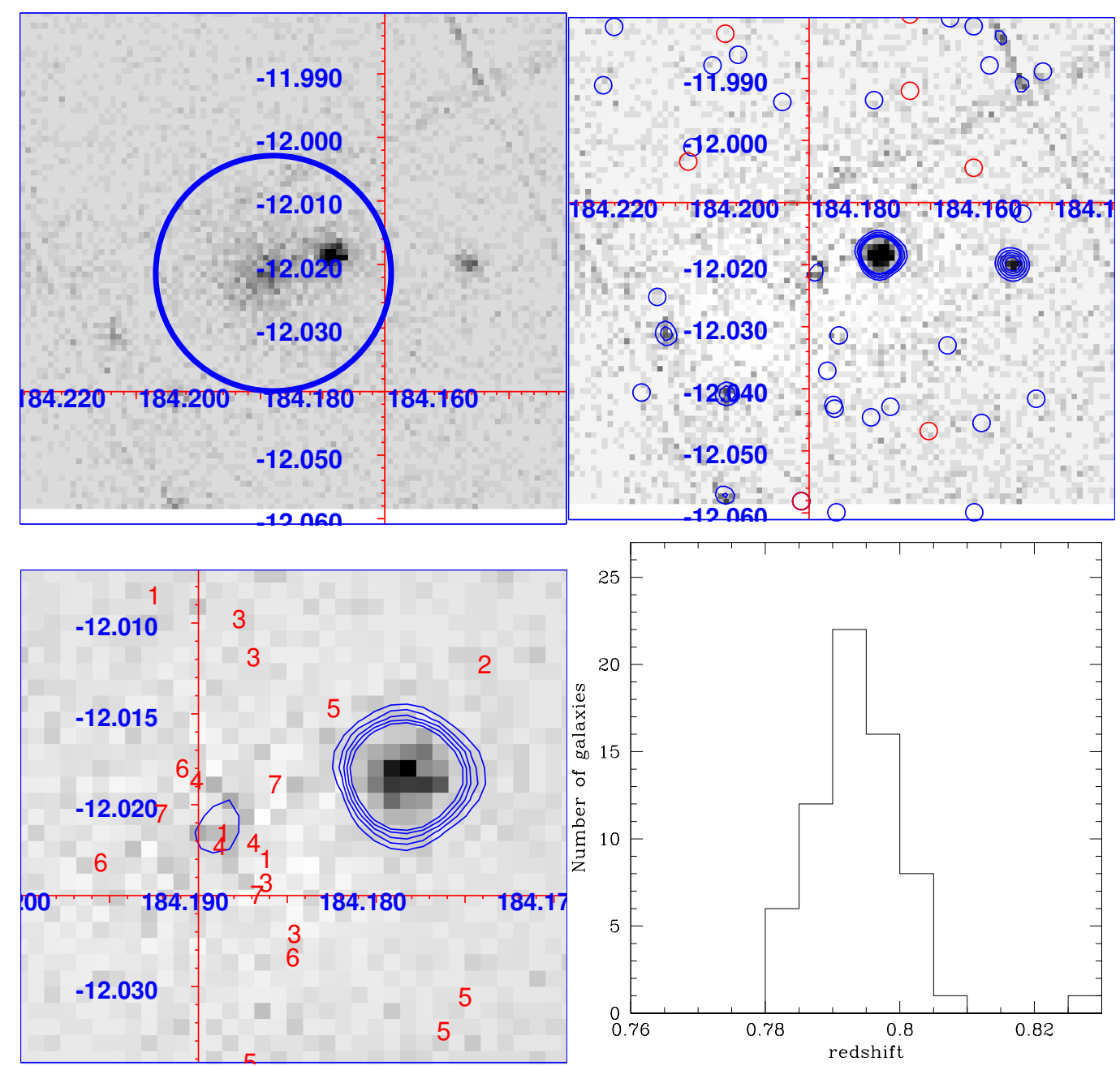

Fig. A.18. Same as Fig. A.1 for LCDCS 0504. 
L. Guennou et al.: Substructures in $0.4<z<0.9$ clusters

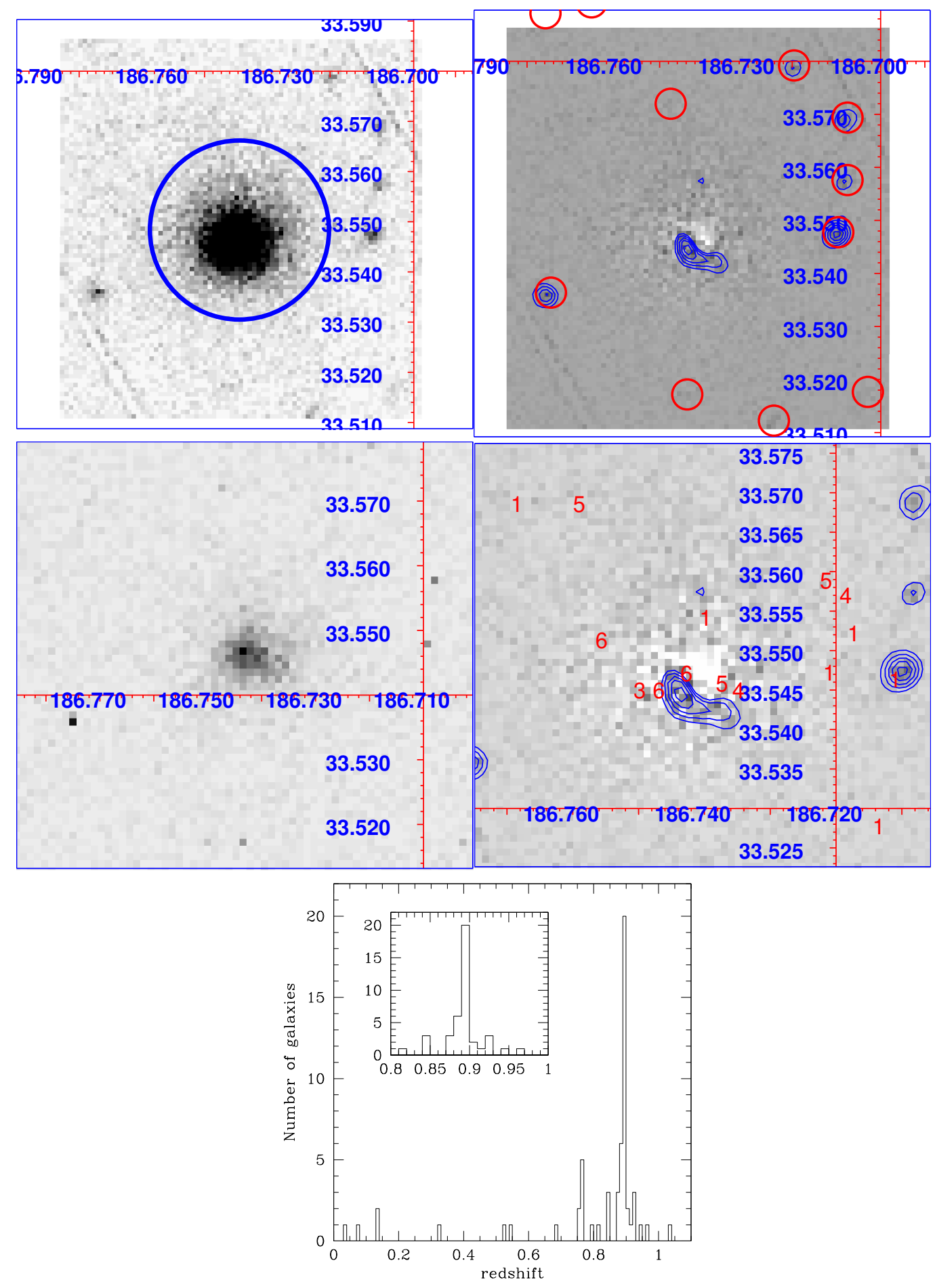

Fig. A.19. Same as Fig. A.2 for BMW-HRI J122657.3+333253. 
A\&A 561, A112 (2014)


Fig. A.20. Same as Fig. A.1 for GHO 1322+3027. 
L. Guennou et al.: Substructures in $0.4<z<0.9$ clusters
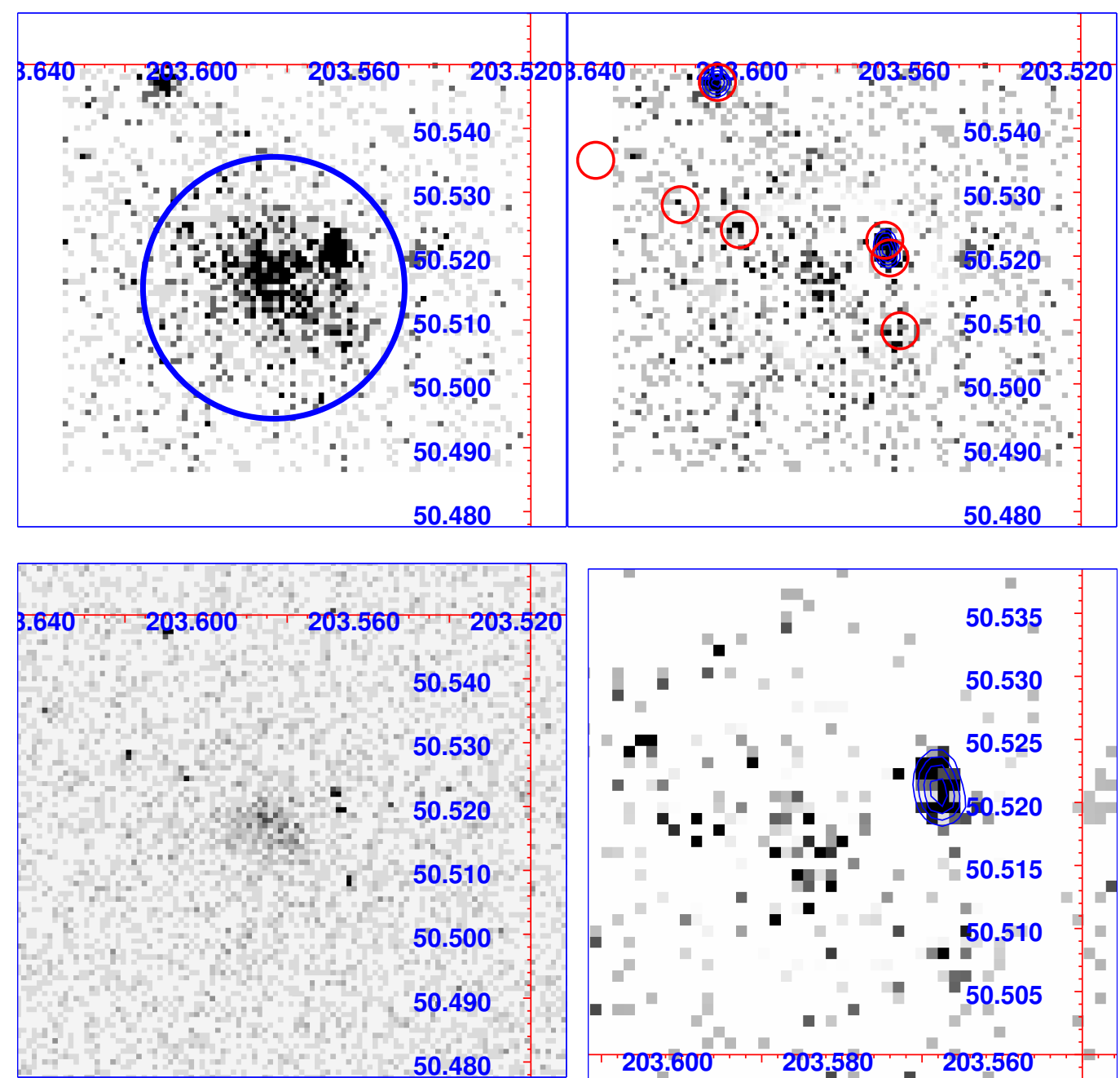

Fig. A.21. Same as Fig. A.7 for $\mathrm{ZwCl} 1332.8+5043$. 
A\&A 561, A112 (2014)

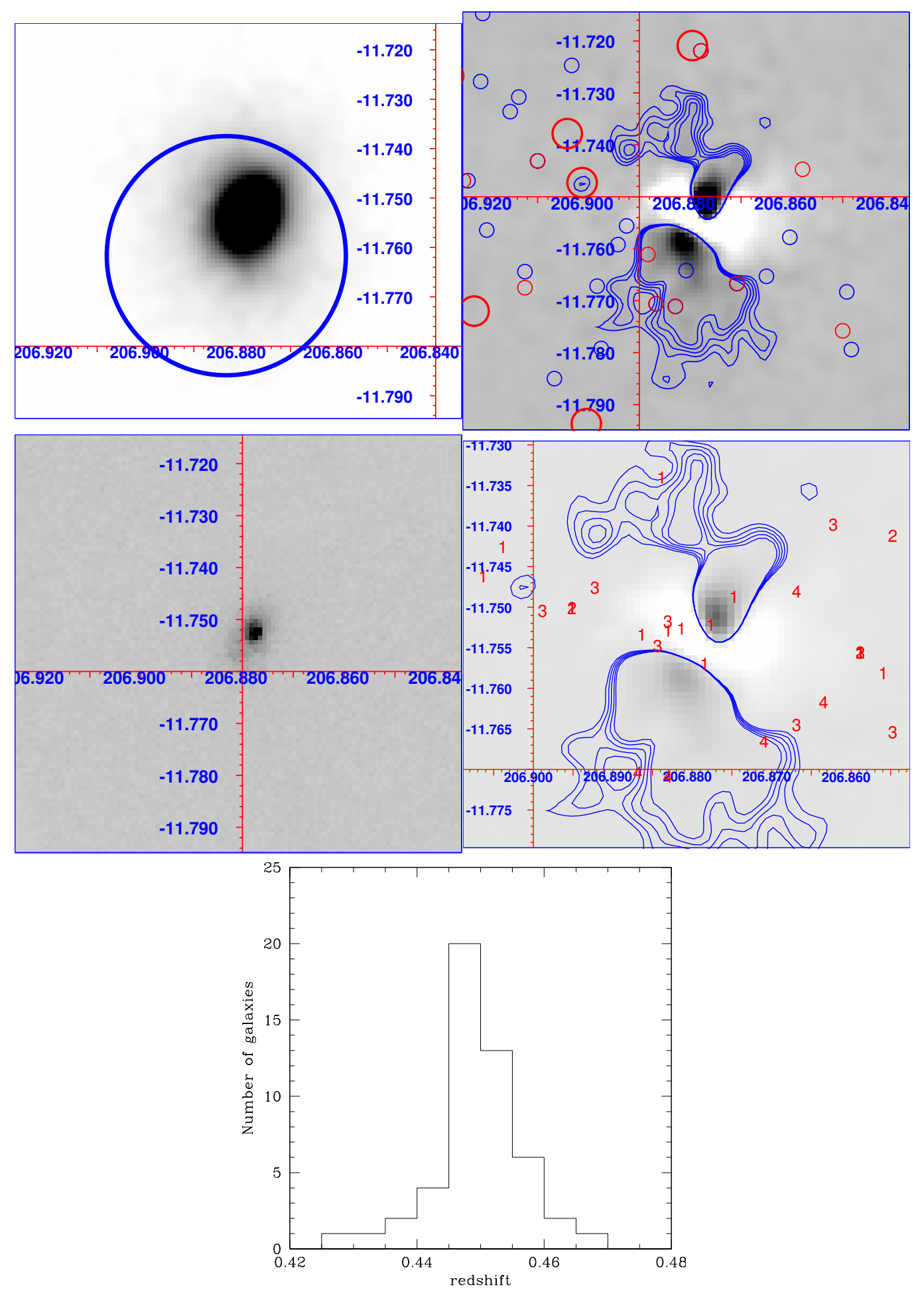

Fig. A.22. Same as Fig. A.2 for LCDCS 0829. 
L. Guennou et al.: Substructures in $0.4<z<0.9$ clusters
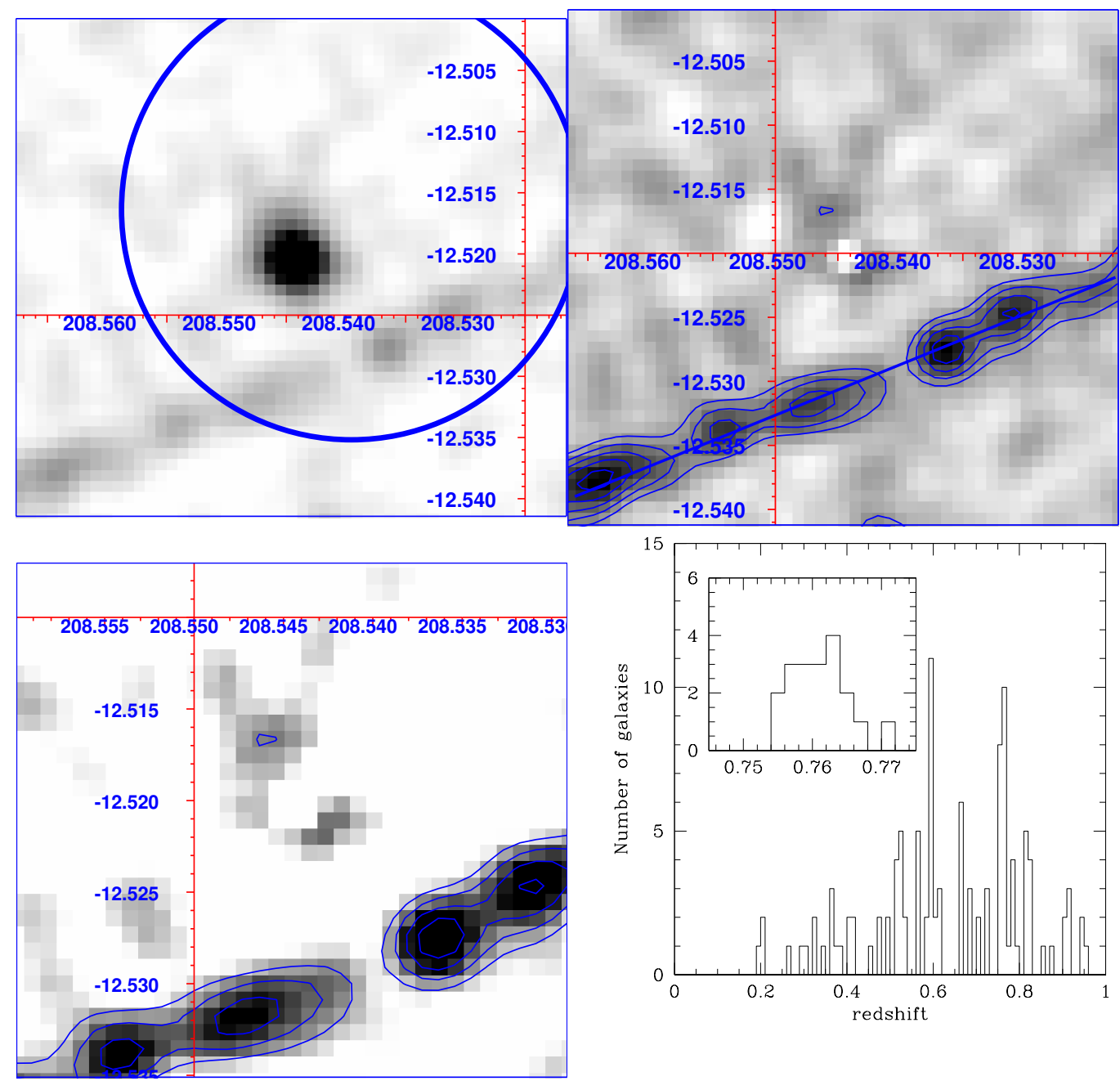

Fig. A.23. Same as Fig. A.1 for LCDCS 0853. 
A\&A 561, A112 (2014)

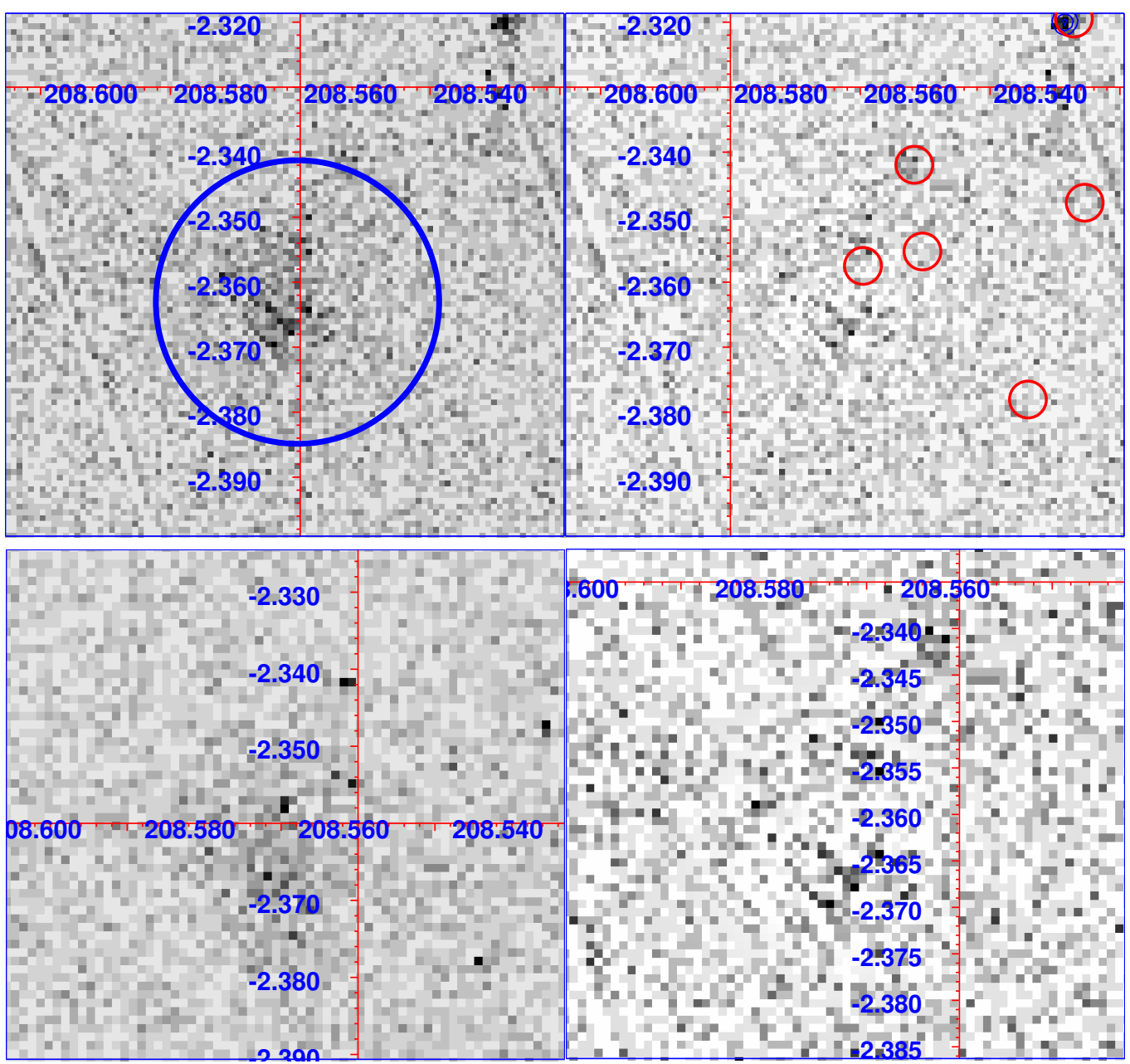

Fig. A.24. Same as Fig. A.7 for RX J1354.2-0221 
L. Guennou et al.: Substructures in $0.4<z<0.9$ clusters



Fig. A.25. Same as Fig. A.1 for MACS J1423.8+2404. We only used Chandra X-ray data for this cluster. 
A\&A 561, A112 (2014)
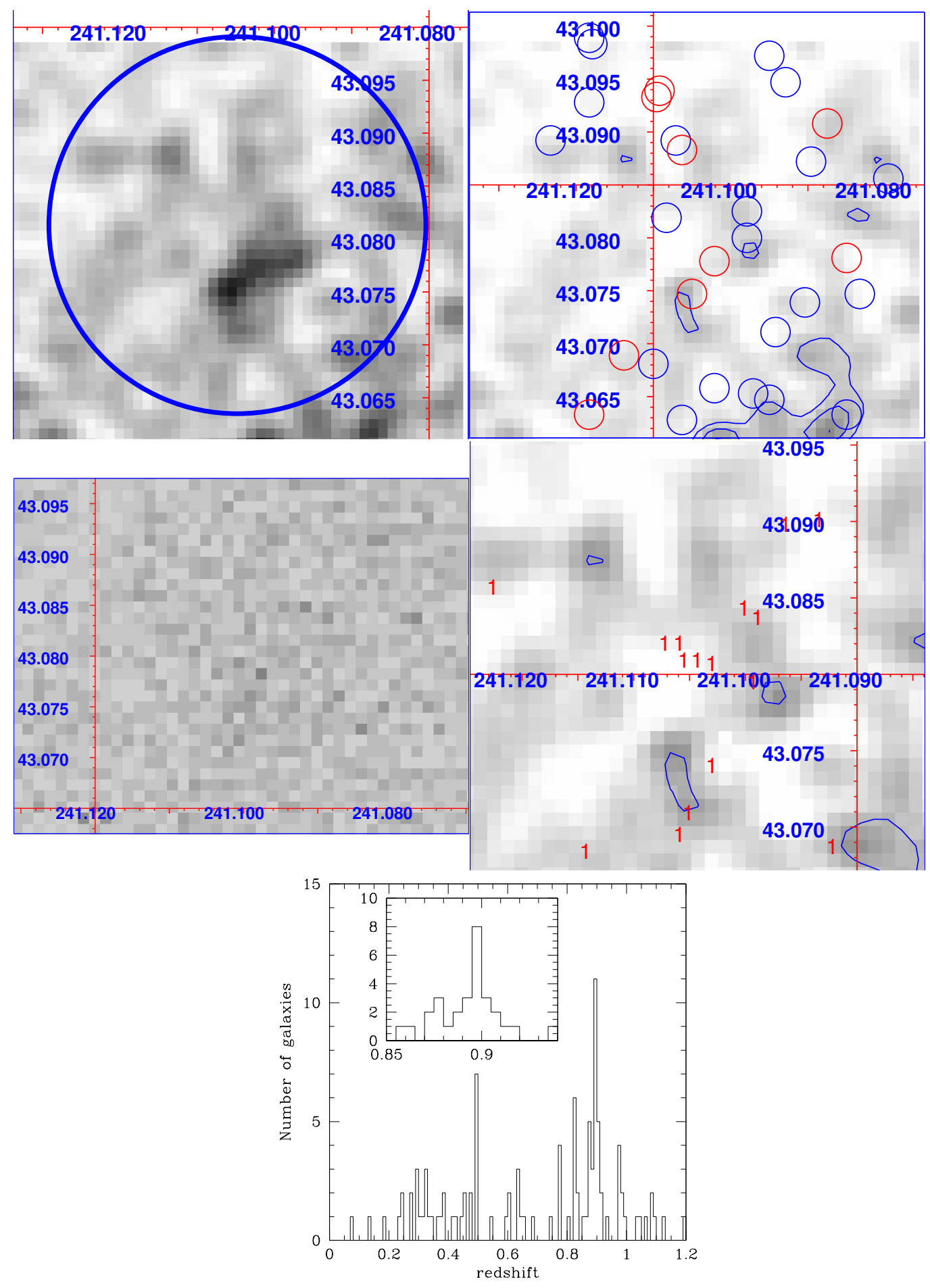

Fig. A.26. Same as Fig. A.2 for GHO 1602+4312. 
L. Guennou et al.: Substructures in $0.4<z<0.9$ clusters


Fig. A.27. Same as Fig. A.2 for MS 1621.5+2640. 
A\&A 561, A112 (2014)



Fig. A.28. Same as Fig. A.2 for MS 2053.7-0449. 
L. Guennou et al.: Substructures in $0.4<z<0.9$ clusters

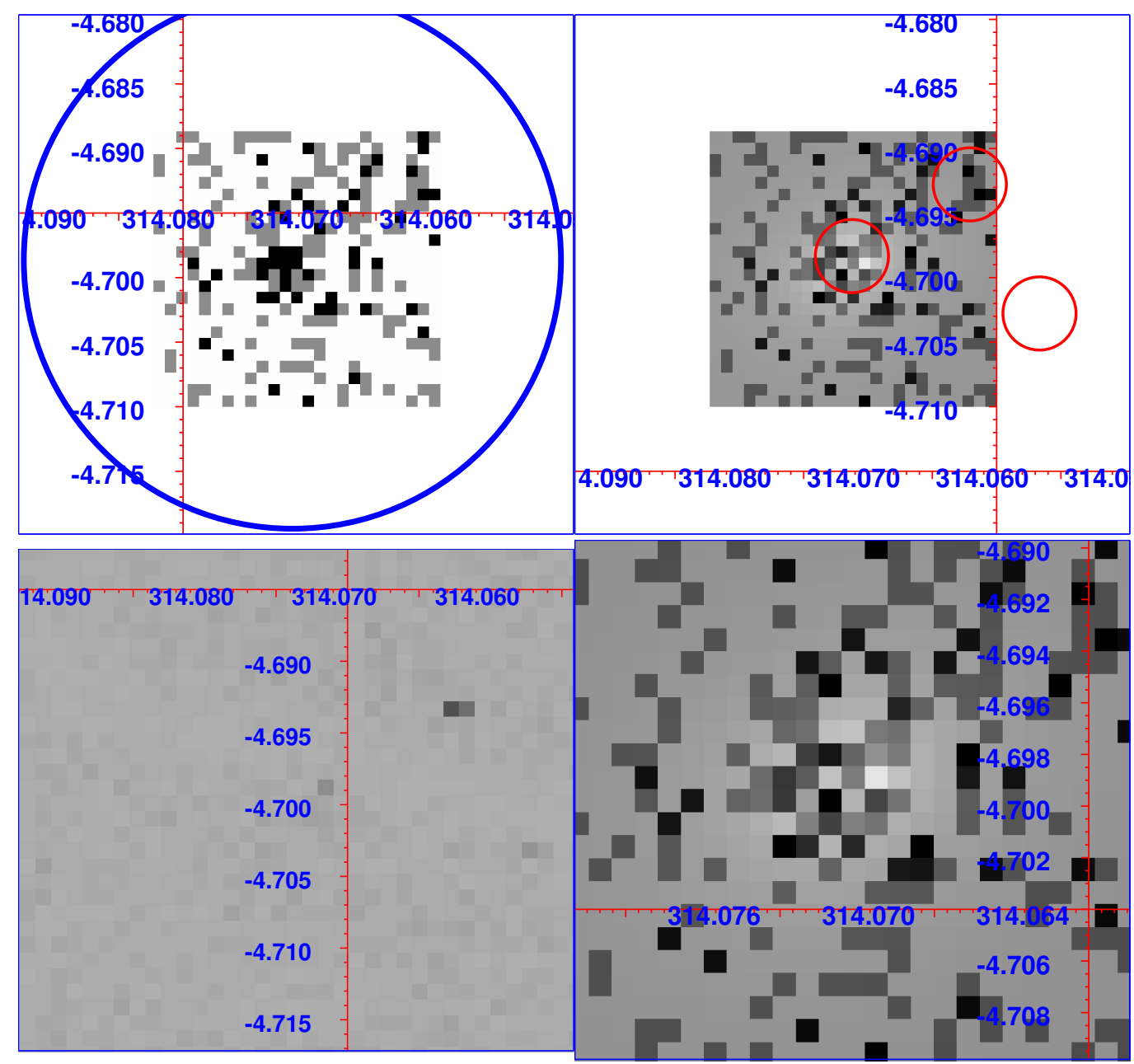

Fig. A.29. Same as Fig. A.7 for CXOSEXSI J205617.1-044155. 




Fig. A.30. Same as Fig. A.1 for GHO 2143+0408. 
L. Guennou et al.: Substructures in $0.4<z<0.9$ clusters



Fig. A.31. Same as Fig. A.3 for RX J2202.7-1902. 
A\&A 561, A112 (2014)
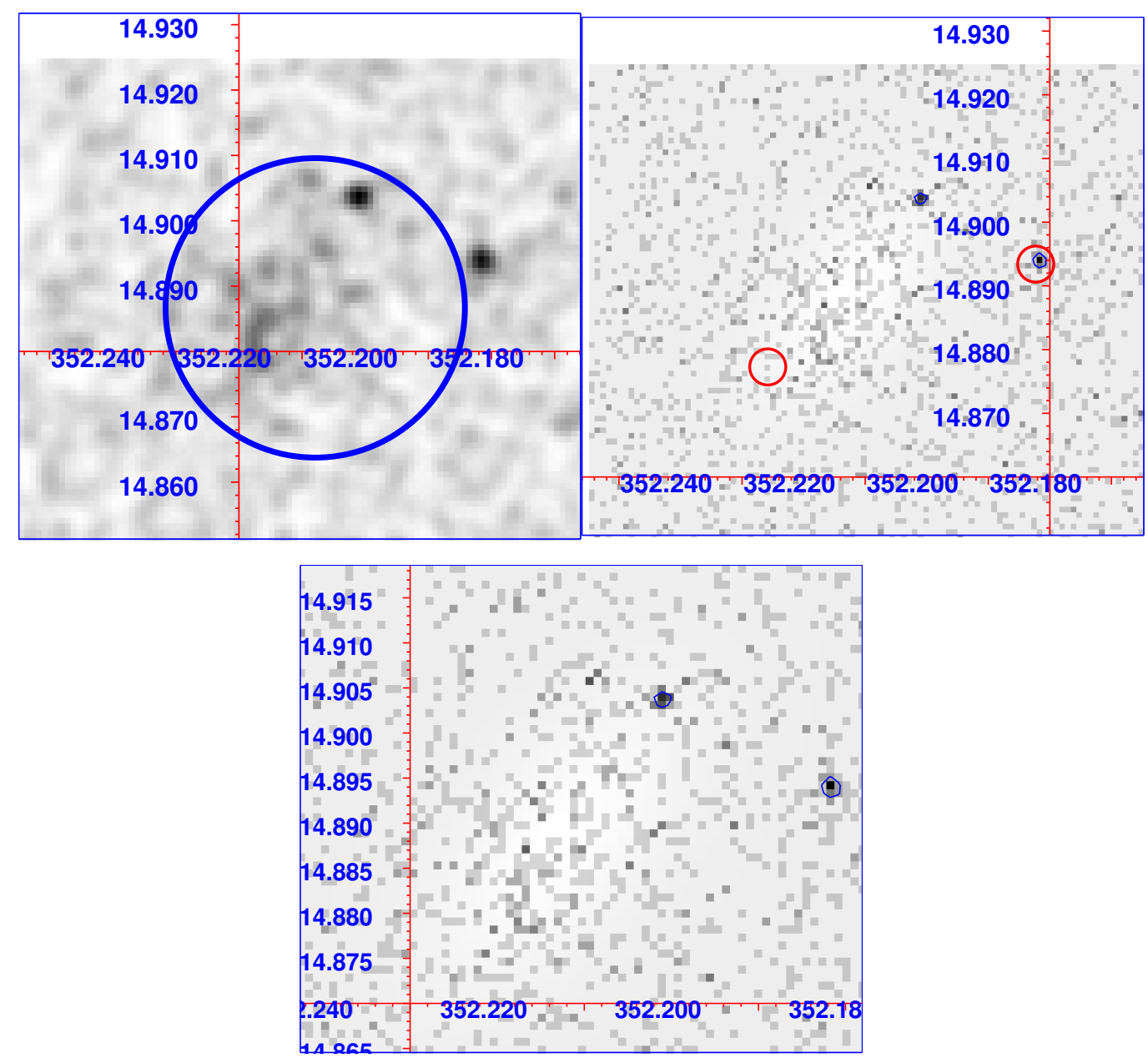

Fig. A.32. Same as Fig. A.3 for RX J2328.8+1453. 


\section{Appendix B: SG analysis for clusters with no usable XMM-Newton data}

We present in Sect. B the results of the SG analysis for the clusters with no usable XMM-Newton data, but with more than 15 redshifts in the cluster range.

\section{B.1. $\mathrm{Cl} J 0023+0423 B\left(5.96587^{\circ},+4.3780^{\circ}, z=0.8453\right)$}

There are 23 galaxies in the $[0.82,0.855]$ redshift range (Fig. B.1), and the SG method detects a single structure. We note the presence of several other structures on the line of sight at redshifts that are not very different from that of the cluster, suggesting we may be observing a filament seen close to face-on.

\section{B.2. CXOMP J002650.2+171935 (6.70917,$+17.3266^{\circ}$, $z=0.4907)$}

CXOMP J002650.2+171935 was discovered by Barkhouse et al. (2006) in the CHaMP survey. Its redshift histogram shows several small foreground structures and a background structure at $z \sim 0.5$. The main structure, corresponding to the cluster, has 29 galaxies in the [0.388, 0.402] range (Fig. B.2), and its redshift distribution is roughly symmetric.

The SG analysis detects a single structure.

\section{B.3. CXOMP J091126.6+055012 $\left(137.86083^{\circ},+5.8368^{\circ}\right.$, $z=0.7682)$}

CXOMP J091126.6+055012 was also discovered by Barkhouse et al. (2006) in the CHaMP survey. The XMM-Newton image of CXOMP J091126.6+055012 shows a very faint source at the position given by NED, too faint to be properly analysed, so we are including it in this section.

There is a peak in the redshift histogram at $z \sim 0.769$ (see Fig. B.3), with 18 galaxies with redshifts in the [0.758, 0.778] range. The SG analysis separates two structures within the main structure of the velocity histogram, and a third structure at redshift $z \sim 0.775$ (see Table 4). SG3 tends to be located north-east of the cluster centre.

\section{B.4. LCDCS $0110\left(159.46917^{\circ},-12.7289^{\circ}, z=0.5789\right)$}

The full redshift histogram shows a peak at $z=0.5789$ (the redshift given by NED for this cluster), and a larger peak at $z \sim 0.42$ (Fig. B.4). A weak lensing mass reconstruction shows the presence of two clusters close to each other on the line of sight (Clowe et al. 2006).

There are 18 galaxies in the $[0.575,0.585]$ redshift range. The SG method detects two main structures (see Table 4), with redshifts roughly corresponding to the two peaks of the zoomed redshift histogram. SG1 is east of the cluster centre and SG2 is west of the cluster centre.

\section{B.5. LCDCS $0130\left(160.17333^{\circ},-11.9308^{\circ}, z=0.7043\right)$}

The full redshift histogram shows several peaks, in particular two in front of and one behind the cluster, implying that this line of sight is intercepting various galaxy structures (Fig. B.5). There are 30 galaxies in the $[0.699,0.71]$ redshift range, where the redshift histogram is clearly asymmetric. The SG method detects a main structure and two less massive ones (see Table 4), in

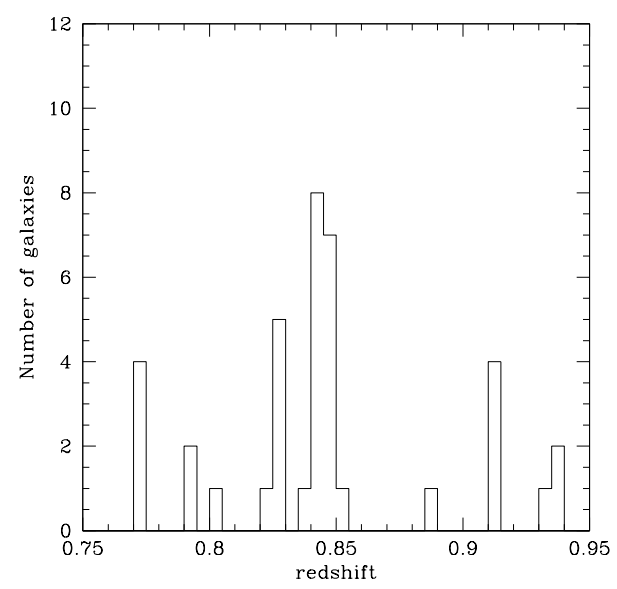

Fig. B.1. Redshift histogram for Cl J0023+0423B.

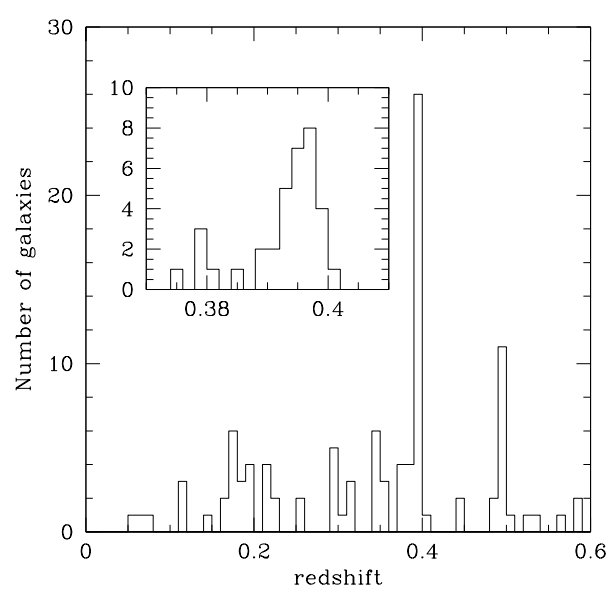

Fig. B.2. Redshift histogram for CXOMP J002650.2+171935. The insert shows a zoom around the cluster redshift.

agreement with possible substructuring found by Halliday et al. (2004).

\section{B.6. $\operatorname{LCDCS} 0172\left(\left(163.60083^{\circ},-11.7717^{\circ}, z=0.6972\right)\right.$}

The redshift histogram of LCDCS 0172 shows a prominent peak at $z \sim 0.697$ (Fig. B.6), with 48 galaxies in the [0.688, 0.705] range. There are also several smaller peaks, one in the foreground and two or three in the background of the cluster. The SG method finds three structures in the cluster (see Table 4).

\section{B.7. LCDCS $0173\left(163.68125^{\circ},-12.76389^{\circ}, z=0.7498\right)$}

The redshift histogram LCDCS 0173 shows a prominent peak at $z \sim 0.744$ (Fig. B.7), with 37 galaxies in the [0.741, 0.760] range. The SG method finds four structures with comparable masses (Table 4). A smaller peak is detected at $z \sim 0.73$, as also noted by Halliday et al. (2004).

\section{B.8. CL J1103.7-1245a $\left(165.89542^{\circ},-12.7794^{\circ}, z=0.6300\right)$}

Though available, the XMM-Newton image of CL J1103.7$1245 \mathrm{a}$ is too faint to be usable.

The full redshift histogram shows a peak at redshift 0.6300 (the cluster redshift given by NED) but another peak is also observed at $z \sim 0.72$, and this line of sight seems to be 

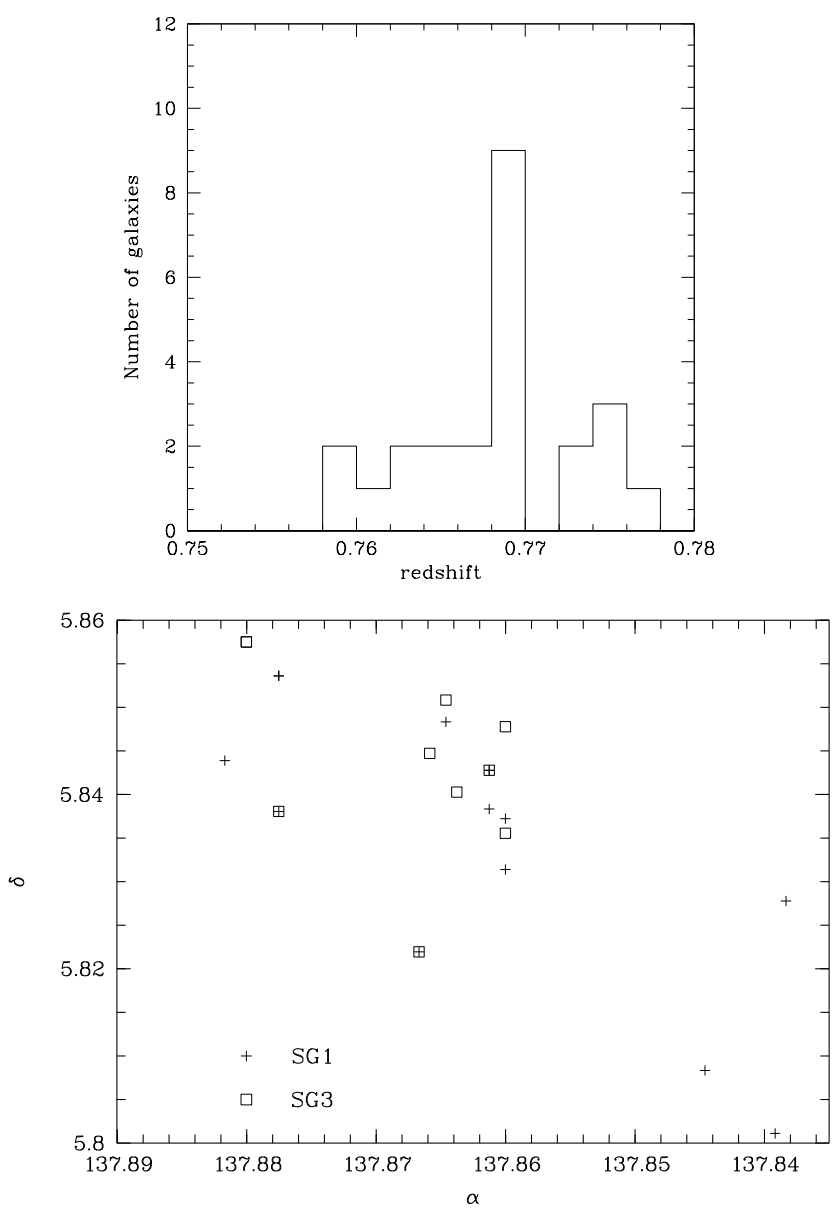

Fig. B.3. Upper panel: redshift histogram for CXOMP J091126.6+055012. Lower panel: SG1 and SG3 galaxy spatial distribution.

intersecting several galaxy structures (Fig. B.8), as already seen by Milvang-Jensen (2008). There are 19 galaxies with measured redshifts in the $[0.58,0.63]$ range, and the SG analysis finds a single massive structure.

\section{B.9. CXOMP J111726.1+074335 (169.358875,$+7.7264^{\circ}$, $z=0.4770)$}

The redshift histogram of CXOMP J111726.1+074335 (discovered by Barkhouse et al. 2006) shows a strong peak at the value given by NED: $z \sim 0.477$ (Fig. B.9). There are 36 galaxies in the $[0.476,0.500]$ range, with a clearly asymmetric distribution. The SG method finds two substructures of comparable masses (Table 4).

\section{B.10. LCDCS $0340\left(174.54292^{\circ},-11.5664^{\circ}, z=0.4798\right)$}

The redshift histogram of LCDCS 0340 shows a strong peak at $z \sim 0.48$ (Fig. B.10), with 51 galaxies in the [0.47, 0.49] range. The SG method finds six substructures with quite comparable masses (Table 4). Note that a foreground structure of 16 galaxies is detected in the $[0.4500,0.4585]$ redshift range, suggesting the presence of a second smaller cluster on the line of sight, as already noted by Milvang-Jensen et al. (2008).


Fig. B.4. Upper panel: redshift histogram for LCDCS 0110. The insert shows a zoom around the cluster redshift. Lower panel: SG1 and SG2 galaxy spatial distribution.

\section{B.11. $\operatorname{LCDCS} 0531\left(186.97458^{\circ},-11.6389^{\circ}, z=0.6355\right)$}

The XMM-Newton image is too faint to be usable.

There are 24 galaxies in the [0.63, 0.65] redshift range, but though the redshift histogram appears double-peaked (Fig. B.11), the SG method detects a single massive structure, in agreement with the lack of substructuring found by Clowe et al. (2006). Several smaller structures are detected on the line of sight.

\section{B.12. LCDCS $0541\left(188.12708^{\circ},-12.8425^{\circ}, z=0.5414\right)$}

The redshift histogram of LCDCS 0541 shows a strong peak at $z \sim 0.54$, with 80 galaxies in the [0.53, 0.555] range (Fig. B.12).

The SG analysis shows the existence of eight substructures of comparable mass, suggesting that this cluster is far from relaxed (Table 4). Based on a weak lensing analysis, Clowe et al. (2006) detected at least two mass peaks. X-ray data on this cluster would be very interesting for relating the optical substructures with the distribution of the X-ray gas.

\section{B.13. CIG J1236+6215 $\left(189.16500^{\circ},+62.2650^{\circ}, z=0.8500\right)$}

ClG J1236+6215 was serendipitously detected by Dawson et al. (2001) in the Hubble Deep Field North. The redshift histogram in the direction of this cluster shows a strong peak at $z \sim 0.85$, as well as many smaller peaks along the line of sight, including a 

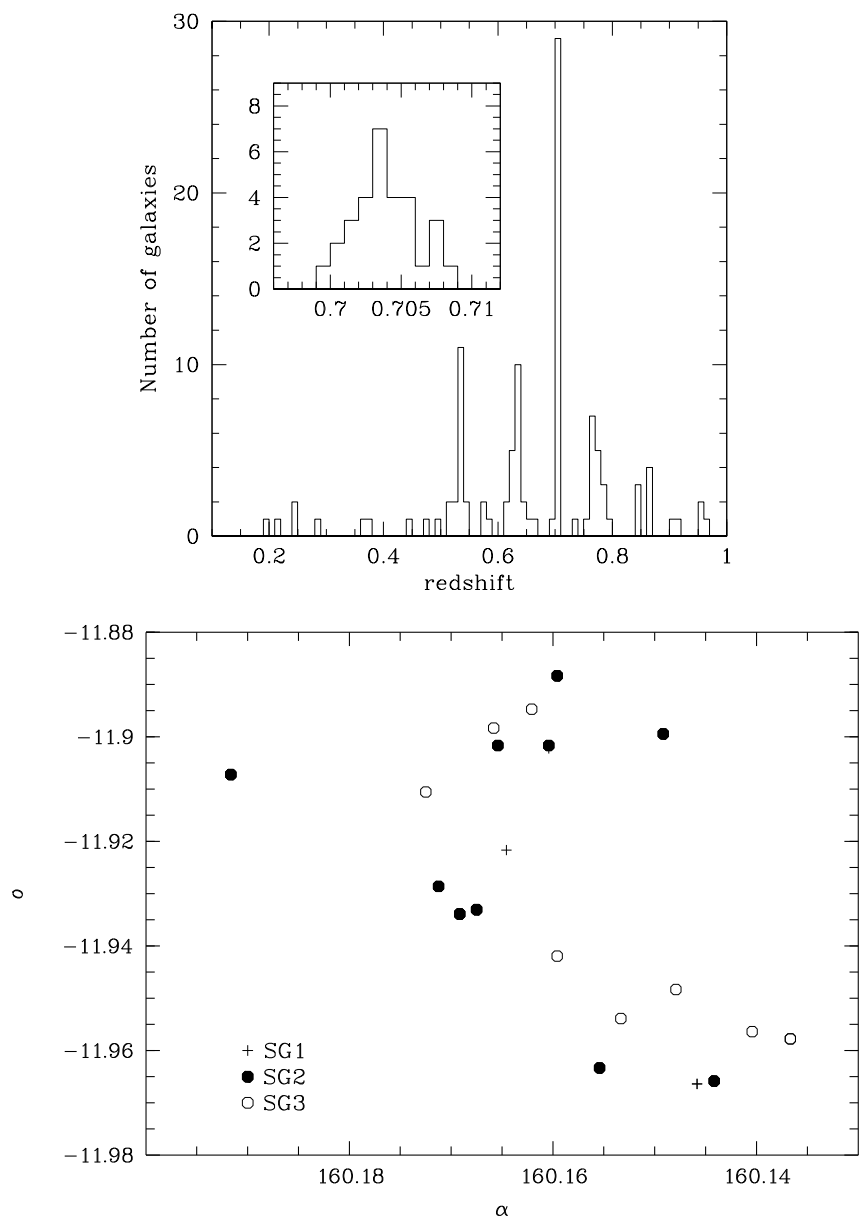

Fig. B.5. Upper panel: redshift histogram for LCDCS 0130. The insert shows a zoom around the cluster redshift. Lower panel: SG1, SG2, and SG3 galaxy spatial distribution.

foreground peak at $z \sim 0.53$ (Fig. B.13). There are 40 galaxies in the $[0.842,0.855]$ cluster range. The SG method finds four main substructures.

\section{B.14. XDCS mf J131001.9+322110 (197.50792,$+32.3528^{\circ}$, $z=0.4370)$}

XDCS mf J131001.9+322110 was discovered by Vikhlinin et al. (1998) but its XMM-Newton image is too faint to be usable.

The redshift histogram of XDCS mf J131001.9+322110 shows a strong, somewhat asymmetric, peak at $z \sim 0.293$. There are 19 galaxies in the [0.287, 0.300] cluster range (Fig. B.14), and the SG analysis finds a single structure.

\section{B.15. NSCS J132336+302223 $\left(200.91500^{\circ},+30.3760^{\circ}\right.$, $z=0.5080)$}

NSCS J132336+302223 was discovered by Gladders \& Yee (2005). Its redshift histogram shows a peak at $z \sim 0.46$, with 19 galaxies in the $[0.450,0.473]$ range (Fig. B.15). Since the number of galaxies is not very large and the redshift peak is broad, it is not straightforward to define the cluster redshift range exactly. We note, however, that the mean redshift $(z \sim 0.461)$ differs from the value $z=0.5080$ given by NED, at which we detect no peak in the redshift histogram. The SG analysis finds a single structure.
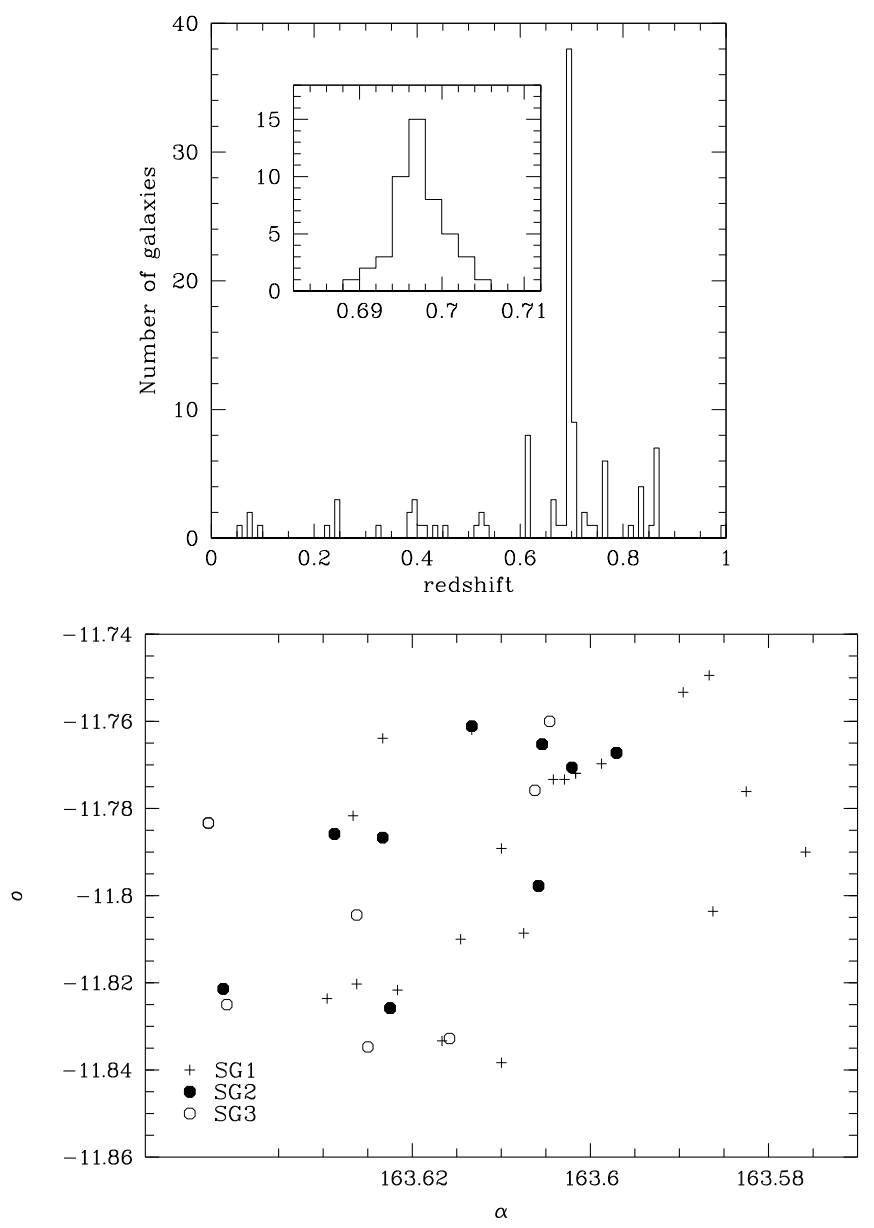

Fig. B.6. Upper panel: redshift histogram for LCDCS 0172. The insert shows a zoom around the cluster redshift. Lower panel: SG1, SG2, and SG3 galaxy spatial distribution.

\section{B.16. [MJM98] $034\left(203.80742^{\circ},+37.8156^{\circ}, z=0.5950\right)$}

[MJM98] 034 was first identified in a ROSAT field by McHardy et al. (1998). Its redshift histogram shows a peak at $z \sim 0.383$, with 16 galaxies in the $[0.380,0.386]$ range (Fig. B.16). Here again, the number of galaxies is not very large, and there is a small foreground structure of four galaxies at $z \sim 0.367$, which may (or may not) be related to the main cluster, so it is not straightforward to define the cluster redshift range exactly. Here also, the mean redshift $(z \sim 0.383)$ differs notably from the one given by NED (0.5950), where we detect no peak in the redshift histogram. The SG analysis finds a single structure.

\section{B.17. 3 C 295 Cluster $\left(212.83396^{\circ},+52.2025^{\circ}, z=0.4600\right)$}

The redshift histogram of the 3C 295 cluster shows a strong and rather broad peak at $z \sim 0.46$ (Fig. B.17), with 66 galaxies in the $[0.43,0.49]$ range. The cluster redshift range is probably narrower but in view of the redshift histogram alone, it is difficult to estimate it robustly. We can note, however, that the redshift histogram shows two peaks, and this is confirmed by the SG method, which finds two substructures of comparable masses (see Table 4), with velocities differing by only about $1000 \mathrm{~km} \mathrm{~s}^{-1}$.

We note that NED gives a redshift $z=0.2317$ for the $3 \mathrm{C} 295$ cluster and $z=0.4641$ for the 3C 295 radio galaxy, which appears rather confusing. In view of the large number of galaxies at 

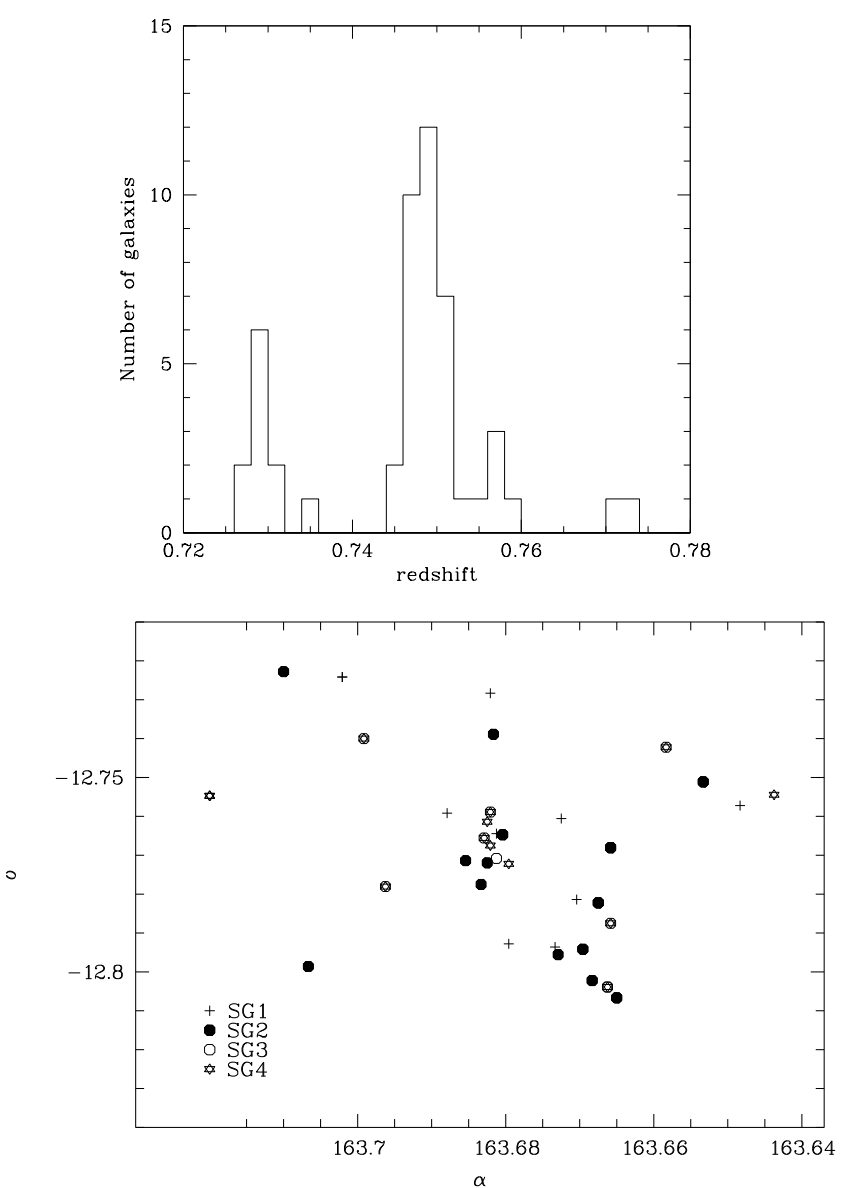

Fig. B.7. Upper panel: redshift histogram for LCDCS 0173. Lower panel: SG1, SG2, SG3, and SG4 galaxy spatial distribution.

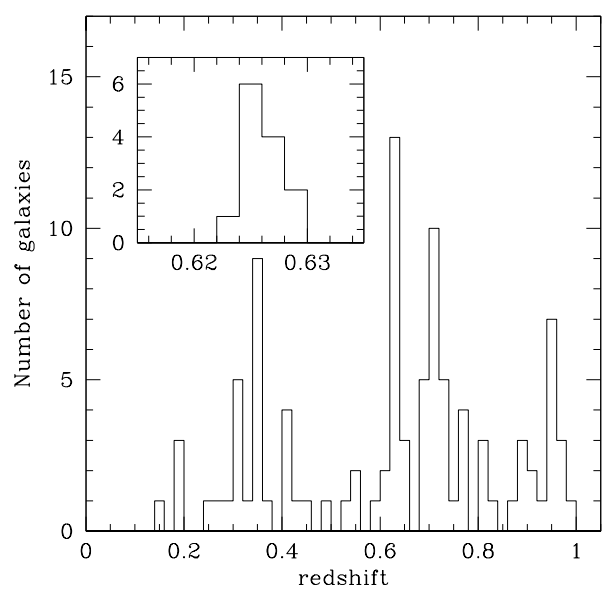

Fig. B.8. Redshift histogram for CL J1103.7-1245a. The insert shows a zoom around the cluster redshift.

redshift $\sim 0.46$, the cluster lies most probably at this redshift and not at $z=0.2317$, unless we are intercepting a filament along the line of sight.

B.18. GHO $1601+4253\left(240.80762^{\circ},+42.7601^{\circ}, z=0.5391\right)$

GHO 1601+4253 was discovered by Gunn et al. (1986). Its redshift histogram shows a strong and somewhat asymmetric peak at $z \sim 0.54$ (Fig. B.18). There are 50 galaxies in the [0.534,
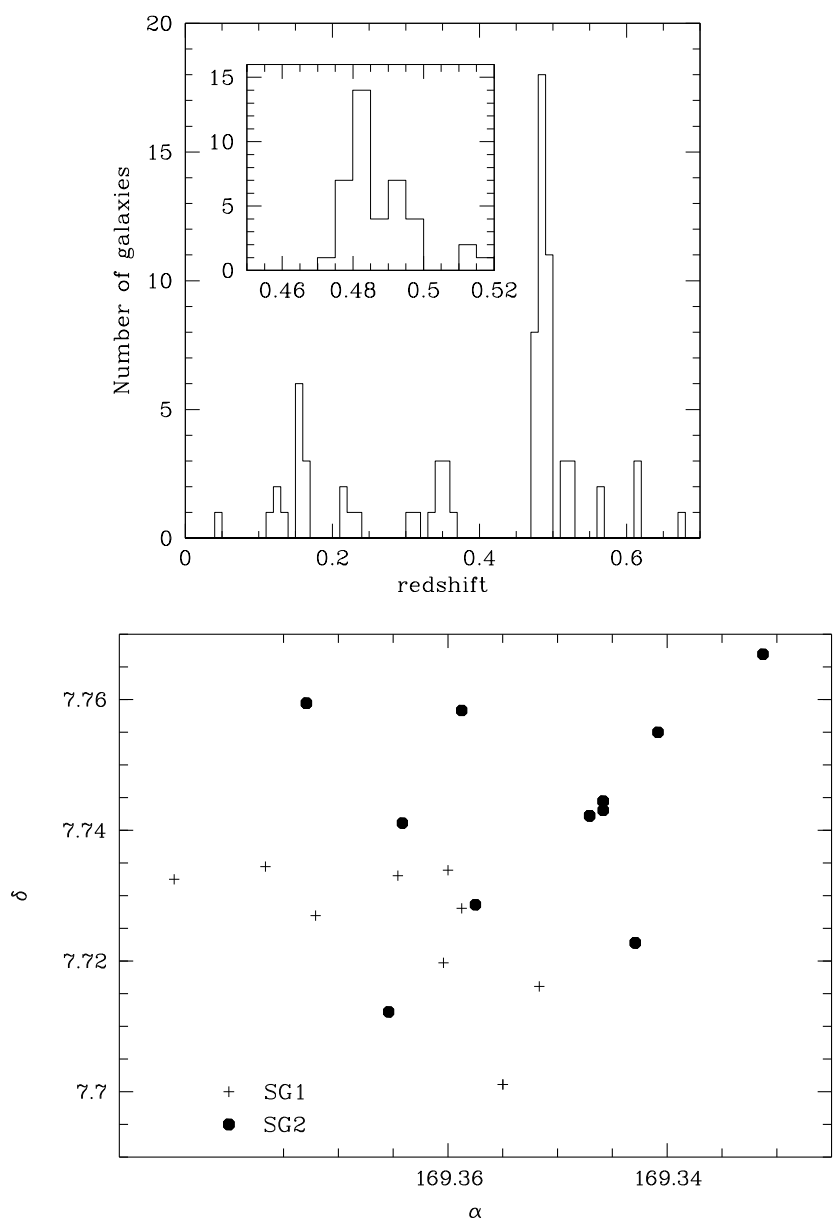

Fig. B.9. Lower panel: SG1 and SG2 galaxy spatial distribution. Upper panel: redshift histogram for CXOMP J111726.1+074335.

0.548] cluster range, and the SG method detects two substructures (see Table 4).

\section{B.19. $R X J 1716.4+6708\left(259.20667^{\circ},+67.1417^{\circ}\right.$, $z=0.8130)$}

RX J1716.4+6708 was discovered by Henry et al. (1997), who gave a redshift $z=0.8130$ for this cluster and measured 12 galaxy redshifts in the [0.7945, 0.8266] range, spreading along a filament. Gioia et al. (1999) increased this sample and published a list of 37 galaxies with spectroscopic redshifts in the $[0.7924,0.8256]$ range. The velocity dispersion calculated from these 37 redshifts is rather large: $1522 \mathrm{~km} \mathrm{~s}^{-1}$ and agrees with the suggestion by Henry et al. (1997) that RX J1716.4+6708 is instead a protocluster still in the process of forming. This was confirmed by the weak lensing analysis of Clowe et al. (1998), who stated that RX J1716.4+6708 is not a well-formed cluster. The SG analysis finds a large structure (the cluster) and a few small substructures too poorly sampled to be characterized.

The XMM-Newton image of this cluster is too faint to be usable. 
L. Guennou et al.: Substructures in $0.4<z<0.9$ clusters


Fig. B.10. Upper panel: redshift histogram for LCDCS 0340. The insert shows a zoom around the cluster redshift. Lower panel: SG1, SG2, SG3, SG4, SG5, and SG6 galaxy spatial distribution.



Fig. B.11. Redshift histogram for LCDCS 0531. The insert shows a zoom around the cluster redshift.


Fig. B.12. Upper panel: redshift histogram for LCDCS 0541. Lower panel: SG1, SG2, SG3, SG4, SG5, SG6, SG7, and SG8 galaxy spatial distribution. 
A\&A 561, A112 (2014)


Fig. B.13. Upper panel: redshift histogram for ClG J1236+6215. The insert shows a zoom around the cluster redshift. Lower panel: SG1, SG2, SG3, and SG4 galaxy spatial distribution.



Fig. B.14. Redshift histogram for XDCS mf J131001.9+322110.



Fig. B.15. Redshift histogram for NSCS J132336+302223.

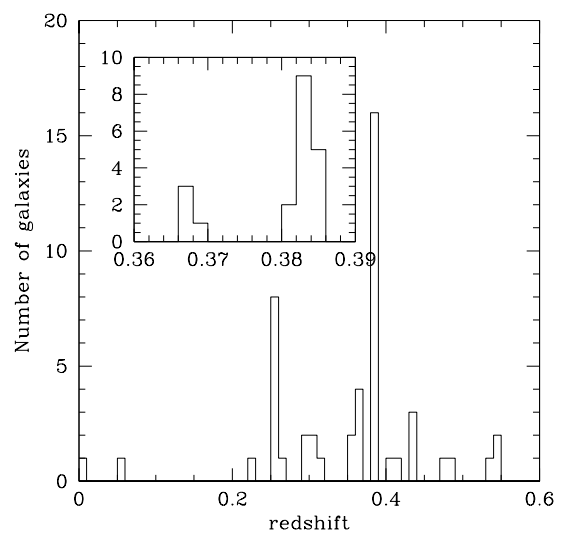

Fig. B.16. Redshift histogram for [MJM98] 034. 
L. Guennou et al.: Substructures in $0.4<z<0.9$ clusters
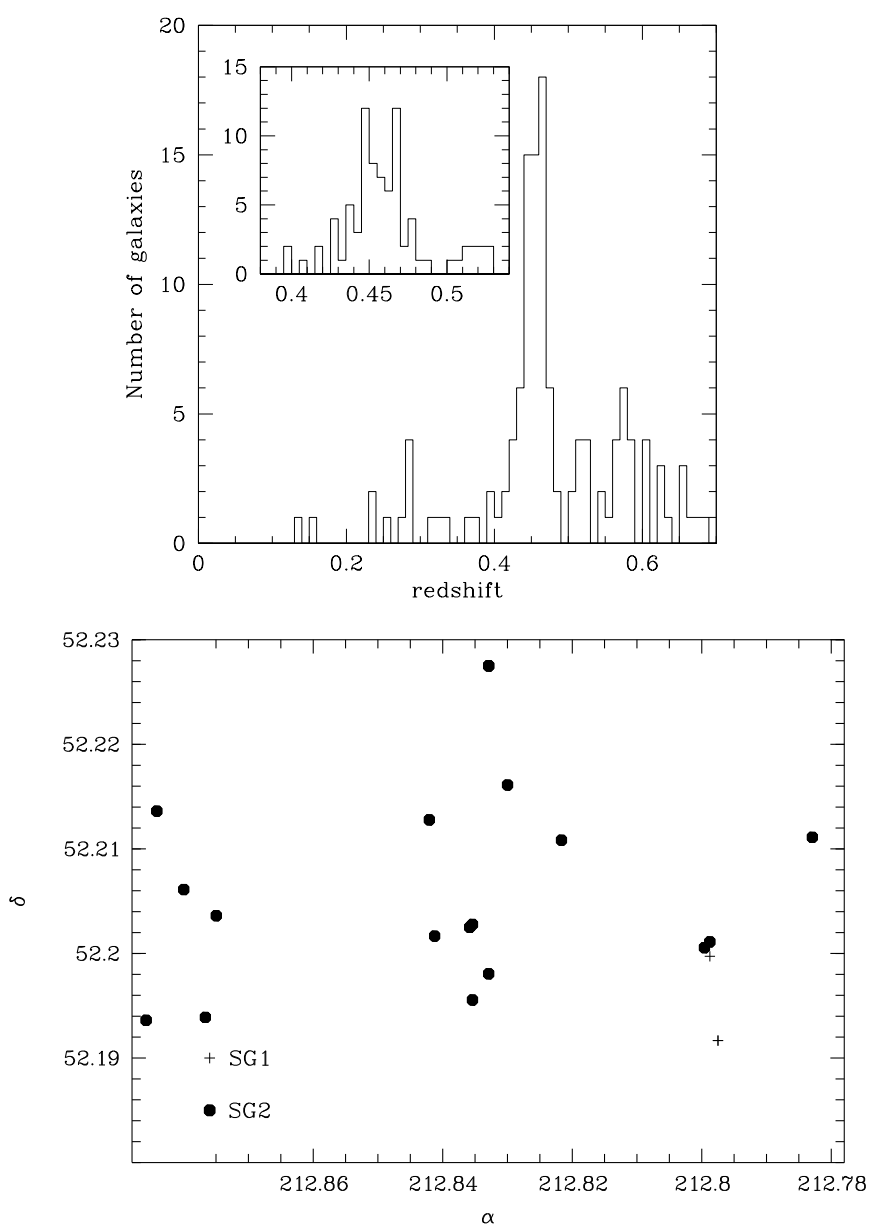

Fig. B.17. Upper panel: redshift histogram for Cluster 3C 295. Lower panel: SG1 and SG2 galaxy spatial distribution.
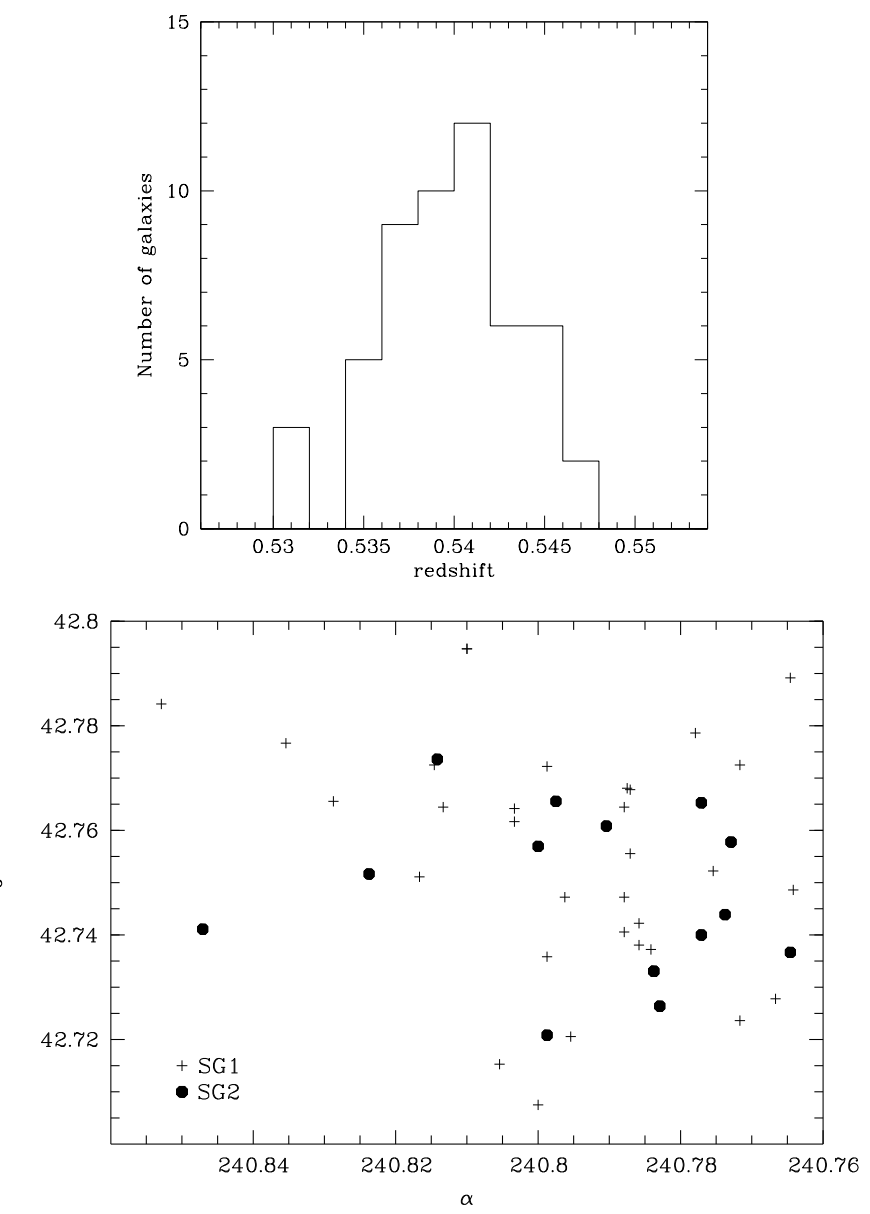

Fig. B.18. Upper panel: redshift histogram for GHO 1601+4253. Lower panel: SG1 and SG2 galaxy spatial distribution.

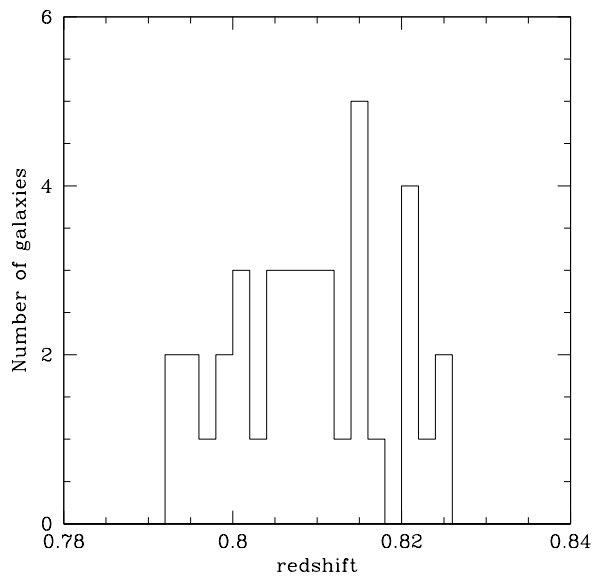

Fig. B.19. Redshift histogram for RX J1716.4+6708. 


\section{Appendix C: Notes on clusters with no usable XMM-Newton data and no possible SG analysis}

We give below a few notes on clusters with no usable XMM-Newton data (see Table C.1) and too few galaxy redshifts for an SG analysis, but they may be of some interest for various reasons, principally because in a number of cases the redshift does not correspond to what is given by NED.

\section{C.1. Abell $2843\left(14.15573^{\circ},-27.5130^{\circ}, z=0.215 / 0.5600 ?\right)$}

Little is known on Abell 2843 (Abell et al. 1989). According to NED and Simbad, its redshift is 0.5600 , but the redshift histogram shows no galaxy at this redshift (Fig. C.1). On the other hand, a peak is detected at $z \sim 0.215$, with 11 galaxies in the $[0.21,0.22]$ redshift range.

\section{C.2. $R X J 0848.8+4455\left(132.20542^{\circ},+44.9294^{\circ}\right.$, $z=0.5430 ?)$}

According to Holden et al. (2001), there are two objects in this zone: RX J0848+4456, an X-ray emitting cluster of galaxies at a redshift $z=0.570$, and a group at a slightly lower redshift, $z=0.543$. These authors state that the lower redshift group has, at most, one-fifth and, more likely, one-tenth, of the X-ray luminosity of RX J0848+4456.

The redshift histogram in the direction of this cluster only shows a small peak in the region of the cluster redshift given by $\operatorname{NED}(z=0.5430)$ (Fig. C.2), with 6 galaxies in the [0.54, 0.57] range. The redshift histogram shows a large peak at redshift at $z \sim 1.26$, due to the existence of a background cluster in the Lynx field (Holden et al. 2001).

\section{C.3. $\mathrm{GHO} 0940+4819\left(145.92105^{\circ},+48.0873^{\circ}, z=0.4700 ?\right)$}

This cluster was first identified by Gunn et al. (1986). NED gives four different redshifts for the same cluster at the same coordinates, so the redshift of this cluster remains uncertain.

\section{C.4. $R X J 0957.8+6534\left(149.47167^{\circ},+65.5750^{\circ}\right.$, $z=0.5300 ?)$}

There are only three galaxies in NED around this cluster position, and only one at the cluster redshift $(z=0.5300)$.

\section{C.5. SEXCLAS $12\left(163.15917^{\circ},+57.5137^{\circ}, z=0.6100 ?\right)$}

SEXCLAS 12 is not significantly detected with XMM-Newton following our criteria.

This cluster and the following one (they are about 3 arcmin apart) were taken from the sample of Kolokotronis et al. (2006), who used XMM-Newton pointings and five-band optical data to serendipitously identify X-ray selected clusters.

The full redshift histogram of SEXCLAS 12 shows no prominent peak, in particular at the redshift $z=0.6100$ given by NED (Fig. C.3). Four galaxies at $z \sim 0.64$ could indicate that this is the cluster redshift, but more spectra are obviously needed to securely assign a redshift to these two potential structures.



Fig. C.1. Redshift histogram for Abell 2843. The insert shows a zoom around the possible alternative cluster redshift.



Fig. C.2. Redshift histogram for RX J0848.8+4455.

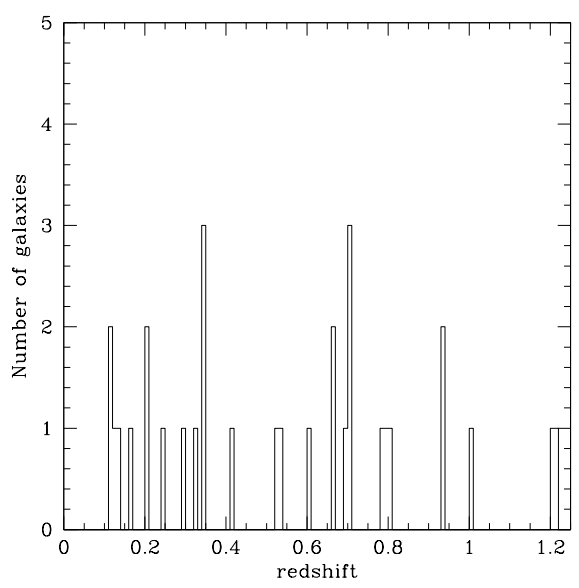

Fig. C.3. Redshift histogram for SEXCLAS 12.

\section{C.6. SEXCLAS $13\left(163.22583^{\circ},+57.5360^{\circ}, z=0.5800\right)$}

SEXCLAS 13 is very close to SEXCLAS 12, and is not detected with XMM-Newton either with our criteria.

The full redshift histogram of SEXCLAS 13 shows no prominent peak either, in particular at the redshift $z=0.5800$ given by NED, where there are only three galaxies (Fig. C.3). 
Table C.1. Clusters with no usable XMM-Newton data.

\begin{tabular}{lcccc}
\hline \hline Name & RA & Dec & $z$ & $N z$ \\
\hline Abell 2843 & 14.15573 & -27.51298 & $0.215(0.560)$ & 11 \\
RX J0848.8+4455 & 132.20542 & 44.92944 & 0.5430 & 6 \\
GHO 0940+4819 & 145.92105 & 48.08725 & $0.4700 ?$ & 4 \\
RX J0957.8+6534 & 149.47167 & 65.57500 & 0.5300 & 1 \\
SEXCLASS 12 & 163.15917 & 57.51369 & $0.64(0.6100)$ & 4 \\
SEXCLASS 13 & 163.22583 & 57.53600 & 0.5800 & 3 \\
RX J1540.8+1445 & 235.22208 & 14.75944 & 0.442 & 11 \\
CL J1604+4314 & 241.10750 & 43.23972 & $0.925(0.8652)$ & 11 \\
OC02 J1701+6412 & 255.34583 & 64.23583 & 0.4518 & 1 \\
\hline
\end{tabular}

Notes. (1) Name (as in NED), (2) right ascension in degrees (J2000.0), (3) declination in degrees (J2000.0), (4) redshift (in parentheses, redshift given by NED, if different), (5) approximate number of galaxies with redshifts in the cluster range.

Table C.2. New spectroscopic redshift measurements obtained with VLT/FORS2 for galaxies located in the RX J1540.8+1445 and GHO $2143+0408$ clusters.

\begin{tabular}{|c|c|c|c|c|c|}
\hline \multicolumn{3}{|c|}{ RX J1540.8+1445 } & \multicolumn{3}{|c|}{ GHO $2143+0408$} \\
\hline RA & Dec & $z$ & RA & Dec & $z$ \\
\hline 235.1656134 & 14.7352182 & 0.4427 & 326.4801 & 4.4216 & 0.5562 \\
\hline 235.1680243 & 14.8065969 & 0.4699 & 326.4843 & 4.4264 & 0.5259 \\
\hline 235.1693535 & 14.7595151 & 0.19192 & 326.4862 & 4.3711 & 0.7385 \\
\hline 235.1727369 & 14.8025443 & 0.5621 & 326.487 & 4.4145 & 0.4237 \\
\hline 235.1751678 & 14.7894211 & 1.0626 & 326.4872 & 4.4158 & 0.0 \\
\hline 235.1784827 & 14.7920345 & 0.411 & 326.4904 & 4.4179 & 0.1736 \\
\hline 235.1815337 & 14.8060684 & 0.4418 & 326.4945 & 4.3659 & 0.4029 \\
\hline 235.1896165 & 14.752223 & 0.4415 & 326.4949 & 4.3676 & 0.4015 \\
\hline 235.1899269 & 14.7878709 & 0.0 & 326.4963 & 4.413 & 0.657 \\
\hline 235.1969677 & 14.77182 & 0.19516 & 326.5008 & 4.4087 & 0.5171 \\
\hline 235.1989185 & 14.7371372 & 0.8428 & 326.5008 & 4.4087 & 0.5171 \\
\hline 235.1991002 & 14.7479399 & 0.6009 & 326.5058 & 4.4166 & 0.6034 \\
\hline 235.2003501 & 14.7968349 & 0.4423 & 326.508 & 4.41 & 0.0 \\
\hline 235.2007918 & 14.7198888 & 0.4407 & 326.5084 & 4.351 & 0.235 \\
\hline 235.2034364 & 14.7284524 & 0.4444 & 326.5103 & 4.4317 & 0.601 \\
\hline 235.204883 & 14.7792275 & 0.5638 & 326.5126 & 4.3905 & 0.5251 \\
\hline 235.206242 & 14.7412534 & 0.442 & 326.5131 & 4.4235 & 0.5584 \\
\hline 235.2062595 & 14.7961091 & 0.605 & 326.5136 & 4.3634 & 0.809 \\
\hline 235.2103902 & 14.7983381 & 0.19469 & 326.5152 & 4.3999 & 0.6641 \\
\hline 235.2158803 & 14.7617361 & 0.7473 & 326.5197 & 4.3671 & 0.0 \\
\hline 235.2181524 & 14.7638333 & 0.1925 & 326.5198 & 4.3587 & 0.6479 \\
\hline 235.2191101 & 14.7743322 & 0.4428 & 326.5211 & 4.3734 & 0.5137 \\
\hline 235.2191998 & 14.7723376 & 0.4346 & 326.5215 & 4.3511 & 0.595 \\
\hline 235.2217389 & 14.7770748 & 0.4419 & 326.5217 & 4.3566 & 0.78 \\
\hline 235.2244809 & 14.7584889 & 0.5703 & 326.5234 & 4.4028 & 0.9993 \\
\hline 235.2246155 & 14.7657406 & 0.44033 & 326.5235 & 4.376 & 0.0 \\
\hline 235.227457 & 14.7573185 & 0.7356 & 326.5256 & 4.3886 & 0.6517 \\
\hline 235.2332704 & 14.7323585 & 0.7659 & 326.527 & 4.3871 & 0.0374 \\
\hline 235.2406444 & 14.7849472 & 1.9096 & 326.5425 & 4.3626 & 1.0079 \\
\hline 235.24072 & 14.7824836 & 0.2831 & & & \\
\hline 235.2611534 & 14.7588384 & 0.0 & & & \\
\hline
\end{tabular}

Notes. The columns are: right ascension, declination, and redshift.

$$
\begin{aligned}
& \text { C.7. } R X J 1540.8+1445\left(235.22208^{\circ},+14.7594^{\circ},\right. \\
& z=0.4410)
\end{aligned}
$$

Little is known about RX J1540.8+1445, which was discovered in X-rays by Vikhlinin et al. (1998). We have obtained spectroscopic redshifts for galaxies in this cluster with VLT/FORS2 (see Table C.2). However, only 11 redshifts are in the $[0.43,0.45]$ interval (see Fig. C.5) so we cannot apply an SG.

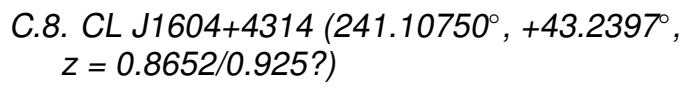

CL J1604+4314 is part of a supercluster made of several clusters at redshift $\sim 0.9$ analysed in detail by Gal \& Lubin (2004), and by Gal et al. (2005, 2008), with several hundred spectroscopically confirmed members in a very large spatial area. Unfortunately, these redshifts are not publicly available, so we cannot give a redshift histogram or apply the SG method to this cluster. NED 




Fig. C.4. Redshift histogram for SEXCLAS 13.

gives $z=0.8652$ but the peak in the 11 redshifts available in NED rather indicates $z \sim 0.925$ (based on 11 galaxies), in agreement with Gal \& Lubin (2004).

\section{C.9. OC02 J1701+6412 (255.34583 ${ }^{\circ},+64.2358^{\circ}$ $z=0.4518 ?)$}

OC02 J1701+6412 was discovered by Vikhlinin et al. (1998) in their ROSAT PSPC galaxy cluster survey, where they give for this cluster a redshift $z=0.4530$ (the redshift of the $\mathrm{cD}$ galaxy). Out of the 88 redshifts gathered in an area of 5 arcmin radius around this cluster there is only one galaxy at $z=0.4518$, most the other redshifts being distant galaxies (Fig. C.6). There are in particular several galaxies at $z \sim 0.22$ and a large peak at $z \sim 2.3$ (17 galaxies with redshifts in the $[2.28,2.31]$ redshift range).

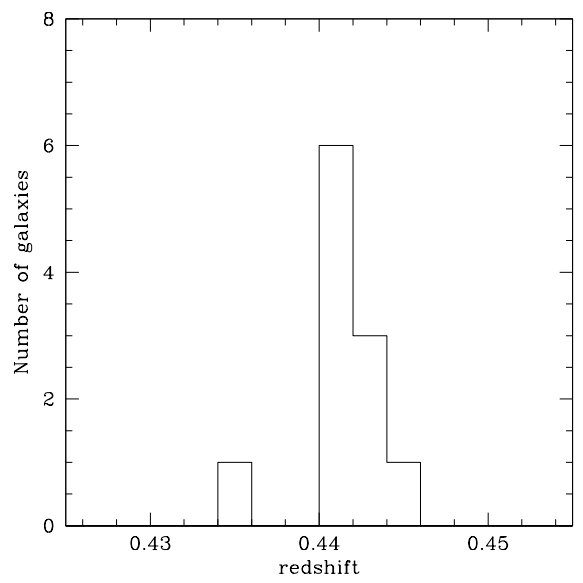

Fig. C.5. Redshift histogram for RX J1540.8+1445.

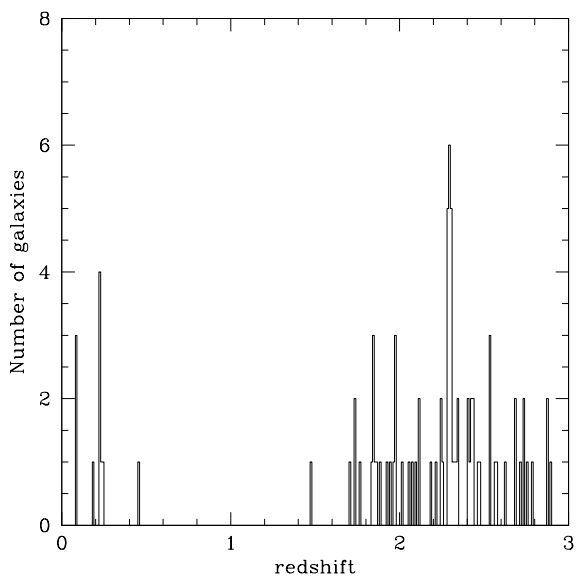

Fig. C.6. Redshift histogram for OC02 J1701+6412. 